DESY-THESIS-1998-025

August 1998

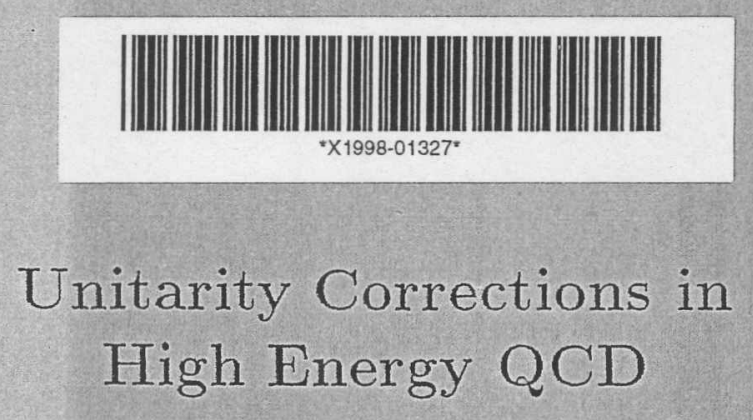

by

C. Ewerz 
DESY behält sich alle Rechte für den Fall der Schutzrechtserteilung und für die wirtschaftliche Verwertung der in diesem Bericht enthaltenen Informationen vor.

DESY reserves all rights for commercial use of information included in this report, especially in case of filing application for or grant of patents.

To be sure that your reports and preprints are promptly included in the HEP literature database send them to (if possible by air mail):

\begin{tabular}{|l|l|}
\hline DESY & DESY \\
Zentralbibliothek & Bibliothek \\
Notkestraße 85 & Platanenallee 6 \\
22603 Hamburg & 15738 Zeuthen \\
Germany & Germany \\
\hline
\end{tabular}




\title{
Unitarity Corrections in High Energy QCD
}

\author{
Dissertation \\ zur Erlangung des Doktorgrades \\ des Fachbereichs Physik \\ der Universität Hamburg
}

vorgelegt von

Carlo Ewerz

aus Köln

Hamburg

1998 
Gutachter der Dissertation:

Gutachter der Disputation:

Datum der Disputation:

Sprecher des Fachbereichs Physik und Vorsitzender des Promotionsausschusses:
Prof. Dr. J. Bartels

Prof. Dr. W. Buchmüller

Prof. Dr. J. Bartels

Prof. Dr. G. Kramer

30. Juni 1998

Prof. Dr. B. Kramer 


\begin{abstract}
The subject of this thesis is the Regge limit of Quantum Chromodynamics. The scattering of virtual photons at very large center of mass energy is studied in the framework of perturbation theory. It is known that the approximation which resums leading logarithms in the energy (the BFKL Pomeron) violates unitarity at asymptotically large energies. The BFKL Pomeron describes the exchange of two interacting reggeized gluons in the $t$-channel. We investigate unitarity corrections to the BFKL Pomeron which are characterized by a larger number of gluons in the $t$-channel. They are described by $n$-gluon amplitudes which obey a tower of coupled integral equations. We review the known results on the threeand four-gluon amplitudes. These results indicate that the unitarity corrections can be cast into a $2+1$-dimensional conformal field theory. It is the aim of this thesis to gain further insight into this field theory structure by investigating higher $n$-gluon amplitudes. We develop methods which allow to proceed to higher $n$-gluon amplitudes, and we study the five- and six-gluon amplitudes. We pay special attention to the mechanism leading to the reggeization of the gluon and to the field theory structure of the unitarity corrections. The integral equation for the five-gluon amplitude is solved. Our investigation of the mechanism of reggeization results in Ward type identities for the amplitudes. In addition we find the color tensors describing the reggeization of the gluon in higher orders. The first steps in the investigation of the six-gluon amplitude are performed, and further evidence for a field theory structure of the unitarity corrections is found.
\end{abstract}

\title{
Zusammenfassung
}

Gegenstand dieser Arbeit ist der Regge-Limes der Quantenchromodynamik. Im Rahmen der Störungstheorie wird die Streuung von virtuellen Photonen bei sehr hoher Schwerpunktsenergie untersucht. Die Resummation führender Logarithmen in der Energie führt zu einer Näherung (dem BFKL Pomeron), die bei asymptotisch großen Energien Unitarität verletzt. Das BFKL Pomeron beschreibt den Austausch von zwei wechselwirkenden reggeierten Gluonen im $t$-Kanal. Wir untersuchen Unitaritätskorrekturen zum BFKL Pomeron, die durch eine größere Anzahl von Gluonen im $t$-Kanal ausgezeichnet sind. Sie werden beschrieben durch $n$-Gluonamplituden, die einen Satz von gekoppelten Integralgleichungen erfüllen. Wir geben eine Zusammenfassung der bekannten Resultate über die 3- und 4-Gluonamplituden. Diese Resultate legen nahe, daß die Unitaritätskorrekturen durch eine 2+1-dimensionale Feldtheorie beschrieben werden können. Ziel der vorliegenden Arbeit ist es, durch die Untersuchung höherer $n$-Gluonamplituden weitere Einsicht in diese feldtheoretische Struktur zu gewinnen. Wir entwickeln Methoden, die die Untersuchung höherer $n$-Gluonamplituden zulassen, und analysieren die 5und 6-Gluonamplituden. Die Gleichung für die 5-Gluonamplitude wird gelöst. Unsere besondere Aufmerksamkeit richtet sich auf den Mechanismus der Reggeierung des Gluons. Wir finden Ward-artige Identitäten für die Amplituden, die den Mechanismus der Reggeierung näher beleuchten. Außerdem finden wir Farbtensoren, die die Reggeierung des Gluons in höheren Ordnungen beschreiben. Die ersten Schritte in der Untersuchung der 6-Gluonamplitude werden unternommen. Es ergeben sich weitere deutliche Hinweise auf eine zugrundeliegende feldtheoretische Struktur der Unitaritätskorrekturen. 


\section{Contents}

Introduction 1

1 BFKL Equation and Violation of Unitarity 7

1.1 The BFKL equation . . . . . . . . . . . . . 7

1.2 Violation of unitarity . . . . . . . . . . . . 10

1.3 Reggeization of the gluon . . . . . . . . . . . . . . 10

1.4 Conformal invariance in impact parameter space . . . . . . . . . . 11

2 Integral Equations for $n$-gluon Amplitudes 13

2.1 The $n$-gluon amplitudes . . . . . . . . . . . . . . . . 13

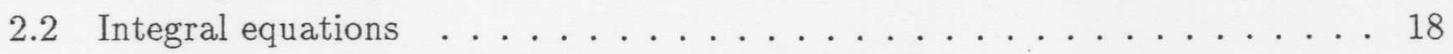

2.3 Color structure . . . . . . . . . . . . . . . . . . . . . . . . 21

2.4 The quark loop . . . . . . . . . . . . . . . . . . . 24

2.5 Reggeon momentum diagrams . . . . . . . . . . . . . . . 28

2.6 Integral kernels . . . . . . . . . . . . . . . . . . . . . 29

2.7 Standard integrals . . . . . . . . . . . . . 30

3 Three and Four Gluons, the Transition Vertex $V_{2 \rightarrow 4} \quad 33$

3.1 The three-gluon amplitude. . . . . . . . . . . . . 33

3.2 The four-gluon amplitude and the two-to-four transition vertex $V_{2 \rightarrow 4} \ldots 34$

3.3 Field theory structure . . . . . . . . . . . . . . 37

3.4 Conformal invariance in impact parameter space . . . . . . . . . . . 39

3.5 New representation for the vertex $V_{2 \rightarrow 4} \ldots \ldots \ldots \ldots \ldots \ldots$

4 Five Gluons 44

4.1 A reggeizing part and the integral equation for the remaining part . . . . . 44

4.2 Solving the equation for the remaining part . . . . . . . . . . . . 46

4.3 Interpretation of the result $\ldots \ldots \ldots \ldots$. . . . . . 50

5 Ward Type Identities and Reggeization Tensors 52

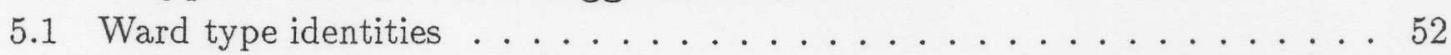

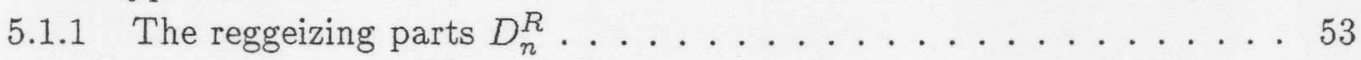

5.1 .2 The amplitudes $D_{4}^{I}$ and $D_{5}^{I} \ldots \ldots \ldots \ldots \ldots$

5.1 .3 Significance of the Ward type identities . . . . . . . . . . . 57

5.2 Reggeization tensors in color space . . . . . . . . . . . . . 58 
6 Six Gluons 65

6.1 A reggeizing part . . . . . . . . . . . . . . . . . 65

6.2 The integral equation for the remaining part . . . . . . . . . . 66

6.3 A new piece in the field theory . . . . . . . . . . . . . . . 69

6.3 .1 Explicit representation and properties ............ . . 69

6.3.2 Interpretation in view of an effective field theory . . . . . . . 72

6.4 Further reggeization . . . . . . . . . . . . . . . . . . . 72

6.5 On a possible Pomeron-Odderon-Odderon vertex . . . . . . . . . . . 77

6.6 The six-gluon amplitude and the effective field theory . . . . . . . . . 78

$\begin{array}{lr}\text { Summary and Outlook } & 80\end{array}$

A Color Algebra 83

A.1 A method for contractions in $\operatorname{su}\left(N_{c}\right)$ algebra . . . . . . . . . 83

A.2 Useful contractions of color tensors . . . . . . . . . . . . . 85

B A Combinatorial Method for the Momentum Space Integrals $\quad 88$

$\begin{array}{lr}\text { Bibliography } & 93\end{array}$ 


\section{Introduction}

Quantum Chromodynamics is established as the microscopic theory of strong interactions and has been successfully applied to a variety of phenomena over the past 25 years. But even after this long period of intensive research a number of deep problems in QCD remain to be solved. One of the major challenges is to understand the high energy behavior of $\mathrm{QCD}$ in the Regge limit. The focus of Regge physics is to explore hadron scattering at very large center of mass energies $s$ and fixed momentum transfer $t$ of the order of a hadronic mass scale. In pre-QCD times, Regge theory [1] was developed to describe hadronic scattering processes at high energies. Regge theory was based on the fundamental principles of analyticity, unitarity and Lorentz invariance. It was found that in the kinematic region of the Regge limit, the behavior of scattering amplitudes is determined by the singularities the Regge limit, the behavior of scattering amplitudes is determined by the singularities the so-called Regge poles and cuts. The optical theorem relates total cross sections to the imaginary part of the forward elastic scattering amplitude. This sparks the interest in Regge singularities contributing to elastic scattering, namely those associated with $t$-channel exchanges carrying vacuum quantum numbers. The high energy asymptotics of total cross sections receives its leading contribution from the rightmost singularity in

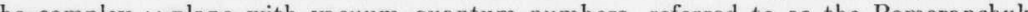
the complex $\omega$-plane

Starting from these concepts an impressive development in theoretical physics took place. Inspired by Regge theory very successful phenomenological models were built to describe high energy scattering. In 1967 Gribov invented a reggeon field theory [2] that soon evolved into a subject of intensive study [3]. Further, it was possible to relate Regge poles to the hadron spectrum by crossing symmetry which in turn is a consequence of analyticity. All known strongly interacting particles were discovered to be reggeons, that is to lie on remarkably straight Rerge trajectories passing through the physical spin at the squared mass of the squared mas of the particle belong to the deep tris in in to the background when Quantum Chonodynamics was discovered to be the correct gauge theory of strong interactions. With the advent of the Tevatron and the HERA machine the interest in the Regge limit of hadronic scattering amplitudes was revived. A specific model that very successfully describes total hadronic cross sections up to the highest available energies at the Tevatron is the so-called soft Pomeron model [4]. This model is especially simple and fits all available elastic scattering data by a function that slowly grows like a power with the center of mass energy $s$, the exponent being 0.08 .

To the present day a profound understanding of the Regge limit within the framework of $\mathrm{QCD}$ is missing. The ultimate goal would be to calculate the position of Regge poles and cuts in the $\omega$-plane from first principles. Due to the difficult mathematical structure 
of QCD this will not be possible for the foreseeable future. The main difficulty is that the kinematical region of the Rege limit is characterized by high parton densities and therefore of genuinely non-perturbative nature. Moreover, the wealth of hadronic scattering fore of genuinely non-perturbative nature. Moreover, the wealth of hadronic scattering
processes at very high energies is dominated by low momentum scales and perturbation processes at very high energies is dominated by low momentum scales and perturbation
theory cannot be applied. The current knowledge of the non-perturbative regime of QCD on the other hand is by far not sufficient to make unambiguous predictions for the Regge asymptotics.

Fortunately there is a class of scattering processes that allow a perturbative treatment even at high energies, namely hard scattering processes. They are distinguished by the deminates the whole process. The cross sections of such processes are of view. They make it possible to approach the dificult region of the Regge limit from a side where perturbation theory is reliable. The hope is of course that the transition is in a certain sense smooth. The best examples for such interesting processes are heavy onium scattering and the scattering of highly virtual photons in which the virtualities of the two photons should ideally be of comparable size. In the former process the hard scale is provided by the large mass of the quark and the antiquark. Whereas that process is of purely academic interest, the latter will be observable $[5,6]$ at the planned Next Linear Collider (NLC) facility. Another process of similar, but less inclusive kind is the production of hard forward jets in deep inelastic scattering, so-called Mueller jets [7]. They have been further investigated in $[8,9]$.

The hard scale ensures the smallness of the strong coupling constant $\alpha_{s}$. But its smallness can be compensated by large logarithms of the energy $s$, necessitating a resummation of contributions of the order $\alpha_{s} \log (s)$. This approximation scheme is called the leading logarithmic approximation (LLA). Lipatov and his coworkers applied it first to a massive gauge theory [10] and then to QCD [11]. The resulting resummed amplitude has a cut in the complex angular momentum plane. This singularity is the celebrated Balitskii-FadinKuraev-Lipatov (BFKL) or perturbative Pomeron. It arises as the solution of an integral equation that resums an infinite amount of Feynman diagrams contributing in LLA. This BFKL equation can be written as a Schrödinger equation for partial waves in transverse momentum space. Its energy-like variable is the complex angular momentum $\omega$, and its momentum space. bound state of two gluons. The amplitude has a ladder toplogy, the ladder rungs between the two gluons are effective interaction kernels. The gluons are reggeized gluons [12], eac of them represents an infinite sum of Feynman diagrams. The reggeized gluons have been identified as the appropriate degrees of freedom in high-energy QCD, and they can b regarded as collective excitations of the Yang-Mills field. A striking feature of the BFKL amplitude is its conformal invariance in impact parameter space. It was discovered in [13] and made it possible to solve the BFKL equation also for the non-forward direction.

It became clear immediately that the leading logarithmic approximation is in conflict with a profound principle of field theory, namely unitarity. The Froissart-Martin theorem $[14,15]$ is derived from unitarity and states that total hadronic cross sections cannot grow faster than logarithmic with energy. The total cross section of a scattering process in LLA, however, grows like a power with the center of mass energy $s$. The corresponding exponent as it results from the position of the leading singularity in the $\omega$-plane equals $\omega_{\mathrm{BFKL}}=\alpha_{s} N_{c} / \pi 4 \ln 2 \simeq 0.5\left(1+\omega_{\mathrm{BFKL}}\right.$ is called the Pomeron intercept $)$. Therefore the BFKL Pomeron eventually violates unitarity at asymptotically high energies. The leading logarithmic approximation is in this sense inconsistent. A full understanding of the Regge limit of QCD will certainly require a consistent framework, and the approximation scheme has to be extended beyond the leading logarithms.

The growth of cross sections at high energies is connected with the increasing parton densities inside the colliding hadrons that will inevitably lead to an overlapping of the partons. It is intuitively clear that parton cascades can then no longer be regarded as independent and recombination effects will become important. That will eventually lead to saturation, thereby taming the growth of the cross sections. The leading logarith mic approximation can be extended in a way that reflects this intuitive picture of unitarization. The resulting scheme is known as the generalized leading logarithmic approximation (GLLA). In this scheme a minimal set of non-leading corrections is identified that leads to a unitary scattering amplitude. The distinctive property of these unitarity corrections unitary scattering a ext bers of $t$-channel gluons. The first attempt to include such corrections was made in [16]. The most complete and systematic approach to unitarity corrections was formulated by Bartels $[17,18,19]$. It is ideally applied to the theoretically very clean process of highly virtual photon $\left(\gamma^{*} \gamma^{*}\right)$ scattering. With the help of $t$-channel unitarity relations one defines partial wave amplitudes in two-dimensional transverse momentum space describing the production of a given number $n$ of gluons in the $t$-channel. The BFKL amplitude naturally appears in this approach as the first approximation.

With the inclusion of contributions with more gluons in the $t$-channel a new question arises, namely that of $s$-channel unitarity in the subchannels. If one decides to demand unitarity only in the direct channel one has to take into account only contributions in which the number of gluons in the $t$-channel is fixed. The corresponding partial wave amplitudes obey the Bartels-Kwieciński-Praszalowicz (BKP) equations $[18,20]$. These are Schrödinger type equations similar to the BFKL equation, but now including all pairwise interactions of the $n$ gluons. If on the other hand one demands unitarity also in all possible subchannels, one is forced to take into account also contributions in which the number of gluons changes in the $t$-channel. The corresponding integral equations were derived in [19]. In addition to the pairwise interactions of the gluons they contain number-changing transition kernels. Due to this the different $n$-gluon amplitudes are connected and the integral equations are turned into a tower of coupled equations. It should be emphasized acter simplifying assumptions concerning the gauge group are made.

The inclusion of contributions in which the number of gluons in the $t$-channel is not conserved has highly non-trivial consequences and implies a very interesting structure. The three- and four-gluon amplitudes were first investigated by Bartels and Wüsthoff in $[21,22]$. It was observed that the amplitudes consist only of very few basic elements. The three-gluon amplitude was found to be a superposition of two-gluon states, as was a part of the four-gluon amplitude. The remaining part of the four-gluon amplitude involves a two-gluon state as well as an irreducible four-gluon compound state, described by the BFKL and the four-particle BKP equation respectively. They are coupled to each other by an efective two to four turns the quantum mechanical problen of the n-glaon compound states into a quantum be interpreted in the framework of an effective field theory of unitarity corrections. 
This observation gives rise to the hope that the whole set of unitarity corrections can be cast into the form of an effective field theory. From the results that have been obtained so far, some of its fundamental properties can already be inferred. The field theory is an field theory in two space-like dimensions and one time-like dimension. The latter is realized as rapidity. The elements of the field theory are Green functions describing the $t$-channel propagation of interacting $n$-gluon compound states, and number-changin vertices. Soon that it exhibis confornaling that it exhits corameter space [23]. That immediately leads to the conjecture that the unitarity corrections can even be described by a $2+1$ dimensional conformal field theory where the two space-like dimensions are now identified with impact parameter space instead of transverse momentum space. Further evidence for the interpretation of the unitarity corrections as a conformal field theory was found in [24] where the triple Pomeron vertex was found to have the form of a conformal three-point function. However, it is known that the conformal invariance is a property that does not persist when next-to-leading logarithmic corrections are taken into account. The runing coll of the coupling constant is bour worth the effort to study the symmetry, its breaking can then be investigated in a second step.

A prerequisite for the emergence of the field theory structure is the fact that parts of the amplitudes reduce to two-gluon amplitudes, i. e. have the analytic properties of superpositions of BFKL amplitudes. This phenomenon generalizes the well-known reggeization of the gluon and has therefore been termed reggeization as well. When the $n$-gluon amplitudes are used to reconstruct the full scattering amplitude via $t$-channel unitarity the reggeizing parts of the amplitudes in fact lead to higher order corrections to the Regge trajectory of the gluon.

The structure found in the unitarity corrections is very encouraging so far. But the knowledge is still rather limited since up to this point only the amplitudes with up to four $t$-channel gluons have been investigated. Only the simplest elements of the effective field theory are known, and the general underlying rules for their construction have not yet been understood. It is the main purpose of this thesis to extend our knowledge beyond the three- and four-gluon amplitudes and to learn more about the properties of the effective field theory of unitarity corrections. The natural next step is to investigate the five- and six-gluon amplitudes. Within the framework of the integral equations this even seems to be the only way to proceed. This is becane the equations are coupled and do not allow to find an amplitude will be especially interesting since we expect new elements to occur only in amplitudes with an even number of gluons. This is clearly suggested by the complete reggeization of the threegluon amplitude. We will pay special attention to reggeization since it is intimately related to the emergence of the field theory structure. The color structure of the amplitudes will be very important in this respect. We consider a very special scattering process, i. e. $\gamma^{*} \gamma^{*}$ scattering, and we will only be able to investigate the $n$-gluon amplitudes for a very limited range of $n$. The hope is of course that the information gained under the special conditions will be of more get natere. Before givg a me conditions will be of more general nature. Before giving a more detailed outline of this thesis, we briefly mention related results and complementary approaches to the problem of the Regge limit of QCD

In the recent past there has been impressive progress in the field of high energy QCD, especially in the perturbative approach. The next-to-leading logarithmic (NLLA) correc- tions to the BFKL equation have been found in an almost decade-lasting effort [25]. Parts of the calculation have also been performed indendently in [26]. The investigation of the consequences has just started $[27,28]$. The corrections to the intercept of the Pomeron turn out to be large, but the qualitative picture is arguably not altered. The large- $N_{c}$ limit of the BKP equations has been a field of very active research. Lipatov discovered that the $n$-gluon compound state in the large- $N_{c}$ limit is an integrable system [29]. Faddeev re quantum nverse scattering method and the Bethe ansatz, and very interesting results have been obtained [31]. A further recent result is the resolution of the longstanding problem of the Odderon in perturbative QCD. The Odderon is the $C=-1$ partner of the Pomeron, i. e. it carries negative charge parity. In perturbative $\mathrm{QCD}$ it is described by a completely symmetric three-gluon compound state that obeys the three-particle BKP equation. Its conformal invariance had been used in [32] to place constraints on its wavefunction. Janik and Wosiek recently calculated its intercept which turned out to be smaller than one [33]. Mueller formulated a very elegant approach to high energy QCD in which color dipoles play an important role [34]. This color dipole picture of high energy onium-onium scattering was shown to be equivalent to the BFKL Pomeron [35]. The dipole picture was also used was shown to be equivalent to the BFKL Pomeron [35]. The dipole picture was also used
to study the unitarity problem $[36,37,38]$. Other approaches are the formulation of an effective action for the Regge limit $[39,40]$, a similar approach also aiming at a simplified effective theory for high energy scattering [41], and the method of operator expansion [42]. Approaches of a more non-perturbative kind are the eikonal approximation in a soft gluon background [43], the model of the stochastic vacuum [44], and the semiclassical approach [45]. This list is of course very incomplete. It is intended only to give an impression of the methods that are currently used to study the Regge limit of QCD. The Regge problem is extremely difficult, and probably none of the approaches mentioned above, including the perturbative one, will by itself be sufficient to gain a full understanding. Every piece of perturbative one, will by itself be sufficient to gain a full understanding. Every piece of
information is highly welcome, and in this sense the different methods of attack should be information is highly welcome, and in this sense the different methods of attack should be
regarded as complementary. Most probably only a combined effort can eventually lead to a complete picture of the high energy limit of QCD.

After this short review of related work I would like to give an outline of my thesis. While doing so I also describe the main results of my work. I give a brief review of the well-known BFKL equation and its basic properties in the introductory chapter 1. I explain the origin of the $n$-gluon amplitudes and motivate their physical meaning in chapter 2. Here $I$ also present the integral equations that govern their behavior. This is done in great detail since the integral equations constitute the main source of information in the study of the unitarity corrections. The exploration of the five- and especially of the six-gluon amplitude requires a huge computational effort. It is therefore necessary to provide methods that allow to handle the integral equations for larger $n$ in spite of thei complexity. It is mainly in this respect that chapter 2 contains original contributions. All necessary tools to tackle the integral equations are presented in this chapter and in the two technical appendices A and B supplementing it. Chapter 3 is designed as a compact review of the three- and four-gluon amplitude. With the simple example of the four-gluon amplitude I explain the general method which is used to attack the integral equations. In brief, the method consists in splitting the amplitude under consideration into two parts, one of which has to be guessed in a smart way. This part should of course consis of known elements. The integral equations can then be used to derive a new equation 
for the remaining part. If the choice of the first part was indeed a good one the new equation allows to gain further information about the second part. The application of the vertex which will play a prominent role also in the analysis of the five- and six-gluon amplitudes. The properties of the transition vertex are presented, and the field theory structure of unitarity corrections is described in some detail. I emphasize that the very occurrence of the field theory structure is closely related to the phenomenon of reggeization in the amplitudes. Chapter 3 closes with a previously unknown property of the transition vertex. It shows that the vertex can be constructed from well-known BFKL kernels in a very intricate way. The remaining chapters consist entirely of original results. I turn to and the five-gluon amplitude can be expressed in terms of two- and forr-glen compound states. This finding constitutes a further generalization of the concept of reggeization. As a by-product the mechanism leading to reggeization in a three-gluon subsystem is found in its most general form. The knowledge of the amplitudes with up to five gluons allows to gain deeper insight into the intricate interplay of color and momentum structure in the process of reggeization. This issue is the focus of chapter 5 . In the first part of that chapter I present Ward type identities that connect a given $n$-gluon amplitudes with the $(n-1)$-gluon amplitude. They arise when one of the transverse momenta in an amplitude vanishes. In the second part of the chapter the color tensors are identifed that accompany the res. ing states. Chapter 6 deals with the six-gluon amplitude. The amplitude is split into two parts according to the method mentioned above, and a new integral equation for the remaining part is derived. I discuss its properties in detail. A piece in the equation is identified that might possibly allow an interpretation as a two-to-six gluon transition vertex. I briefly discuss the possible existence of a Pomeron-Odderon-Odderon vertex. The structure of the new equation strongly suggests that a further reggeization takes place in the four-gluon compound state Such a reggeization might give rise to a generalization of the two-to-four con pes and mention potential ways to a resolution of the problems. The problems connected with
the reggeization in the four-gluon compound state presently prohibit a full understanding the reggeization in the four-gluon compound state presently prohibit a full understanding
of the six-gluon amplitude. The new integral equation nevertheless provides evidence for some of the conjectures I have put forward in chapter 5. Finally, there are two technical appendices. Appendix A deals with contractions of tensors in su $\left(N_{c}\right)$ algebra. The method is explained and a series of identities is given that have to the best of my knowledge not been given in the literature before. Appendix B contains a combinatorial method that I have developed in order to bring certain momentum space integrals occurring in the analysis of the amplitudes to a standard form. The method is suited for implementation on a computer. I conclude this thesis with a summary and an outlook.

\section{Chapter 1}

\section{BFKL Equation and Violation of Unitarity}

In this introductory chapter we review the perturbative analysis of the Regge limit of QCD in the leading logarithmic approximation (LLA). The corresponding amplitude for elastic scattering was first derived in a massive gauge theory [10] and then also in QCD [11]. The result is known as the perturbative or BFKL Pomeron. It has since been a field of active and ongoing research. In this chapter we restrict ourselves to the basic facts about the BFKL Pomeron and we do not make any attempt on completeness. For more detailed acounts of the BFKL theory we refer to the excellent reviews [40, 46, 47]. We start with the BFKL equation and collect some basic formulae. We observe that the leading ogarithmic logarithmic approximation violates unitarity. We describe the reggeization of the gluon in the high energy limit of QCD. Finally, we explain the conformal invariance of the BFKL equation in impact parameter space.

\subsection{The BFKL equation}

The Regge limit is the kinematical region of very large center of mass energies $s$ and fixed momentum transfer $t$ of the order of a hadronic mass scale,

$$
s \gg t \simeq M_{\text {hadron }}^{2}
$$

The optical theorem relates total hadronic cross sections to the elastic forward scattering amplitude,

$$
\sigma_{\text {tot }}=\frac{1}{s} \operatorname{Im} A_{\text {el }}(s, t=0) .
$$

It is convenient to use partial wave amplitudes. In the high energy limit this amounts to performing a Mellin transformation

$$
A(s, t)=i s \int_{\delta-i \infty}^{\delta+i \infty} \frac{d \omega}{2 \pi i}\left(\frac{s}{M^{2}}\right)^{\omega} A(\omega, t)
$$

thereby changing from energy $s$ to complex angular momentum $\omega$. The high energy behavior of the total cross section is then determined by the singularities of $A(\omega, t)$ in the $\omega$-plane, the so-called Regge poles and Regge cuts. The rightmost singularity gives 
the leading contribution and is identified with the Pomeron. As it describes an elastic scattering process (see (1.2)) it carries vacuum quantum numbers.

If there is a hard momentum scale in the process the use of perturbation theory is justified. The smallness of the strong coupling constant $\alpha_{s}$ at large momentum scales can at high energy be compensated by large logarithms of the energy. This leads to the leading logarithmic approximation in which

$$
\alpha_{s} \ll 1 ; \quad \alpha_{s} \log (s) \sim 1
$$

Under this assumptions an infinite number of Feynman diagrams contributes to the scattering amplitude, and these contributions have to be resummed. This leads to the BFKL equation. The longitudinal degrees of freedom decouple in high energy scattering, and the dynamics takes place in transverse space only. The full amplitude can be written in factorized form

$$
A(\omega, t)=\int \frac{d^{2} \mathbf{k}}{(2 \pi)^{3}} \frac{d^{2} \mathbf{k}^{\prime}}{(2 \pi)^{3}} \phi_{w}\left(\mathbf{k}, \mathbf{k}^{\prime} ; \mathbf{q}\right) \phi_{1}(\mathbf{k}, \mathbf{q}) \phi_{2}\left(\mathbf{k}^{\prime}, \mathbf{q}\right) .
$$

The functions $\phi_{1}, \phi_{2}$ are the impact factors of the scattered colorless states. The color neutrality implies

$$
\phi_{1,2}(\mathrm{k}=0, \mathrm{q})=\phi_{1,2}(\mathrm{k}=\mathrm{q}, \mathrm{q})=0
$$

which is important for the infrared finiteness of the amplitude. The function $\phi_{\omega}$ can be interpreted as the partial wave amplitude for the scattering of virtual gluons with virtualities $-\mathrm{k}^{2},-(\mathrm{q}-\mathrm{k})^{2},-\mathrm{k}^{\prime 2}$, and $-\left(\mathrm{q}-\mathrm{k}^{\prime}\right)^{2}$ respectively. It is described by the BFKL equation. The BFKL equation is an integral equation in the two-dimensional space of transverse momenta and of Bethe-Salpeter type. In detail it has the form

$\omega \phi_{\omega}\left(\mathbf{k}, \mathrm{k}^{\prime} ; \mathbf{q}\right)=\phi^{0}\left(\mathbf{k}, \mathbf{k}^{\prime} ; \mathbf{q}\right)+\int \frac{d^{2} \mathbf{l}}{(2 \pi)^{3}} \frac{1}{\mathrm{l}^{2}(\mathbf{q}-\mathrm{I})^{2}} K_{\mathrm{BFKL}}(\mathrm{l}, \mathbf{q}-\mathrm{l} ; \mathrm{k}, \mathbf{q}-\mathrm{k}) \phi_{\omega}\left(\mathbf{l}, \mathbf{k}^{\prime} ; \mathbf{q}\right) \cdot(1.7)$

$\phi^{0}$ is the inhomogeneous term,

$$
\phi^{0}\left(\mathbf{k}, \mathbf{k}^{\prime} ; \mathbf{q}\right)=\frac{\delta\left(\mathrm{k}-\mathbf{k}^{\prime}\right)}{\mathbf{k}^{2}(\mathbf{q}-\mathbf{k})^{2}}
$$

The integral kernel, the so-called BFKL or Lipatov kernel, is given by

$$
K_{\mathrm{BFKL}}(\mathrm{l}, \mathrm{q}-\mathrm{l} ; \mathrm{k}, \mathrm{q}-\mathrm{k})=-N_{c} g^{2}\left[\mathrm{q}^{2}-\frac{\mathrm{k}^{2}(\mathrm{q}-\mathrm{l})^{2}}{(\mathrm{k}-\mathrm{l})^{2}}-\frac{(\mathrm{q}-\mathrm{k})^{2} \mathrm{l}^{2}}{(\mathrm{k}-\mathrm{l})^{2}}\right]
$$

$$
+(2 \pi)^{3} \mathbf{k}^{2}(\mathbf{q}-\mathbf{k})^{2}[\beta(\mathrm{k})+\beta(\mathbf{q}-\mathrm{k})] \delta^{(2)}\left(\mathrm{k}-\mathrm{k}^{\prime}\right) \cdot(1.9)
$$

The strong coupling constant is normalized to $\alpha_{s}=\frac{g^{2}}{4 \pi}$. The function $\beta$ in the kernel is defined as

$$
\beta\left(\mathrm{k}^{2}\right)=\frac{N_{c}}{2} g^{2} \int \frac{d^{2} \mathrm{l}}{(2 \pi)^{3}} \frac{\mathrm{k}^{2}}{\mathrm{l}^{2}(\mathrm{l}-\mathrm{k})^{2}} .
$$

The function

$$
\alpha\left(\mathbf{k}^{2}\right)=1+\beta\left(\mathbf{k}^{2}\right)
$$

is known as the gluon trajectory function. It passes through the physical spin 1 of the gluon at vanishing argument $\mathrm{k}^{2}=0$ because $\beta\left(\mathrm{k}^{2}=0\right)=0$. Since it is the function $\beta$ that will frequently occur throughout this thesis we call it (in obvious abuse of language) the trajectory function of the gluon as well.

The factor $\left(-N_{c}\right)$ in the BFKL kernel is a color factor. If the two gluons entering the amplitude $\phi_{w}$ are not in a color singlet state the color factor $C_{I}$ will be different. (If the two gluons are not in a color singlet state the amplitude is not infrared finite. It is then necessary to introduce a regularization. When dimensional regularization is used, for example, the calculation has to be performed in $2+\epsilon$ dimensions.) In general $C_{I}$ depends on the irreducible representation $I$ of the two gluons. If $N_{\mathrm{c}}=3$ the factor $C_{I}$ equals -3 on the irreducible representation $I$ of the two gluons. If $N_{c}=3$ the factor $C_{I}$ equals -3 ,
$-\frac{3}{2},-\frac{3}{2}, 0,1$ for the irreducible representations $1,8_{\mathbf{A}}, 8_{\mathbf{S}}, 10+\overline{10}, 27$, respectively. In this thesis we will use the symbol $K_{\mathrm{BFKL}}$ for the BFKL kernel only if the two gluons are in a color singlet.

The complex angular momentum $\omega$ acts as an energy variable in the BFKL equation. It can be shown to be conjugate to rapidity which thus acquires the meaning of a time variable in the BFKL equation.

The general form of the solution of the BFKL equation can be derived from the integral equation by iteration. Accordingly, the elastic scattering amplitude at high energies has in LLA the structure of a gluon ladder in the $t$-channel,

$$
s \rightarrow \infty \searrow=\sum_{\substack{\text { number } \\ \text { of rungs }}} \boxminus
$$

and the ladder rungs represent BFKL kernels.

For vanishing momentum transfer $t$ one obtains the BFKL equation in the forward direction. It can be diagonalized by power functions

$$
e^{(\nu, n)}(\mathrm{k})=2 \pi \sqrt{2}(\mathbf{k})^{-\frac{3}{2}-i \nu} e^{-i n \phi}
$$

with $\nu \in \mathbb{R}, n \in \mathbb{Z}$. The eigenvalues are

$$
\chi(\nu, n)=\frac{N_{c} \alpha_{s}}{\pi}\left[2 \psi(1)-\psi\left(\frac{1+|n|}{2}+i \nu\right)-\psi\left(\frac{1+|n|}{2}-i \nu\right)\right],
$$

where $\psi$ is the logarithmic derivative of the Euler $\Gamma$-function. The full solution of the BFKL equation consequently reads

$$
\phi_{\omega}\left(\mathrm{k}, \mathrm{k}^{\prime}\right)=\sum_{n=-\infty}^{+\infty} \int_{-\infty}^{+\infty} \frac{d \nu}{2 \pi} \frac{1}{\omega-\chi(\nu, n)} e^{(\nu, n)}(\mathrm{k}) e^{(\nu, n) *\left(\mathrm{k}^{\prime}\right) .}
$$

The eigenfunctions for non-zero momentum transfer $t$ are only known in impact parameter space.

To conclude this section we mention the $t$-channel reggeon unitarity relation for the BFKL amplitude. Let $C\left(\omega ; \mathbf{k}, \mathbf{k}^{\prime} ; q\right)$ be the amputated BFKL amplitude, i. e. the amplitude $\phi$ without the reggeon propagator $\left(\omega-\beta\left(\mathrm{k}^{2}\right)-\beta\left((\mathrm{q}-\mathrm{k})^{2}\right)\right)^{-1}$

$$
C\left(\omega ; \mathrm{k}, \mathrm{k}^{\prime} ; \mathbf{q}\right)=\left(\omega-\beta\left(\mathrm{k}^{2}\right)-\beta\left((\mathbf{q}-\mathbf{k})^{2}\right)\right) \phi_{\omega}\left(\mathrm{k}, \mathrm{k}^{\prime} ; \mathbf{q}\right)
$$


with $t=-\mathrm{q}^{2}$. After a continuation to the physical region of the $t$-channel it is the possible to show [10] with the help of the BFKL equation that

$$
\operatorname{disc}_{\omega} C=\int d^{2} \mathbf{l}(2 \pi)^{3} \frac{1}{1^{2}} \frac{1}{(\mathbf{q}-1)^{2}} \delta\left(\omega-\beta\left(\mathbf{l}^{2}\right)-\beta\left((\mathbf{q}-\mathbf{l})^{2}\right)\right)
$$$$
\times C(\omega ; \mathrm{k}, \mathrm{l} ; \mathrm{q}) C^{*}\left(\omega ; \mathrm{l}, \mathrm{k}^{\prime} ; \mathrm{q}\right) .
$$

The right hand side can be understood as a unitarity integral for the two-gluon amplitud $C$. We will come back to the significance of such $t$-channel reggeon unitarity relations in section 2.1 of the next chapter.

\subsection{Violation of unitarity}

The high energy asymptotics of the BFKL amplitude is determined by the rightmost singularity in the plane of complex angular momentum $\omega$. Since the function $\chi(\nu, n)$ (see (1.14)) decreases with increasing $n$ one can neglect the contributions with $n \neq 0$. Furthe it is possible to make an expansion of $\chi(\nu, 0)$ in $\nu$ around zero to find the leading singularity in (1.15). It leads to a power-like growth of the amplitude

$$
A \sim s^{\left(1+\omega_{\mathrm{BFKL}}\right)},
$$

and the exponent as obtained from the calculation outlined above is

$$
\omega_{\mathrm{BFKL}}=\frac{\alpha_{s} N_{c}}{\pi} 4 \ln 2 \simeq 0.5
$$

Consequently, the total cross section in the leading logarithmic approximation grows like

$$
\sigma_{\text {tot }} \sim s^{\omega_{\text {BFKL }}} .
$$

This result is in conflict with the Froissart-Martin theorem [14, 15]. This theorem de rives a bound on the total hadronic cross section from unitarity. The theorem is thus a consequence of a deep principle of field theory. In detail, the Froissart-Martin bound is

$$
\sigma_{\text {tot }} \leq \text { const. } \log ^{2}(s) \text {. }
$$

A power-like growth will eventually violate this bound at asymptotically large energies. This observation is the starting point of the considerations in this thesis. Before we come to this central issue in the next chapter we will describe a few more properties of the BFKL equation.

\subsection{Reggeization of the gluon}

The phenomenon of reggeization in non-abelian gauge theories was discovered by Lipatov [12] in 1976 when he made the following striking observation. The $t$-channel exchange in the BFKL equation carrying the quantum numbers of a gluon, i. e. a color octet ${ }^{1}$ exchange, gives rise to a special solution. For antisymmetric color octet exchange the color factor

\footnotetext{
${ }^{1}$ We speak of 'octet' to mean the adjoint representation also for general $N_{c}$.
}

in the kernel $K_{\mathrm{BFKL}}$ is $N_{c} / 2$ instead of $N_{c}$. (In this color representation the amplitude is not infrared finite and a regularization has to be applied.) Let us further assume that the in homogeneous term $\phi_{0}$ is a function of $\left(k_{1}+k_{2}\right)$. Then the equation exhibits the solution

$$
\phi^{8_{A}}\left(k_{1}+k_{2}\right)=\frac{\phi_{0}^{8_{A}}\left(k_{1}+k_{2}\right)}{\omega-\beta\left(k_{1}+k_{2}\right)} .
$$

This solution has a pole and thus can be interpreted as describing the propagation of a single particle with momentum $\left(\mathrm{k}_{1}+\mathrm{k}_{2}\right)$ and the quantum numbers of a gluon. In a sense the gluon turns out to be a bound state of two gluons here. The fact that the gluon is a composite state of gluons is often termed 'bootstrap'. It indicates that the correct degrees of freedom in high energy QCD are not elementary gluons but so-called reggeized gluons. The reggeized gluon can be understood as a collective excitation of the Yang-Mills the reggeized gluon field contribute to the reggeized gluon as a collective excitation (see

When we interchange the two gluons in the color octet amplitude above we find that its sign changes. This fact gives rise to the notion of signature. It characterizes the behavior under the exchange of two gluons, that is the simultaneous interchange of color and momentum labels. The reggeized gluons obviously carries negative signature.

\subsection{Conformal invariance in impact parameter space}

One of the most interesting properties of the BFKL equation is the conformal invariance in impact parameter space. It was proven in [13] and made it possible to find the solutions of the BFKL equation in the non-forward direction as well.

The Fourier transformation of the partial wave amplitude $\phi_{\omega}$ is defined as

$$
\delta\left(\mathrm{q}-\mathrm{q}^{\prime}\right) \phi_{\omega}\left(\mathrm{k}, \mathrm{k}^{\prime} ; \mathrm{q}\right)=\int d^{2} \rho_{1} d^{2} \rho_{2} d^{2} \rho_{1^{\prime}} d^{2} \rho_{2^{\prime}} \phi_{\omega}\left(\rho_{1}, \rho_{2} ; \rho_{1^{\prime}}, \rho_{2^{\prime}}\right) \times
$$

$$
\times \exp \left(i \mathbf{k} \rho_{1}+i(\mathbf{q}-\mathbf{k}) \rho_{2}-i \mathbf{k}^{\prime} \rho_{\mathbf{1}^{\prime}}-i\left(\mathbf{q}^{\prime}-\mathbf{k}^{\prime}\right) \rho_{2^{\prime}}\right)(1.23)
$$

Fourier transformation space defines the BFKL equation in two-dimensional impact parameter space. It is convenient to write the vectors in this space in complex notation, $\rho=\rho_{x}+i \rho_{y}$. The BFKL kernel can then be shown to be invariant under the transformations

$$
\rho \rightarrow \rho^{\prime}=\frac{a \rho+b}{c \rho+d} ; \quad a d-b c=1 .
$$

These transformations are characterized by the group

$$
\left(\begin{array}{ll}
a & b \\
c & d
\end{array}\right) \in \mathrm{SL}(2, \mathbb{C}) / Z_{2}
$$

i. e. the group of projective conformal transformations. An arbitrary conformal transformation can always be obtained as the superposition of the following basic transformations:

$$
\begin{array}{ll}
\text { translations: } & \rho \rightarrow \rho+b \\
\text { rotations: } & \rho \rightarrow a \rho ; \quad|a|=1 \\
\text { dilatations: } & \rho \rightarrow \lambda \rho ; \quad \lambda \in \mathbb{R}_{+} \\
\text {inversions: } & \rho \rightarrow \rho^{-1} .
\end{array}
$$


This observation allows to expand the solution of the BFKL equation in conformal partial waves,

$$
\begin{aligned}
\phi_{\omega}\left(\rho_{1}, \rho_{2} ; \rho_{1^{\prime}}, \rho_{2^{\prime}}\right)= & \sum_{n=-\infty}^{+\infty} \int \frac{d \nu}{2 \pi} \frac{16 \nu^{2}+4 n^{2}}{\left[4 \nu^{2}+(n-1)^{2}\right]\left[4 \nu^{2}+(n+1)^{2}\right]} \\
& \times \frac{1}{\omega-\chi(\nu, n)} \int d^{2} \rho_{0} E^{(\nu, n)}\left(\rho_{10}, \rho_{20}\right) E^{(\nu, n) *}\left(\rho_{1^{\prime} 0}, \rho_{2^{\prime} 0}\right) \cdot(1.26)
\end{aligned}
$$

\section{Chapter 2}

Here we use $\rho_{i j}=\rho_{i}-\rho_{j}$. The representation functions $E^{(\nu, n)}$ are given by

$$
E^{(\nu, n)}\left(\rho_{10}, \rho_{20}\right)=c(\nu, n)\left(\frac{\rho_{12}}{\rho_{10} \rho_{20}}\right)^{h}\left(\frac{\rho_{12}^{*}}{\rho_{10}^{*} \rho_{20}^{*}}\right)^{\bar{h}}
$$

with a constant $c(\nu, n)$. The coordinate $\rho_{0}$ represents an additional parameter of the functions $E^{(\nu, n)}$. The parameters $h$ and $\bar{h}$ are the conformal weights of the representation, and we have

The combination

$$
\bar{h}=1-h^{*} .
$$

is the integer conformal spin of the state and its scaling dimension is given by

$$
h+\bar{h}=1+2 \nu \in \mathbb{R} .
$$

These two relations also explain the notation $E^{(\nu, n)}$ for the representation functions. The representation functions can be understood as three-point correlation functions of a conformal field theory [40],

$$
E^{(\nu, n)}\left(\rho_{10}, \rho_{20}\right)=\left\langle\phi_{0,0}\left(\rho_{1}, \bar{\rho}_{1}\right) \phi_{0,0}\left(\rho_{2}, \bar{\rho}_{2}\right) O_{h, h}\left(\rho_{0}, \bar{\rho}_{0}\right)\right\rangle,
$$

with the identities $(1.29),(1.30)$ relating $\nu, n$, and $h$. The operators $\phi_{0,0}$ can be interpreteted as elementary fields representing reggeized gluons. They have zero conformal weight. The operator $O_{h, h}$ represents a composite state of two reggeized gluons that emerges from the dynamics of the theory.

We limit ourselves to this short review of the basic properties of the BFKL equation in impact parameter space. Conformal invariance has far-reaching consequences. It places strong constraints on the correlation functions of the theory, for example. We will come back to the questions related to conformal invariance in the BFKL equations and in the unitarity corrections in chapter 3 .

\section{Integral Equations for $n$-gluon Amplitudes}

The aim of this chapter is to set the stage for the analysis of unitarity corrections we will carry out in this thesis. The physical process motivating our investigations is the scattering of highly virtual photons at very large center-of-mass energy. Here the main objects to be studied are partial wave amplitudes describing the production of a given number $n$ of reggeized gluons in the $t$-channel. The approach we will pursue in this thesi heavily relies on the use of integral equations for the $n$-gluon amplitudes. We provide all necessary tools for their study in this chapter. In the first section we concentrate on the $n$-gluon amplitudes and motivate their physical meaning. After that we turn to the mplitudes we treat them in the subsequent sections. In the literature the integral equations have so far been treated only for up to four gluons. We want to study amplitudes with up to six reggeized gluon and therefore have to extend the equations accordingly. The complexity of the problen rapidly increases with the number of gluons in the amplitude under consideration. It is thus highly desirable to develop suitable methods and to cast the formalism into a form that allows to treat the problem in spite of its complexity. We pay special attention to this aspect throughout this chapter and supplement it in this respect with the two appendices $\mathrm{A}$ and $\mathrm{B}$.

\subsection{The $n$-gluon amplitudes}

As the physical process to consider we choose the scattering of highly virtual photons at very large center-of-mass energy and fixed momentum transfer. This process is not only of theoretical interest. The total cross section of $\gamma^{*} \gamma^{*}$-scattering can be measured at the the planned next linear collider (NLC) facility where it occurs as a subprocess in electronpositron $\left(e^{+} e^{-}\right)$scattering. There the total $\gamma^{*} \gamma^{*}$ cross section will be sizeable and allow or a very clean test of the the high energy regime of $\mathrm{QCD}[5,6]$. From a theoretical point of view the process is extremely interesting since the highly virtual photon with invariant C $Q$ provides harc QCD we are thus in a position to use perturbation theory as the framework for ou 
colliding particles have a large virtuality. Moreover, in $\gamma^{*} \gamma^{*}$-scattering we are not plagued with the theoretical difficulties arising from the genuinely non-perturbative internal structure of the proton present in deep-inelastic electron-proton scattering. There is, however, the problem of diffusion of momenta in the process. We will come back to this problem momentarily. Already here we can state that the virtualities of the two photons should be chosen to be large and of comparable size.

We are interested in the hadronic total cross section of $\gamma^{*} \gamma^{*}$-scattering that is related to the elastic forward scattering amplitude via the optical theorem. In high energy scattering the integrations over longitudinal momenta become trivial and the dynamics of the process essentially takes place in the two-dimensional space of transverse momenta. At high energy the small value of the strong coupling constant $\alpha_{s}$ can be compensated by large logarithms of the energy $s$, and the corresponding contributions have to be resummed. In leading logarithmic approximation the elastic forward amplitude of $\gamma^{*} \gamma^{*}$-scattering is therefore described by the BFKL amplitude, that is by the exchange of a compound state of two reggeized gluons in the $t$-channel. The latter are coupled to the photons via (light) quarks, i. e. each of the photons actually splits into a quark-antiquark par which then undergos in interaction media the powe bound (1.21) and results in a non-unitary scattering amplitude. (Strictly speaking, the Froissart-Martin theorem was proven only for the scattering of on-shell hadrons. It is widely believed to hold for the scattering of virtual photons as well, but a concise proo is still lacking in this case.) The restoration of unitarity necessitates the inclusion of a certain class of higher-order corrections to the leading logarithmic approximation. This is the main point motivating the introduction of $n$-gluon amplitudes. Eventually, we want to find out how unitarity will be restored in the Regge limit of QCD. We will therefore be concerat with asymptotically large center-of mass attention to phenomenological applications in this the Though, it is well conceivable that the first unitarity corrections might already be important for the interpretation of the data to be taken at the NLC.

The use of the leading logarithmic approximation bears a problem concerning the use of perturbation theory that is not obvious on first sight. When leading logarithms in the energy $s$ are resummed the longitudinal momenta along the gluon ladder are strongly ordered. But there is no constraint on the transverse momenta besides energy-momentum conservation. Due to this there is a diffusion of transverse momenta $[48,49]$ that can ing logarithms in the momentum scale $Q^{2}$ which results in the well-known DokshitzerGribov-Lipatov-Altarelli-Parisi (DGLAP) equation [50]. In the latter case the transverse momenta are strongly ordered along the ladder and diffusion does not take place. Diffusion does occur in $\gamma^{*} \gamma^{*}$-scattering at high energy that is of interest to us. The contribution of small momenta is still not small at the NLC, for instance. But the situation is quite favorable at least from a theoretical point of view. Choosing the virtualities of the colliding photons large enough we can make the contribution from the dangerous low-momentum region very small. This makes a perturbative calculation quite trustworthy. Therefore $\gamma^{*} \gamma^{*}$ scattering is arguably the best posible process for the theotical investigan thestigation of high energy QCD. Ideally, the virtualities of the two photons should be of comparable size
to suppress DGLAP evolution. The method suited to restore unitarity in the perturbative approach is known as the generalized leading logarithmic approximation, and we will pursue this method here. It constitutes an approximation scheme in which a minimal set of non-leading corrections is identified that leads to a unitary amplitude. In essence, the minimal set of contributions required here comprises subleading logarithmic corrections with a larger number $n$ of reggeized gluons in the $t$-channel. These are what we call unitarity corrections. As we will see in a moment, one has to sum over all possible $n$ to fulfill the requirement of unitarity. Quark exchanges in the $t$-channel are always suppressed by powers of the energy $s$ with respect to the corresponding gluon exchanges. They are not taken into account in the generalized leading logarithmic approximation. Let us now try to understand how such corrections may result in a unitary amplitude. The corrections will in general give contributions to the scattering amplitude that grow like powers of the center-of-mass energy $s$. The respective exponent will depend on the number $n$ of reggeized gluons that are exchanged and, like in the BFKL amplitude, be related to the spectrum of the $n$-gluon state. Obviously, a finite sum of powers cannot grow slower with energy than a logarithm. This already makes clear that we have to take in to account an infinite set of contributions. We have to sum over all posible corrections with $n$ gluons. Let us asume that only We have to sur corrections with an even number $n$ of gluons give rise to a new exponent. (We will find
good reasons for this assumption in the course of our investigations.) The sign of these good reasons for this assumption in the course of our investigations.) The sign
corrections is expected to alternate. The full amplitude then acquires the form

$$
A(s)=i \sum_{n \in 2 \mathbb{N}}^{\infty}(-1)^{\frac{n}{2}} A_{n} s^{\omega_{n}} \leq \text { const. } \log ^{2}(s)
$$
with some coefficients $A_{n}$, and now it is well possible that in the high energy limit the
amplitude is bounded by a logarithm as indicated. The knowledge of how exactly the mechanism sketched in (2.1) works is admittedly rather limited. In fact only the first. mechanism sketched in (2.1) works is admittedly rather limited. In fact only the first exponent, i. e. the BFKL exponent $\omega_{B F K L}$ (1.19), is known. We refer the reader to [24]
for a more detailed description of how the second term in the series (2.1) can be obtained once the spectrum of the four-gluon state is known.

The most complete approach to a systematic treatment of unitarity corrections was formulated by Bartels $[17,18,19]$. Its aim is to arrive at an effective description of QCD in the Regge limit in the spirit of a reggeon field theory [2,3], the requirement of unitarity being built in from the very beginning. Of course, the BFKL amplitude with its two $t$-channel gluons should be incorporated into the whole approach as the lowest its two $t$-channel gluons should be incorporated into the whole approach as the lowest BFKL amplitude but now with $n$ reggeized gluons in the $t$-channel. In section 1.1 we have seen the $t$-channel reggeon unitarity equation for the BFKL amplitude, cf. (1.17). It is valid when the amplitudes are continued to positive $t$, that is to the physical region of the $t$-channel. Its essence can be summarized symbolically as

$$
\operatorname{disc}_{\omega} A(\omega, t) \sim C_{2} C_{2}^{*},
$$

where $C_{2}$ is an amputated amplitude. We include a reggeon propagator to arrive at $D_{2}=C_{2}\left(\omega-\beta\left(\mathbf{k}_{1}\right)-\beta\left(\mathbf{k}_{2}\right)\right)^{-1}$. We have chosen a new symbol $D_{2}$ for the BFKL amplitude here since we are now considering the special physical process of $\gamma^{*} \gamma^{*}$-scattering. The amplitude $D_{2}$ obeys the BFKL equation (1.7) with a special choice of the inhomogeneous term, namely the coupling of the two gluons to the photons via a quark loop. The unitarity 
relation (2.2) can be generalized to include $n$-gluon intermediate states. Symbolically, the generalization has the form

$$
\operatorname{disc}_{\omega} A(\omega, t) \sim \sum_{n=2}^{\infty} C_{n} C_{n}^{*}
$$

We include a reggeon propagator to find $D_{n}=C_{n}\left(\omega-\sum_{i=1}^{n} \beta\left(\mathrm{k}_{i}\right)\right)^{-1}$. Here the $D_{n}$ describe the production of $n$ on-shell gluons in the $t$-channel. They are non-amputated amplitudes, i.e. have propagators on the external gluon lines. The correct treatment of $t$-channel unitarity relations including multi-particle amplitudes is highly non-trivial. To our knowledge the most complete survey of this extensive technology is [51], and the reader is referred to that reference for the details. We will content ourselves here with having motivated the physical meaning of the $n$-gluon amplitudes $D_{n}$ we are going to study. Below we will outline the formal definition of the amplitudes $D_{n}$.

Once we take into account subleading corrections with more reggeized gluons in the $t$-channel and consider multi-particle amplitudes like the $n$-gluon amplitudes $D_{n}$ there exist of course subchannels of the scattering amplitude and we have to care about unitary also in the subchannels. The approach initiated by Bartels is designed to ensure unitarity not only in the lirect chan gluons in the $t$-channel gluons is not conserved. Due to that the set of integral equation for the $n$-gluon amplitudes is turned into a tower of coupled equations including numberchanging integral kernels. (A detailed description of the integral equations will follow in section 2.2.)

The non-conservation of the number of reggeized gluons in the $t$-channel evolution contrasts sharply with the situation in the Bartels-Kwieciński-Praszalowicz (BKP) equations $[18,20]$. The latter describe the $t$-channel evolution of a compound state of a fixed num 118 , 20]. The later desibe the equivalent to a completely integrable model [29], namely the XXX-1 conformal SL $(2, \mathbb{C})$ spin $s=0$ [30]. Although the BKP equations do not apply directly to our $n$-gluon amplitudes $D_{n}$ they will play an important role in the effective field theory of unitarity corrections that we are heading for. As we will explain in more detail in section 3.3 there will be different elements in the effective field theory. First we will have $n$-particle Green functions, that is number-conserving elements. Their behavior will be governed by the BKP equations. In addition, there will be number-changing elements which we will call vertices. They arise as a unique feature of the approach pursued here, and turn the quantum mechanical problem described by the BKP equations into a quantum field theory.

We will now outline the formal definition of the $n$-gluon amplitudes $D_{n}$. The way We amplitudes $D_{n}$ are defined is inspired by Regge theory. A condensed but still rather extensive description of the methods that have to be used here can be found in [52], more complete reviews are contained in [53] and [54]. The procedure starts from a physical $2+n$ multi-particle scattering process. One identifies certain kinematical variables with the use of so-called Toller diagrams and hexagraphs. After defining partial waves one can

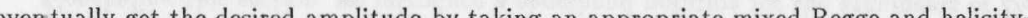
it is in principle possible we will not cany out this program for the amplitudes under consideration in this thesis. The procedure becomes technically complicated very quickly with the increasing number of gluons in the amplitude. Moreover the physical processes we would have to start with for larger $n$ are very artifical and of purely academic interest. However, the method outlined here appears to be very natural for a special phenomenological application of the four-gluon amplitude. In [22] a part of the four-gluon amplitude $D_{4}$ in which the four gluons form two pairs of color singlets wa used for the description of the process of high-mass diffractive dissociation in deep inelastic electron-proton scattering. The rapidity gap between the proton and the diffractively produced system is caused by a colorless exchange between the proton and the photon. The latter is modelled by a two-gluon exchange. In the amplitude for this process the initial state is therefore indeed a three-particle state, and the cross-section takes the form of a three-to-three scattering process. In [22] the appropriate limit for this process was identified as the well-known triple Regge limit in which $s \gg M^{2} \gg Q^{2} \gg \Lambda_{Q C D}^{2}$, where $M^{2}$ is the invariant mass of the diffractively produced particles.

The $n$-gluon amplitudes are defined to have as external lines two photons and $n$ gluons. The photons are coupled to the gluons via their splitting into a quark-antiquark pair to which the gluons are attached. Being $n$-gluon amplitudes the $D_{n}$ carry as arguments color labels $a_{i}$ in addition to the transverse momenta $\mathbf{k}_{i}$ of the gluons. The color label correspond to generators $t^{a_{i}}$ of the gauge group $\mathrm{SU}\left(N_{c}\right)$ in the adjoint representation. As partial waves the $D_{n}$ have also as an argument the complex angular momentum $\omega$. Since all $D_{n}$ will carry the same argument $\omega$ we will suppress it in our notation. In our notation we will suppress the photon momenta as well. The $n$-gluon amplitudes are thu characterized as

$$
D_{n}^{a_{1} \ldots a_{n}}\left(\mathbf{k}_{1}, \ldots, \mathbf{k}_{n}\right) \text {. }
$$

The transverse gluon momenta $\mathbf{k}_{i}$ in the amplitude are all chosen to be outgoing. The $D_{n}$ are non-amputated amplitudes, i. e. they have propagators for the outgoing reggeized gluons. Further they are multiply-cut amplitudes. We take discontinuities in the $n-1$ energy variables defined from one photon and the $i$ first gluons $(1 \leq i \leq n-1)$,

$$
s_{i}=\left(p_{\gamma^{*} ; 1}+\sum_{j=1}^{i} p_{j}\right)^{2} .
$$

Here $p_{\gamma^{*} ; 1}$ and $p_{j}$ are the four-momenta of the left photon and the gluons, respectively. The amplitudes $D_{n}$ can be defined for the non-forward direction

$$
\sum_{i=1}^{n} k_{i} \neq 0
$$

as well. All results in this thesis will hold for the non-forward direction, and we will not mention this in each case separately.

The simplest of the $n$-gluon amplitudes is $D_{2}$. It is identical with the well-known BFKL amplitude discussed in chapter 1 (there it was denoted $\phi$ ). There the inhomogeneous term in the BFKL equation was not specified. In $D_{2}$ it is given by the lowest order coupling of the two $t$-channel gluons to the virtual photons through a quark loop. The two outgoing gluons in the BFKL amplitude $D_{2}^{a_{1} a_{2}}$ are in a color singlet such that we can factorize the color structure and define the momentum part $D_{2}$ by

$$
D_{2}^{a_{1} a_{2}}\left(\mathbf{k}_{1}, \mathbf{k}_{2}\right)=\delta_{a_{1} a_{2}} D_{2}\left(\mathbf{k}_{1}, \mathbf{k}_{2}\right) .
$$

We repeat here two simple but very important properties of the momentum part (see (2.7)) of the BFKL amplitude. The first is that it vanishes when one of its momentum argument vanishes,

$$
\left.D_{2}\left(\mathbf{k}_{1}, \mathbf{k}_{2}\right)\right|_{\mathbf{k}_{1}=0}=\left.D_{2}\left(\mathbf{k}_{1}, \mathbf{k}_{2}\right)\right|_{\mathbf{k}_{2}=0}=0
$$


The second is the symmetry in its two momentum arguments,

$$
D_{2}\left(\mathbf{k}_{1}, \mathbf{k}_{2}\right)=D_{2}\left(\mathbf{k}_{2}, \mathbf{k}_{1}\right) \text {. }
$$

Concluding this section, we introduce a shorthand notation for the arguments of $D_{2}$. Later we will use it for the arguments of other functions as well. In the case that an argument of a function, say $D_{2}$, is a sum of two or more transverse momenta we will only give the indices of these momenta, and a string of indices stands for the sum of the corresponding momenta ${ }^{1}$. So we have for example

$$
D_{2}(12,3)=D_{2}\left(\mathbf{k}_{1}+\mathbf{k}_{2}, \mathbf{k}_{3}\right) \text {. }
$$

\subsection{Integral equations}

In the preceding section we have introduced the main objects of our investigations, namely the $n$-gluon amplitudes. In the present section we get to know the source from which we will draw information about the amplitudes. This source of information is provided by a set of integral equations. Hidden in them is an amazingly rich structure, and they will help us to gain insight into the principles underlying the Regge limit of QCD.

The $n$-gluon amplitudes $D_{n}$ obey a tower of coupled integral equations. They were derived in [19] by means of $s$-channel dispersion relations. Not surprisingly, they bear some resemblance to the BFKL equation. They are equations in two-dimensional transverse momentum space. Like the BFKL equation they describe the $t$-channel evolution of the amplitudes under investigation. In this evolution, the complex angular momentum $\omega$ again plays the role of an energy variable. Its conjugate is rapidity, and it acquires the meaning of the time-like variable in the evolution.

The integral equation for the two-gluon amplitude $D_{2}^{a_{1} a_{2}}$ is of course identical to the BFKL equation,

$$
\left(\omega-\sum_{i=1}^{2} \beta\left(\mathbf{k}_{i}\right)\right) D_{2}^{a_{1} a_{2}}=D_{(2 ; 0)}^{a_{1} a_{2}}+K_{2 \rightarrow 2}^{\{b\} \rightarrow\{a\}} \otimes D_{2}^{b_{1} b_{2}} .
$$

We have moved the trajectory functions to the left hand side of the equation to make the generalization to larger $n$ more transparent. The inhomogeneous term $D_{(2 ; 0)}$ denotes the lowest order coupling of the two gluons to the photons via the quark loop. The quark loop will be the subject of section 2.4. The integral kernel $K_{2 \rightarrow 2}^{\{b\} \rightarrow\{a\}}$ is, roughly speaking, the BFKL kernel (1.9) without the gluon trajectory functions $\beta$. An exact definition of the integral kernels will be given in section 2.6. The superscript $\{b\} \rightarrow\{a\}$ corresponds to the color labels of the in- and outgoing gluons. The convolution symbol $\otimes$ stands for an integral $\int \frac{d^{2} 1}{(2 \pi)^{3}}$ over the loop momentum and a propagators $\frac{1}{1^{2}}$ for each of the two gluons entering the kernel from above.

The integral equation for the three-gluon amplitude $D_{3}^{a_{1} a_{2} a_{3}}$ has the form

$\left(\omega-\sum_{i=1}^{3} \beta\left(\mathbf{k}_{i}\right)\right) D_{3}^{a_{1} a_{2} a_{3}}=D_{(3 ; 0)}^{a_{1} a_{2} a_{3}}+K_{2 \rightarrow 3}^{\{b\} \rightarrow\{a\}} \otimes D_{2}^{b_{1} b_{2}}+\sum K_{2 \rightarrow 2}^{\{b\} \rightarrow\{a\}} \otimes D_{3}^{b_{1} b_{2} b_{3}} \cdot$ (2.12)

${ }^{1}$ The notation is safe as long as we do not go beyond $n=9$ gluons. This, however, is very unlikely for the foreseeable future.
The inhomogeneous term $D_{(3 ; 0)}$ is now the quark loop with three gluons attached to it. In (2.12) we find for the first time a new kernel in the equation. $K_{2 \rightarrow 3}^{\{b\} \rightarrow\{a\}}$ is a transition kernel from two to three reggeized gluons. The second term on the right hand side of the equation therefore tells us that at some point in the $t$-channel evolution we can have a transition from two to three gluons. The last term describes the evolution of a systen of three gluons, and the sum extends over all pairwise interactions of the three reggeized gluons via the kernel $K_{2}^{\{b\} \rightarrow\{a\}}$. Let us look at the term in which the first and second gl uon interact via a kernel. In this term the momentum and the color label of the third gluon interact via a kernel. In this term the momentum and the color label of the third gluon
are not affected. The kernel should thus be understood to contain a factor $\delta_{a_{3} b_{3}}$. The are not affected. The kernel should thus be understood to contain a factor $\delta_{a_{3} b_{3}}$. The
symbol $\otimes$ denotes again the integration over the loop momentum in the first two gluons and propagator factors for each of them. The other terms are obtained in analogy.

The equations for higher $n$ are built in a very similar way. They contain as the respective inhomogeneous term the lowest order coupling of $n$ gluons to the quark loop. We denote this lowest order term as $D_{(n, 0)}$. A detailed discussion of the quark loop and contain also higher transition kernels $K_{2 \rightarrow m}^{\{b\} \rightarrow\{a\}}$ from two to $m$ gluons. A general formula for arbitrary $m$ as well as the explicit formulae for $m \leq 6$ are contained in section 2.6 .

Since we will make use of the integral equations for up to $n=6$ in this thesis, we now state them explicitly. The general rule should then be obvious. For $n=4$ we have

$$
\left(\omega-\sum_{i=1}^{4} \beta\left(\mathbf{k}_{i}\right)\right) D_{4}^{a_{1} a_{2} a_{3} a_{4}}=D_{(4 ; 0)}^{a_{1} a_{2} a_{3} a_{4}}+K_{2 \rightarrow 4}^{\{b\} \rightarrow\{a\}} \otimes D_{2}^{b_{1} b_{2}}+\sum K_{2 \rightarrow 3}^{\{b\} \rightarrow\{a\}} \otimes D_{3}^{b_{1} b_{2} b_{3}}
$$$$
+\sum K_{2 \rightarrow 2}^{\{b\} \rightarrow\{a\}} \otimes D_{4}^{b_{1} b_{2} b_{3} b_{4}},
$$

for $n=5$ the equation is

$$
\begin{aligned}
\left(\omega-\sum_{i=1}^{5} \beta\left(k_{i}\right)\right) D_{5}^{a_{1} a_{2} a_{3} a_{4} a_{5}}= & D_{(5 ; 0)}^{a_{1} a_{2} a_{3} a_{4} a_{5}}+K_{2 \rightarrow 5}^{\{b\}-\{a\}} \otimes D_{2}^{b_{1} b_{2}} \\
& +\sum K_{2 \rightarrow 4}^{\{b\} \rightarrow\{a\}} \otimes D_{3}^{b_{1} b_{2} b_{3}}+\sum K_{2 \rightarrow 3}^{\{b\}-\{a\}} \otimes D_{4}^{b_{1} b_{2} b_{3} b_{4}} \\
& +\sum K_{2 \rightarrow 2}^{\{b\} \rightarrow\{a\}} \otimes D_{5}^{b_{1} b_{2} b_{3} b_{4} b_{5}}
\end{aligned}
$$

and finally for $n=6$ the integral equation reads

$$
\begin{aligned}
\left(\omega-\sum_{i=1}^{6} \beta\left(\mathrm{k}_{i}\right)\right) D_{6}^{a_{1} a_{2} a_{3} a_{4} a_{5} a_{6}}= & D_{(6 ; 0)}^{a_{1} a_{2} a_{3} a_{4} a_{5} a_{6}}+K_{2 \rightarrow 6}^{\{b\} \rightarrow\{a\}} \otimes D_{2}^{b_{1} b_{2}} \\
& +\sum K_{2 \rightarrow 5}^{\{b\} \rightarrow\{a\}} \otimes D_{3}^{b_{1} b_{2} b_{3}}+\sum K_{2 \rightarrow 4}^{\{b\} \rightarrow\{a\}} \otimes D_{4}^{b_{1} b_{2} b_{3} b_{4}} \\
& +\sum K_{2 \rightarrow 3}^{\{b\} \rightarrow\{a\}} \otimes D_{5}^{b_{1} b_{2} b_{3} b_{4} b_{5}} \\
& +\sum K_{2 \rightarrow 2}^{\{b\} \rightarrow\{a\}} \otimes D_{6}^{b_{1} b_{2} b_{3} b_{4} b_{5} b_{6}} .
\end{aligned}
$$

Here we again have to explain the meaning of the convolutions and the summation symbols. In short, the sums contain all combinations of the respective amplitudes and kernels in which the $t$-channel gluons do not cross. Before we give an example of the combinatorics we write the integral equations in pictorial language. That makes the integral equations 
very catchy which is highly desirable since they are at the very center of the discussion in the following chapters.

$$
\begin{aligned}
& \left(\omega-\sum_{i=1}^{2} \beta\left(\mathbf{k}_{i}\right)\right) D_{1} D_{1}=D_{(2 ; 0)}+D_{2} \\
& \left.\left(\omega-\sum_{i=1}^{3} \beta\left(\mathbf{k}_{i}\right)\right) \prod_{\prod}^{D_{3}}=\prod_{\Pi 1}^{D_{(3 ; 0)}}\right)^{-D_{2}}+\sum \frac{D_{3}}{H T}
\end{aligned}
$$

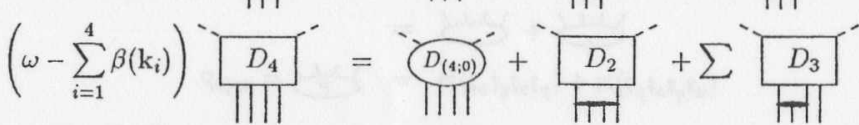

$$
\begin{aligned}
& +\sum D_{H}
\end{aligned}
$$

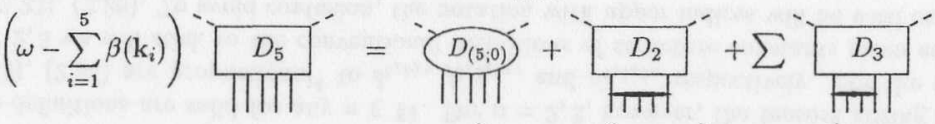

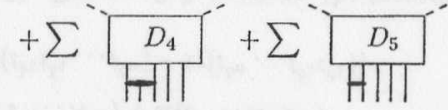

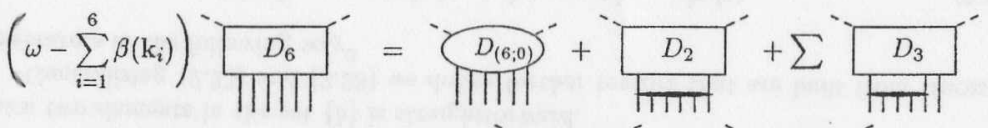

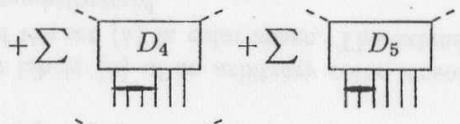

$$
\begin{aligned}
& +\sum \stackrel{D_{6}}{H \pi \|}
\end{aligned}
$$

In each diagram only two gluon lines from the amplitudes enter a kernel. An integration $\int \frac{d^{2} 1}{(2 \pi)^{3}}$ over the loop momentum and a propagator $\frac{1}{1^{2}}$ for each of the two gluons entering the kernel from above are implied again. The momenta and color labels of the other gluons are not changed. With the help of the pictorial notation it is also very easy to understand which combinations of amplitudes and kernels have to be convoluted such that $t$-channel gluons do not cross. For example, the sum in the last but one term in the equation (2.18) for the four-gluon amplitude extends over the four convolutions

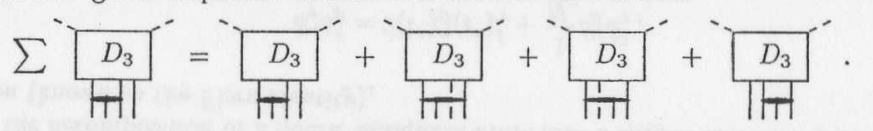

We will now in turn discuss the elements occurring in the integral equations: the inhomogeneous terms $D_{(n ; 0)}$ representing the coupling of $n$ gluons to a quark loop and the integral kernels $K_{2 \rightarrow n}$. But before doing so, we first have to consider some color algebra.

\subsection{Color structure}

In this section we collect some essential facts about color algebra. While doing so we also introduce the so-called birdtrack notation ${ }^{2}$ for structure constants. This diagrammatic notation is extremely useful for the problem of contracting indices of arbitrary color tensors. Such a powerful tool is highly welcome here since tensor contractions constitute an essential part of the computations necessary in the study of the integral equations. The diagrammatic method that serves this purpose is described in detail in appendix A.

We are interested in the structure of the gauge group $G=\mathrm{SU}\left(N_{c}\right)$ with generators $t^{a}$ $\left(a=1, \ldots, N_{c}^{2}-1\right)$ in the Lie algebra su $\left(N_{c}\right)$. The algebra of generators is

$$
\left[t^{a}, t^{b}\right]=i f_{a b c} t^{c} .
$$

For the case of su(3) the $t^{a}$ are given by the well-known Gell-Mann matrices $\lambda^{a}, t^{a}=\lambda^{a} / 2$. The antisymmetric structure constants $f_{a b c}$ can be expressed in terms of generators as

$$
f_{a b c}=-f_{a c b}=-2 i\left[\operatorname{tr}\left(t^{a} t^{b} t^{c}\right)-\operatorname{tr}\left(t^{c} t^{b} t^{a}\right)\right],
$$

diagrammatically

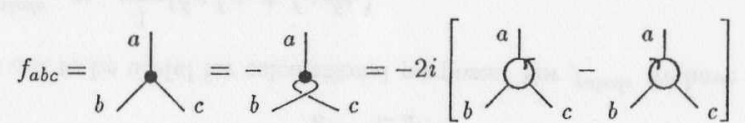

The thicker oriented lines stand for quark color representations, the unorientated lines correspond to gluon color lines. The $f_{a b c}$ are obviously invariant under cyclic permutations of the indices. Normalization of generators is such that for the Killing form

$$
\operatorname{tr}\left(t^{a} t^{b}\right)=\frac{1}{2} \delta_{a b} \quad \text { or } \quad a-{ }_{b}=\frac{1}{2} \bar{b}_{b} .
$$

Using birdtrack notation the algebra (2.22) becomes

$$
\overrightarrow{T_{a}}-\overrightarrow{\rangle_{b}}=i \overrightarrow{\boldsymbol{\lambda}_{b}}
$$

The anticommutator of two generators is

$$
\left\{t^{a}, t^{b}\right\}=\frac{1}{N_{c}} \delta_{a b}+d_{a b c} t^{c},
$$

and the symmetric structure constants $d_{a b c}$ are expressed in terms of generators as

$$
d_{a b c}=d_{a c b}=2\left[\operatorname{tr}\left(t^{a} t^{b} t^{c}\right)+\operatorname{tr}\left(t^{c} t^{b} t^{a}\right)\right],
$$

in diagrams

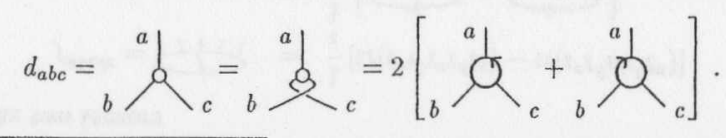

${ }^{2} \mathrm{~A}$ more complete account of this notation can be found in [55] where it is also applied to other Lie groups. Our normalization convention slightly deviates from [55]. 
With this we have collected all basic elements of the birdtrack notation. For later use we give the decomposition of a quark-antiquark state into a singlet and an adjoint representation (known as the Fierz identity),

$$
\delta_{\gamma}^{\alpha} \delta_{\beta}^{\delta}=2\left(t^{a}\right)_{\beta}^{\alpha}\left(t^{a}\right)_{\gamma}^{\delta}+\frac{1}{N_{c}} \delta_{\beta}^{\alpha} \delta_{\gamma}^{\delta},
$$

where $\alpha_{1} \ldots, \delta$ are color labels in the quark (fundamental) representation. In birdtracks it becomes

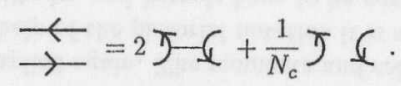

We will slightly extend the birdtrack notation by the definition

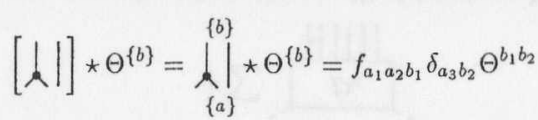

for the contraction of the set of color labels $\{b\}$ of an arbitrary color tensor $\Theta^{\{b\}}$. The symbol $\star$ stands for the contraction of the set $\{b\}$ in color space. The extension to more symbol $\star$ stands for the contraction of the set $\{b\}$
than two elements in the set $\{b\}$ is straightforward.

Generalizing (2.23) and (2.28) we define further tensors that are built from traces of generators in the following way ${ }^{3}$

$$
\begin{aligned}
d^{b_{1} b_{2} \ldots b_{n}} & =\operatorname{tr}\left(t^{b_{1}} t^{b_{2}} \ldots t^{b_{n}}\right)+\operatorname{tr}\left(t^{b_{n}} \ldots t^{b_{2}} t^{b_{1}}\right) \\
f^{b_{1} b_{2} \ldots b_{n}} & =\frac{1}{i}\left[\operatorname{tr}\left(t^{b_{1}} t^{b_{2}} \ldots t^{b_{n}}\right)-\operatorname{tr}\left(t^{b_{n}} \ldots t^{b_{2}} t^{b_{1}}\right)\right] .
\end{aligned}
$$

The definitions are valid for any $n \in \mathbb{N}$. For $n=2,3$, however, the tensors arising from (2.33), (2.34) are proportional ${ }^{4}$ to $\delta_{b_{1} b_{2}}, f_{b_{1} b_{2} b_{3}}$, and $d_{b_{1} b_{2} b_{3}}$ respectively. For the cases $n=2,3$ we will stick to the conventional definitions of structure constants given earlier in $(2.23),(2.28)$. To avoid confusion, the notation with upper indices will be used only in section 5.2 where the most general case will be needed.

The following three tensors are special cases of (2.33), (2.34). We will make extensive use of them throughout this thesis. The tensor

$$
\begin{aligned}
d^{a b c d}=\overparen{T T}_{T} & =\operatorname{tr}\left(t^{a} t^{b} t^{c} t^{d}\right)+\operatorname{tr}\left(t^{d} t^{c} t^{b} t^{a}\right) \\
& =G_{T}+\overbrace{T}
\end{aligned}
$$

${ }^{3}$ In appendix A we will for brevity refer to tensors built from traces over generators in this way as 'standard tensors'. To the best of our knowledge this term does not carry a fixed meaning in the literature on Lie algebras.

${ }^{4}$ In detail we have according to (2.25), (2.28), and (2.23)

$$
\begin{aligned}
d^{a b} & =\delta_{a b} \\
d^{a b c} & =\frac{1}{2} d_{a b c} \\
f^{a b c} & =\frac{1}{2} f_{a b c} .
\end{aligned}
$$

Obviously, one has to be very careful here in distinguishing between upper and lower indices. has been used already in [22] for the investigation of the four-gluon amplitude. We now add to this the two tensors

$$
\begin{aligned}
f^{a b c d e}=\text { TTR } & =\frac{1}{i}\left[\operatorname{tr}\left(t^{a} t^{b} t^{c} t^{d} t^{e}\right)-\operatorname{tr}\left(t^{e} t^{d} t^{c} t^{b} t^{a}\right)\right] \\
& =\frac{1}{i}[\text { ITR - TTR }]
\end{aligned}
$$

and

$$
d^{a b c d e f}=\operatorname{tr}\left(t^{a} t^{b} t^{c} t^{d} t^{e} t^{f}\right)+\operatorname{tr}\left(t^{f} t^{e} t^{d} t^{c} t^{b} t^{a}\right) .
$$

All three are invariant under cyclic permutations of the color labels (as are obviously al tensors defined according to (2.33), (2.34)). It will turn out that these color tensors are very well suited for the whole problem of solving the integral equations. When interpreting the results in terms of reggeon color representations, the decomposition of these tensors into the lower order tensors $f_{a b c}, d_{a b c}$ and $\delta_{a b}$ is also useful:

$$
\begin{aligned}
d^{a b c d} & =\frac{1}{2 N_{c}} \delta_{a b} \delta_{c d}+\frac{1}{4}\left(d_{a b k} d_{k c d}-f_{a b k} f_{k c d}\right) \\
& =\frac{1}{2 N_{c}} \cap \cap+\frac{1}{4}(R \Re-\wedge \wedge),
\end{aligned}
$$

as is easily derived using (2.22) and (2.27). From this we get by cyclic permutation

$$
d^{a b c d}=\frac{1}{2 N_{c}} \delta_{a d} \delta_{b c}+\frac{1}{4}\left(d_{a d k} d_{k b c}+f_{a d k} f_{k b c}\right) .
$$

We further mention the property

$$
d^{b a c d}=d^{a b d c}
$$

which turns out to be useful for calculational purposes. For $f^{a b c d e}$ we have

$$
\begin{aligned}
& f^{a b c d e}=\frac{1}{4 N_{c}}\left(\delta_{a b} f_{c d e}+f_{a b c} \delta_{d e}\right) \\
& +\frac{1}{8}\left(f_{a b k} d_{k c l} d_{l d e}+d_{a b k} f_{k c l} d_{l d e}+d_{a b k} d_{k c l} f_{l d e}-f_{a b k} f_{k c l} f_{l d e}\right) \\
& =\frac{1}{4 N_{c}}(n \Lambda+\Lambda \cap) \\
& +\frac{1}{8}(\Lambda+R+R \cdot R+R+x-\Lambda \cdot \Lambda)
\end{aligned}
$$

and further identities can be obtained from (2.47) by making use of the invariance under cyclic permutations. The tensor $d^{a b c d e f}$ can be decomposed in a similar way,

$$
\begin{aligned}
d^{a b c d e f}= & \frac{1}{4 N_{c}^{2}} \delta_{a b} \delta_{c d} \delta_{e f} \\
& +\frac{1}{8 N_{c}}\left(\delta_{a b} d_{c d k} d_{k e f}-\delta_{a b} f_{c d k} f_{k e f}+d_{a b k} d_{k c d} \delta_{e f}-f_{a b k} f_{k c d} \delta_{e f}\right) \\
& +\frac{1}{16}\left(d_{a b k} d_{k c l} d_{l d m} d_{m e f}-d_{a b k} d_{k c l} f_{l d m} f_{m e J}-d_{a b k} f_{k c l} d_{l d m} f_{m e f}\right. \\
& \quad-d_{a b k} f_{k c l} f_{l d m} d_{m e f}-f_{a b k} d_{k c l} d_{l d m} f_{m e f}-f_{a b k} d_{k c l} f_{l d m} d_{m e f} \\
& \left.\quad-f_{a b k} f_{k c l} d_{l d m} d_{m e f}+f_{a b k} f_{k c l} f_{l d m} f_{m e f}\right) .
\end{aligned}
$$


In birdtracks it becomes

$$
\begin{aligned}
& d^{a b c d e f}=\frac{1}{4 N_{c}^{2}} \cap \cap \cap \\
& +\frac{1}{8 N_{c}}(n R R-n \wedge \wedge+R R n-\wedge \wedge n) \\
& +\frac{1}{16} \text { (Ri-iR - Ri }
\end{aligned}
$$

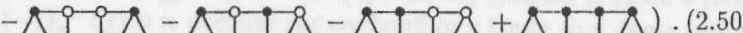

and again other possible decompositions of $d^{a b c d e f}$ are obtained from this by cyclic permutations.

To conclude this section on color algebra, we add a remark on invariant tensors. The gluons of the amplitudes $D_{n}$ form an overall color-singlet. They can therefore be expanded in color space into a linear combination of invariant su $\left(N_{c}\right)$ tensors. Invariant tensors in a simple Lie algebra can generally be constructed from traces of group generators. Consider a tensor

$$
\Theta^{a_{1} \ldots a_{n}}=\operatorname{tr}\left(t^{a_{1}} \ldots t^{a_{n}}\right) .
$$

This trace is obviously invariant under the action of a Lie group element $U \in G$ on the gen erators

$$
t^{a} \longmapsto U t^{a} U^{-1}=U_{A} t^{a}=U_{A}^{a b} t^{b},
$$

where $U_{A}$ is (by definition) the group element $U$ in the adjoint representation. The invariance of the tensor $\Theta$ therefore leads to

$$
\Theta^{a_{1} \ldots a_{n}}=U_{A}^{a_{1} b_{1}} \ldots U_{A}^{a_{n} b_{n}} \operatorname{tr}\left(t^{b_{1}} \ldots t^{b_{n}}\right) .
$$

Let us choose the group element $U$ to correspond to a generator $t^{c}$ via the exponentia map. On the Lie algebra the adjoint representation then acts as the commutator $\left[t^{c}, \cdot\right]$ Therefore the infinitesimal version of (2.53) leads to the condition

$$
\sum_{i=1}^{n} f_{c a_{i} b} \operatorname{tr}\left(t^{a_{1}} \ldots t^{b} \ldots t^{a_{n}}\right)=0,
$$

$t^{b}$ being inserted at the $i$ th position in the trace. Equation (2.54) is often used as the defining property of an invariant tensor. Our tensors $\delta_{a b}, f_{a b c}, d_{a b c}, d^{a b c d}$ etc. are obtained as sum or difference of traces of the form (2.51) and thus invariant tensors fulfilling the condition (2.54). The same holds for products of invariant tensors like $\delta_{a b} f_{c d e}$. For the structure constants $f_{a b d}$, the invariance condition (2.54) results in the well-known Jacobi identity

$$
f_{a b k} f_{k c d}+f_{a c k} f_{b k d}+f_{a d k} f_{k b c}=0 .
$$

\subsection{The quark loop}

Let us now consider the inhomogeneous terms in the integral equations (2.11)-(2.15). The terms $D_{(n ; 0)}^{a_{1} \ldots a_{n}}$ describe the lowest order coupling of $n$ gluons to the quark loop. $D_{(2 ; 0)}^{a_{1} a_{2}}$ is the sum of four cut diagrams

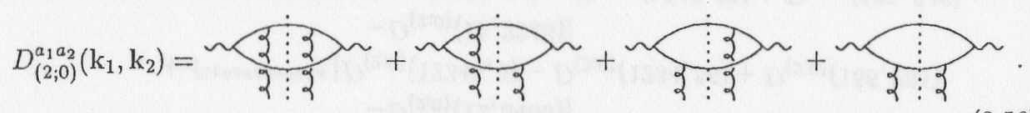

The two gluons are attached to the quark loop in all possible ways to preserve gauge invariance. The $s$-channel cut (indicated in each diagram by the dotted vertical line) implies that the cut quark lines are set on-shell. $D_{(2 ; 0)}^{a_{1} a_{2}}$ depends on the transverse momenta of the two gluons and on their color indices. The latter dependence is of course trivial and we can define the momentum part $D_{(2 ; 0)}\left(\mathrm{k}_{1}, \mathrm{k}_{2}\right)$ of this amplitude by

$$
D_{(2 ; 0)}^{a_{1} a_{2}}\left(\mathrm{k}_{1}, \mathrm{k}_{2}\right)=\delta_{a_{1} a_{2}} D_{(2 ; 0)}\left(\mathrm{k}_{1}, \mathrm{k}_{2}\right) .
$$

An analytic expression for $D_{(2 ; 0)}\left(\mathbf{k}_{1}, \mathbf{k}_{2}\right)$ was first found in [56]. Here we give only a specia case, namely the formula for transversely polarized photons and for the forward direction $\mathbf{k}_{1}=\mathbf{k}_{2}$. It has the form

$$
D_{(2 ; 0)}(l)=\sum_{f} e_{f}^{2} \frac{\alpha_{s}}{2 \pi} \int_{0}^{1} d \alpha \int_{0}^{1} d y \frac{[1-2 \alpha(1-\alpha)][1-2 y(1-y)] l^{2}}{y(1-y) l^{2}+\alpha(1-\alpha)}
$$

where we have replaced the momentum $\mathrm{k}_{1}$ by the momentum $l$ measured in units of $\sqrt{Q^{2}}$ $Q^{2}$ being the squared invariant mass of the photon. The sum extends over the light flavors of quarks which we have assumed to be massless. For longitudinally polarized photon a similar formula can be obtained. The explicit formula for $D_{(2,0)}$ will not be used in the following. We will only need the fact that in general $D_{(2 ; 0)}$ is symmetric under the exchange of its transverse momentum arguments

$$
D_{(2 ; 0)}\left(\mathrm{k}_{1}, \mathrm{k}_{2}\right)=D_{(2 ; 0)}\left(\mathrm{k}_{2}, \mathrm{k}_{1}\right)
$$

and vanishes whenever one of its two arguments vanishes,

$$
\left.D_{(2 ; 0)}\left(\mathbf{k}_{1}, \mathbf{k}_{2}\right)\right|_{\mathbf{k}_{1}=0}=\left.D_{(2 ; 0)}\left(\mathbf{k}_{1}, \mathbf{k}_{2}\right)\right|_{\mathbf{k}_{2}=0}=0 .
$$

In addition, it will be important for the consistency of the integral equations that

$$
D_{(2 ; 0)}\left(\mathrm{k}_{1}, \mathrm{k}_{2}\right)<\text { const. } \log \mathrm{k}_{i}^{2}
$$

in the ultraviolet region, i. e. the growth with the momenta is not stronger than logarithmic

For the amplitudes $D_{(n ; 0)}$ with $n>2$ we again have to attach the $n$ gluons to the quark loop in all possible ways to preserve gauge invariance. But the fact that we are dealing with multiply-cut amplitudes reduces the number of diagrams to consider. The cuts forbid the crossing of $t$-channel gluons as indicated in figure 2.1. The ordering of the gluons along the loop is thus fixed up to the possibility of coupling the gluons to the quark or the antiquark. Therefore we have $2^{n}$ cut diagrams for the inhomogeneous term $D_{(n ; 0)}$.

It turns out that all the amplitudes $D_{(n ; 0)}^{a_{1} \ldots a_{n}}$ can be expressed in terms of $D_{(2 ; 0)}$, the momentum part of $D_{(2 ; 0)}^{a_{1} a_{2}}$ as defined in (2.57). To see how this reduction mechanism works let us have a look at two neighbouring gluons along the quark loop. (They are not necessarily neighbouring as arguments of the amplitude, c.f. gluons 1 and 3 in figure 2.1.) 


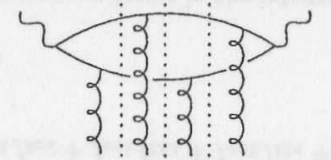

Figure 2.1: Cut amplitude contributing to the coupling of $n=4$ gluons to a quark loop

In the high energy limit the quark-gluon vertices have to be contracted with a longitudinal momentum $p$. The Dirac trace over the quark loop then contains

$$
\frac{{ }^{*}{ }^{*}}{\mathbf{k}_{i}}{ }_{\mathbf{k}_{j}} \simeq \operatorname{tr}(\ldots \not p k p \ldots) \delta\left(k^{2}\right) .
$$

The $\delta\left(k^{2}\right)$ comes in since the quark has to be set on-shell. Using a Sudakov decomposition $k=\alpha q^{\prime}+\beta p+k_{t}$ with $q^{\prime}=q+x p, q^{\prime 2}=p^{2}=0,2 p \cdot q=s$, and $k_{t}^{2}=-\mathrm{k}^{2}$ one finds for this expression

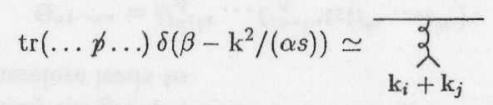

This means that due to energy-momentum conservation only the sum $k_{i}+k_{j}$ of the two momenta enters. We can apply this to all gluons along the quark loop and thereby reduce the momentum part of each diagram to one corresponding to a diagram in which only two gluons are coupled to the quark loop. The color structure is not affected by this reduction. A remark is in order concerning the contribution in which all gluons are coupled to the A remark is in order concerning the contribution in which all gluons are coupled to the quark line or the antiquark line. This term acts as a regularization term. As we will see
below it can be added and subtracted in such a way that the full $D_{(n ; 0)}^{a_{n} \ldots a_{n}}$ can be expressed in terms of $D_{(2 ; 0)}$.

Let us now see how the color structure of the quark loop amplitudes builds up. Obviously, each diagram contributing to $D_{(n ; 0)}$ contains a trace over $n \mathrm{su}\left(N_{c}\right)$ generators. The $2^{n}$ diagrams come in pairs in the following sense. Consider a diagram with $k$ gluons coupled to the quark and $n-k$ gluons coupled to the antiquark. Then there is also a diagram with the $k$ gluons coupled to the antiquark instead of the quark and the other $n-k$ gluons now coupled to the quark. The momentum structure of the two diagrams is the same up to a factor $(-1)^{n}$. (This is because the coupling of a gluon to a quark differs from that to an antiquark by a sign.) The color part of the second diagram is again a trace over generators $t^{a_{i}}$, but in the trace they now appear in reversed order compared to the first diagram. Adding the two diagrams one thus finds a color tensor of the kind $d^{a_{1} \ldots d_{n}}$ for an even number $n$ of gluons and of a tensor of the kind $f^{a_{1} \ldots d_{n}}$ for an odd number $n$ of gluons (cf. (2.33), (2.34) for the definition of the $d$ - and $f$-tensors). There are $2^{n-1}$ pairs of such diagrams. Having in mind that due to the photons at the two ends of the loop the color tersor is not atered if the fist or lop the color ter is
The color structures for two and three gluons attached to the quark loop are more or less trivial: in both cases there is only one color tensor $\left(\delta_{a_{1} a_{2}}\right.$ and $f_{a_{1} a_{2} a_{3}}$, respectively).

We have seen that the diagrams contributing to the quark loop come in $2^{n-1}$ pairs. Among them there is one pair that is special. This is just the pair consisting of the two regularization terms mentioned earlier in which all gluons couple to the quark line or the antiquark line. A closer look shows that this last pair can be added and subtracted with different color structures in such a way that the remaining $2^{n-1}-1$ pairs are regularized to give a $D_{(2 ; 0)}$ each. Therefore the quark loop $D_{(n ; 0)}^{a_{1} \ldots a_{n}}$ can be expressed as a sum of $2^{n-1}-1$ amplitudes $D_{(2 ; 0)}$.

In this thesis, we will need the expressions for the quark loop with up to six gluons attached. As explained, all of them can be written as the sums of $D_{(2 ; 0)}$ amplitudes. For three gluons coupled to the quark loop we find

$$
D_{(3 ; 0)}^{a_{1} a_{2} a_{3}}\left(\mathrm{k}_{1}, \mathrm{k}_{2}, \mathrm{k}_{3}\right)=\frac{1}{2} g f_{a_{1} a_{2} a_{3}}\left[D_{(2 ; 0)}(12,3)-D_{(2 ; 0)}(13,2)+D_{(2 ; 0)}(1,23)\right],
$$

where we use the notation introduced in (2.10). In the case of four gluons the amplitude contains two different color structures,

$$
\begin{gathered}
D_{(4 ; 0)}^{a_{1} a_{2} a_{3} a_{4}}\left(\mathbf{k}_{1}, \mathbf{k}_{2}, \mathbf{k}_{3}, \mathbf{k}_{4}\right)= \\
=-g^{2} d^{a_{1} a_{2} a_{3} a_{4}}\left[D_{(2 ; 0)}(123,4)+D_{(2 ; 0)}(1,234)-D_{(2 ; 0)}(14,23)\right] \\
-g^{2} d^{a_{2} a_{1} a_{3} a_{4}}\left[D_{(2 ; 0)}(134,2)+D_{(2 ; 0)}(124,3)-D_{(2 ; 0)}(12,34)\right. \\
\left.-D_{(2 ; 0)}(13,24)\right] .
\end{gathered}
$$

When five gluons are coupled to the quark loop there appear four different color structures in the corresponding amplitude,

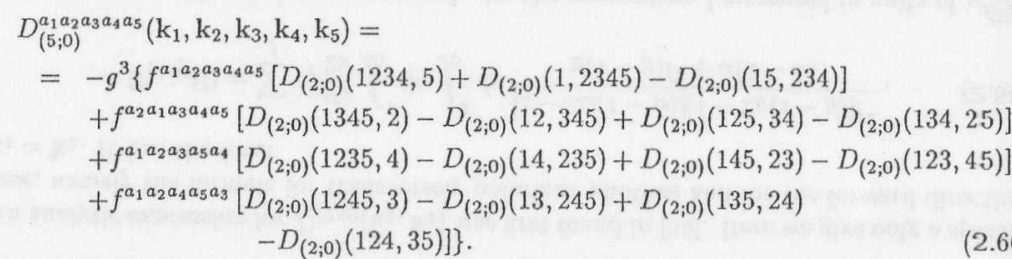

For six gluons attached to the quark loop we find the following expression. Now eight different color structures contribute,

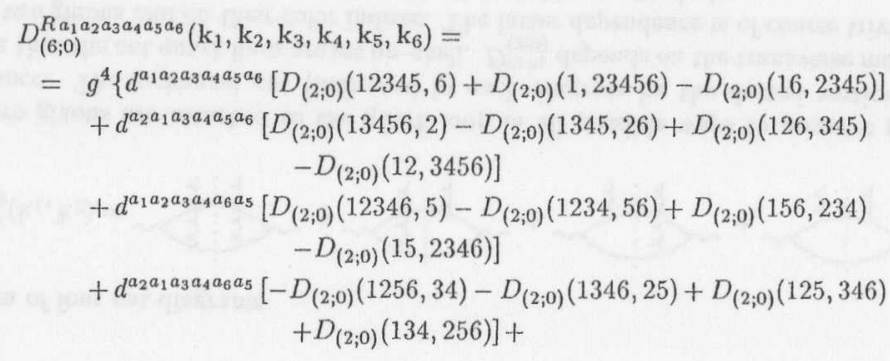


$+d^{a_{3} a_{1} a_{2} a_{4} a_{5} a_{6}}\left[D_{(2 ; 0)}(12456,3)-D_{(2 ; 0)}(1245,36)+D_{(2 ; 0)}(136,245)\right.$ $\left.-D_{(2 ; 0)}(13,2456)\right]$

$+d^{a_{1} a_{2} a_{3} a_{5} a_{6} a_{4}}\left[D_{(2 ; 0)}(12356,4)-D_{(2 ; 0)}(1235,46)+D_{(2 ; 0)}(146,235)\right.$

$\left.-D_{(2 ; 0)}(14,2356)\right]$

$+d^{a_{2} a_{1} a_{3} a_{5} a_{6} a_{4}}\left[-D_{(2 ; 0)}(1246,35)-D_{(2 ; 0)}(1356,24)+D_{(2 ; 0)}(124,356)\right.$ $\left.+D_{(2 ; 0)}(135,246)\right]$

$+d^{a_{1} a_{2} a_{3} a_{6} a_{5} a_{4}}\left[-D_{(2 ; 0)}(1236,45)-D_{(2 ; 0)}(1456,23)+D_{(2 ; 0)}(123,456)\right.$ $\left.\left.+D_{(2 ; 0)}(145,236)\right]\right\}$.

\subsection{Reggeon momentum diagrams}

We now introduce a further diagrammatic notation for the momentum space integrals occurring in our analysis of the integral equations. It will be applied in the next section where we will present the integral kernels $K_{2 \rightarrow m}^{\{b\}} \rightarrow\{a\}$. With the help of so-called reggeon momentum diagrams we hope to make our results more transparent and easier to read. A reggeon momentum integral looks like the following example:

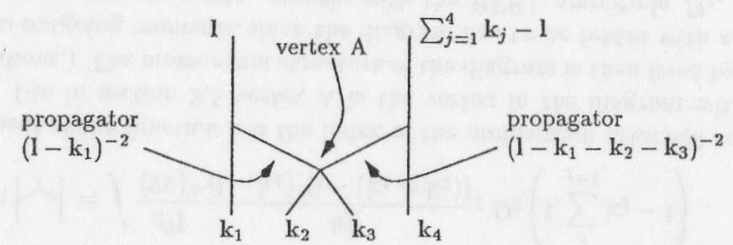

We now state the rules for translating these diagrams back to explicit integrals. At the same time we apply the rules step by step to the above example.

(i) Assign momentum variables to all lines according to momentum conservation (see diagram).

(ii) Write down an integral $\int \frac{d^{2} 1}{(2 \pi)^{3}}$ over the loop momentum. (There is always a loop since some amplitude, for instance $D_{2}$, has to be attached to the upper two lines and thus be written under the integral.)

(iii) Find in the diagram the vertex which has two lines attached from above (vertex A in (2.68)).

(iv) Write down the square of the sum of the momenta attached to this vertex from below. (In our example $\left(\mathrm{k}_{2}+\mathrm{k}_{3}\right)^{2}$.)

(v) Write down propagators for the two lines attached to this vertex from above. (In our example $\left(1-k_{1}\right)^{-2}\left(1-k_{1}-k_{2}-k_{3}\right)^{-2}$.)

For the digram (2.68) this results in

$$
|X|=\int \frac{d^{2} l}{(2 \pi)^{3}} \frac{\left(k_{2}+k_{3}\right)^{2}}{\left(1-k_{1}\right)^{2}\left(1-k_{1}-k_{2}-k_{3}\right)^{2}} .
$$

These rules can easily be inverted in order to construct the reggeon momentum diagram from a given momentum space integral. The reggeon momentum diagrams have to be understood as integral operators. The integration has to be carried out with a function of the two upper momenta. We emphasize that our notation implies only two propagators for a given reggeon momentum diagram.

A few more examples of the diagrammatic notation for momentum space integrals are contained in section 2.7

\subsection{Integral kernels}

The integral kernels $K_{2 \rightarrow m}^{\{b\} \rightarrow\{a\}}$ were calculated in [18] by means of $s$-channel dispersion relations. As explained in section 2.2 the kernels are convoluted with different amplitudes in the integral equations. Only two of the gluons in the respective amplitude actually interact with each other. The kernel acts trivially on the momenta and color labels of the other gluons. We will therefore discuss only the non-trivial action of the kernel here.

other gluons. We will therefore discuss only the non-trivial action of the kernel here.
The kernel for the transition from two gluons with transverse momenta $\mathbf{q}_{1}, \mathbf{q}_{2}$ and color labels $\{b\}=\left\{b_{1}, b_{2}\right\}$ to $m$ gluons with transverse momenta $\mathbf{k}_{1}, \ldots, \mathbf{k}_{m}$ and color labels $\{a\}=\left\{a_{1}, \ldots, a_{m}\right\}$ is given by the general formula

$$
\begin{array}{r}
K_{2 \rightarrow m}^{\{b\} \rightarrow\{a\}}\left(\mathbf{q}_{1}, \mathbf{q}_{2} ; \mathbf{k}_{1}, \ldots, \mathbf{k}_{m}\right)=\prod_{\mathbf{k}_{1}, a_{1}}^{\mathbf{q}_{1}, b_{1}} \prod_{\mathbf{k}_{m}, a_{m}}^{\mathbf{q}_{2}, b_{2}} \\
=f_{b_{1} a_{1} c_{1}} f_{c_{1} a_{2} c_{2}} \ldots f_{c_{m-1} a_{m} b_{2}} g^{m}\left[\left(\mathbf{k}_{1}+\ldots+\mathbf{k}_{m}\right)^{2}-\frac{\mathbf{q}_{2}^{2}\left(\mathbf{k}_{1}+\ldots+\mathbf{k}_{m-1}\right)^{2}}{\left(\mathbf{k}_{m}-\mathbf{q}_{2}\right)^{2}}\right. \\
\left.-\frac{\mathbf{q}_{1}^{2}\left(\mathbf{k}_{2}+\ldots+\mathbf{k}_{m}\right)^{2}}{\left(\mathbf{k}_{1}-\mathbf{q}_{1}\right)^{2}}+\frac{\mathbf{q}_{1}^{2} \mathbf{q}_{2}^{2}\left(\mathbf{k}_{2}+\ldots+\mathbf{k}_{m-1}\right)^{2}}{\left(\mathbf{k}_{1}-\mathbf{q}_{1}\right)^{2}\left(\mathbf{k}_{m}-\mathbf{q}_{2}\right)^{2}}\right] .
\end{array}
$$

For the kernels that are needed for up to six gluons in the $t$-channel this means in our diagrammatic notation for color tensors and momentum integral kernels

$$
\begin{aligned}
& K_{2 \rightarrow 2}^{\{b\} \rightarrow\{a\}}\left(\mathbf{q}_{1}, \mathbf{q}_{2} ;\left\{\mathrm{k}_{i}\right\}\right)=H g^{2} \mathbf{q}_{1}^{2} \mathbf{q}_{2}^{2}[X-M-N] \\
& K_{2 \rightarrow 3}^{\{b\} \rightarrow\{a\}}\left(\mathrm{q}_{1}, \mathrm{q}_{2} ;\left\{\mathrm{k}_{i}\right\}\right)=\not \rightarrow g^{3} \mathrm{q}_{1}^{2} \mathrm{q}_{2}^{2}[X-K|N+K| \\
& K_{2 \rightarrow 4}^{\{b\} \rightarrow\{a\}}\left(q_{1}, q_{2} ;\left\{k_{i}\right\}\right)=\left\lfloor\log g^{4} q_{1}^{2} q_{2}^{2}[X-K|N+| X \mid]\right.
\end{aligned}
$$

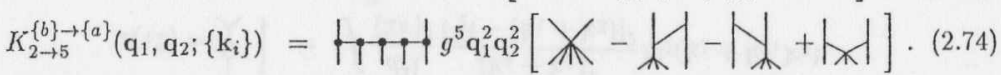

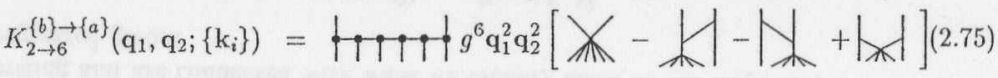

Here the inverse propagators $q_{1}^{2} q_{2}^{2}$ are required in order to cancel the propagators implied by our graphical notation. This is necessary because the kernels $K_{2 \rightarrow m}^{\{b\} \rightarrow\{a\}}$ in the integral equations are defined without propagators; there the propagators come in via the convolution denoted by the symbol $\otimes$. 
We emphasize that the kernel $K_{2 \rightarrow 2}^{\{b\} \rightarrow\{a\}}$ is not identical to the full BFKL kernel (1.9) as it does not contain the trajectory functions $\beta$. In equation (2.11) they have been moved to the left-hand side to make the generalization of this equation to $n>2$ more transparent. Also the kernels $K_{2 \rightarrow n}^{\{b\} \rightarrow\{a\}}$ for $n>2$ do not contain any trajectories and are not infrared finite. It is only in the full integral equations that the divergences cancel.

\subsection{Standard integrals}

When convoluting the amplitudes $D_{n}$ with the kernels $K_{2 \rightarrow m}$ according to the integral equations, we have to deal with a large number of momentum space integrals. It turns out that all these integrals can be classified by a small number of standard integrals. These five standard types of integrals are even sufficient for addressing the case of $n$-gluon amplitudes for any value of $n$. (Strictly speaking this applies to the so-called reggeizing pieces of the amplitudes as will be explained in the next section.) We will therefore give pieces of the amplitudes as will be explained in the next section.) We will therefore give
complete list of the five different standard integrals (or diagrams) which at the same time erve as a few more examples of our notation.

All five standard integrals occur already when dealing with three outgoing gluons. We therefore give the list for $n=3$ here, the generalization to larger $n$ being obvious. First we have

$$
a(2,1)=H=\int \frac{d^{2} \mathrm{l}}{(2 \pi)^{3}} \frac{\mathrm{k}_{2}^{2}}{\left(1-\mathrm{k}_{1}\right)^{2}\left[1-\left(\mathrm{k}_{1}+\mathrm{k}_{2}\right)\right]^{2}} D_{2}\left(1, \sum_{j=1}^{3} \mathrm{k}_{j}-1\right) .
$$

The first argument of the function $a$ is the index of the momentum attached to the vertex A from below. (As in section 2.5 vertex A is the vertex in the diagram with two lines attached from above.) The momentum structure of the diagram is then fixed by giving one of the other two outgoing momenta since the diagram has to be folded with a symmetric function of the upper two momenta, namely with the BFKL amplitude $D_{2}$. We choose the outroing momentum with the lowest index as the second argument of the function (1) contur caried by the third for gong ghon line can easily be infered from the total mumber of gluons. Therefore our notation for $a$ has to be supplied with the total number of $t$-channel gluons it describes. (In the case of more than three gluons we here choose the group of momenta containing the lowest index as the second argument of $a$.) Applying this notation, our earlier example given in (2.68) would be assigned the expression $a(23,1)$ when applied to $\mathrm{D}_{2}$. In section 2.5 the reggeon momentum diagrams stood for integral operators. In this and in the following sections we use the same diagrams also for the convolution with BFKL amplitudes. The meaning should be clear from the context. The function $a$ and the functions $b, c, s, t$ to be defined below always mean the integrals with $D_{2}$ included. The second type of diagram is

$$
b(12)=\left\lfloor=\int \frac{d^{2} \mathrm{l}}{(2 \pi)^{3}} \frac{\left(\mathrm{k}_{1}+\mathrm{k}_{2}\right)^{2}}{\mathbf{l}^{2}\left[1-\left(\mathrm{k}_{1}+\mathrm{k}_{2}\right)\right]^{2}} D_{2}\left(\mathrm{l}, \sum_{j=1}^{3} \mathrm{k}_{j}-1\right) .\right.
$$

The third one is the contact term

$$
c(123)=\chi=\int \frac{d^{2} \mathbf{l}}{(2 \pi)^{3}} \frac{\left(\mathrm{k}_{1}+\mathrm{k}_{2}+\mathrm{k}_{3}\right)^{2}}{1^{2}\left(\mathrm{l}-\sum_{j=1}^{3} \mathrm{k}_{j}\right)^{2}} D_{2}\left(1, \sum_{j=1}^{3} \mathrm{k}_{j}-1\right),
$$

which is local in impact parameter space. The integrals $a, b$, and $c$ correspond to real corrections, that is to $s$-channel gluon production.

Further we have two integral types corresponding to virtual corrections. These are factorizing and are connected with what we already know as the trajectory function $\beta$ of the reggeized gluon:

$$
\begin{aligned}
t(12)=\hat{\curlywedge} \mid & =\int \frac{d^{2} \mathbf{l}}{(2 \pi)^{3}} \frac{\left(\mathrm{k}_{1}+\mathrm{k}_{2}\right)^{2}}{1^{2}\left[1-\left(\mathrm{k}_{1}+\mathrm{k}_{2}\right)\right]^{2}} D_{2}\left(\mathrm{k}_{1}+\mathrm{k}_{2}, \mathrm{k}_{3}\right) \\
& =\frac{2}{N_{c} g^{2}} \beta\left(\mathrm{k}_{1}+\mathrm{k}_{2}\right) D_{2}\left(\mathrm{k}_{1}+\mathrm{k}_{2}, \mathrm{k}_{3}\right)
\end{aligned}
$$

$$
\begin{aligned}
s(1,2) & =\propto \alpha \mid=\int \frac{d^{2} l}{(2 \pi)^{3}} \frac{\mathrm{k}_{1}^{2}}{\mathrm{l}^{2}\left(\mathbf{l}-\mathrm{k}_{1}\right)^{2}} D_{2}\left(\mathrm{k}_{1}+\mathrm{k}_{2}, \mathrm{k}_{3}\right) \\
& =\frac{2}{N_{c} g^{2}} \beta\left(\mathrm{k}_{1}\right) D_{2}\left(\mathrm{k}_{1}+\mathrm{k}_{2}, \mathrm{k}_{3}\right) .
\end{aligned}
$$

This means that the bubble diagrams correspond to gluon trajectory functions $\beta$ for the line they are drawn on.

It suggests itself here to write the BFKL kernel using the standard integrals introduced above. When the kernel is applied to the BFKL amplitude $D_{2}$ we find

$$
\begin{aligned}
K_{\mathrm{BFKL}} \otimes D_{2} & =N_{c} g^{2}\left[c(12)-b(1)-b(2)+\frac{1}{2} t(1)+\frac{1}{2} t(2)\right] \\
& =N_{c} g^{2}\left[\chi-\nvdash-\bigvee+\frac{1}{2} \phi\left|+\frac{1}{2}\right| \phi\right] .
\end{aligned}
$$

The notation introduced here makes implicit use of the fact that the BFKL amplitude $D_{2}$ is symmetric in its two momentum arguments. Without this assumption it would, for example, be necessary to give explicitly a third argument to fully specify the integral a in (2.76). We have chosen to restrict the short-hand notation to the case of a symmetric function since in the remaining chapters we will only apply it to the case of $D_{2}$.

As an example of how the convolutions of $n$-gluon amplitudes with kernels can be reduced to the standard integrals may serve

$D_{2}\left(\mathrm{l}_{1}+\mathrm{l}_{2}, \mathrm{l}_{3}\right) \otimes K_{2 \rightarrow 3}\left(\mathrm{l}_{2}, \mathrm{l}_{3} ; \mathbf{k}_{2}, \mathbf{k}_{3}, \mathbf{k}_{4}\right)=g^{3}[b(234)-a(23,1)-b(34)+a(3,12)] .(2.83)$

This can be easily checked using the explicit definition (2.72) or (2.70) of the kernel. When studying the $n$-gluon amplitudes $D_{n}^{R}$ we encounter a problem connected with these convolutions in momentum space. For each convolution it is rather easy to find a representatio in terms of the standard integrals, as the above example shows. The actual problem is the rapidly increasing number of convolutions we have to deal with when coming to larger $n$. We will explain this problem in more detail in the following chapters. For $n=5 \mathrm{~g}$-luons the problem is at the ede of being tractable by hand. For $n=6$ gluons the problem has introduce the classification of momentum space integrals in this section. In appendix B we give an algorithm suited for implementation on a computer, for instance in the PERL script language. The example above is intended to illustrate that the notation indeed allows to handle the rather complicated integrals in compact form. 
Closing this section, we remark that even the five standard types of integrals mentioned above are not completely independent if we take our definitions (2.76)-(2.80) literally. Relations between them occur in the case that one of the outgoing legs of the diagrams has zero momentum. One finds, for example, that the function b emerges from a when the arguments of $a$ exhaust the outgoing gluons, that is for three gluons we would get

$$
a(1,23)=b(1) .
$$

Similarly, for three outgoing gluons

$$
b(123)=c(123) .
$$

In addition, we find the function $t$ from $s$ when the second argument of $s$ vanishes, for example

$$
s(1,-)=t(1)
$$

In spite of these relations we prefer to treat the five integral types as fundamentally different because they correspond to very different locality properties in impact parameter space. The function $c$, for instance, corresponds to a contact interaction whereas the function $a$ contains a non-locality.

This concludes our discussion of the elements of the integral equations and we can now proceed to solving them (at least partially).

\section{Chapter 3}

\section{Three and Four Gluons, the Transition Vertex $V_{2} \rightarrow 4$}

In this chapter we collect the known results on the three- and four-gluon amplitudes that will be extensively used in the following chapters. At the same time the review of the four-gluon amplitude offers the opportunity to present in this comparatively simple case the method we use for attacking the integral equations. We start with the three-gluor amplitude. The corresponding equation can be solved exactly. We then come to the fourgluon amplitude and explain how the integral equation can be used to gain insight into its structure. The analysis of the four-gluon amplitude leads us in a natural way to the two-to-four gluon vertex $V_{2 \rightarrow 4}$ that plays a prominent role in the analysis of the integra equations also for higher $n$-gluon amplitudes. Its properties are described in some detail. The field theory structure of the unitarity corrections with up to four $t$-channel gluons is discussed. We emphasize that its emergence is closely related to the phenomenon of reggeization. In section 3.4 we explain the conformal invariance of the transition vertex.

Besides the known results the present chapter also contains one new result. In section 3.5 we show that the two-to-four transition vertex $V_{2 \rightarrow 4}$ can be written as a. sum of convolutions of free propagators and BFKL kernels. The corresponding representation is given explicitly and the potential significance of the result is briefly mentioned.

\subsection{The three-gluon amplitude}

The amplitudes with three and four gluons, $D_{3}$ and $D_{4}$ were first investigated in $[21,22]$. It was found that the integral equation (2.12) for the three gluon amplitude can be solved, the solution being

$$
D_{3}^{a_{1} a_{2} a_{3}}\left(\mathbf{k}_{1}, \mathbf{k}_{2}, \mathbf{k}_{3}\right)=\frac{1}{2} g f_{a_{1} a_{2} a_{3}}\left[D_{2}(12,3)-D_{2}(13,2)+D_{2}(1,23)\right],
$$

which can be shown by direct computation. In addition to performing the color algebra contractions and the convolutions in momentum space one has to make use of the integrad equation (2.11) for the two-gluon amplitude $D_{2}$.

The result (3.1) means that an actual three-gluon state does not appear. In the contrary, the amplitude turns out to be a superposition of two-gluon states. We call this phenomenon the reggeization of the amplitude. It generalizes the notion of reggeization 
previously attributed to the fact that the BFKL equation in the color octet channel can be solved by a pole-ansatz and thus describes a one-reggeon state. In the case of the three-gluon amplitude reggeization again occurs in a channel corresponding to the adjoint representation, i.e in the octet channet for the case of $N_{c}=3$. We should emphasize that the reggization of $D$ is a property of the momentum space The analytic properties correspond to those of a superposition of two-gluon compound states. Nevertheless, $D_{3}$ remains a three-gluon amplitude carrying three color labels, i. e. the color part of the amplitude is not affected.

It is worth noting that the solution (3.1) is obtained from the lowest order term (2.64) by replacing the quark loop amplitudes $D_{(2 ; 0)}$ by the full BFKL amplitudes $D_{2}$ while keeping the color and momentum structure.

We did not give a proof of (3.1) here. In chapter 4 we will discover that the reggeization of the three-gluon amplitude $D_{3}$ is actually a special case of a more general identity. The general case will be discussed in detail when the five-gluon amplitude is studied in that chapter.

\subsection{The four-gluon amplitude and the two-to-four transition} vertex $V_{2 \rightarrow 4}$

The method that we use to gain insight into the structure of the $n$-gluon amplitudes is, in principle, very siple. in priciple, very sart of the solution a a new integral equation for the unknown part is derived. So far this is always possible, independent of how good or bad our guess was. But now the new integral equation will tell. If the guess was not very good, the new integral equation will be very complicated and it will not be possible to extract any further information. If the guess was good the new integral equation is simple ${ }^{1}$ and allows to gain further information on the unknown part. Of course, this procedure is not uniquely determined. As we will see, the quark loop is an excellent source of inspiration for the choice of an ansatz. Only in a later step in the investigation of the six - cluon amplitude in chapter 6 we will for the first time have some

for

In $[21,22]$ the four-gluon amplitude was split into two parts,

$$
D_{4}=D_{4}^{R}+D_{4}^{I},
$$

a reggeizing part $D_{4}^{R}$ and a part $D_{4}^{I}$ that for reasons to be explained below is called the irreducible part of the four-gluon amplitude. The reggeizing part is - in analogy to the three-gluon case - chosen as the superposition of two-gluon amplitudes,

$$
\begin{aligned}
D_{4}^{R a_{1} a_{2} a_{3} a_{4}}\left(\mathbf{k}_{1}, \mathbf{k}_{2}, \mathbf{k}_{3}, \mathbf{k}_{4}\right)= \\
=-g^{2} d^{a_{1} a_{2} a_{3} a_{4}}\left[D_{2}(123,4)+D_{2}(1,234)-D_{2}(14,23)\right] \\
\quad-g^{2} d^{a_{2} a_{1} a_{3} a_{4}}\left[D_{2}(134,2)+D_{2}(124,3)-D_{2}(12,34)-D_{2}(13,24)\right] .
\end{aligned}
$$

'The word 'simple' is obviously a critical point. Its exact meaning will hopefully become clear in the course of the present section.
Again, this is obtained from the lowest order term (2.65) by the replacement $D_{(2 ; 0)} \rightarrow D_{2}$ The ansatz for the reggeizing part and thus the decomposition (3.2) is to some extend arbitrary. Recently, a different ansatz for the reggeizing part was investigated in $[57,58]$. That ansatz, as also discussed in [22], is more convenient for the analysis of high mass diffractive processes. We will not further discuss other possible choices for the splitting (3.2) of the amplitude here. All choices will, of course, lead to equivalent results for the (3.2) of the amplitude here. All choices will, of course, lead to equivalent results for the
complete amplitude. The choice given above is singled out because it leads to a very complete amplitude. The choice given above is singled out because it leads to a very
simple picture for the remaining part $D_{4}^{I}$, especially when interpreted in view of a field simple picture for the remaining part $D_{4}^{\prime}$, especially when interpreted in view of a field theory structure of unitarity corrections. Moreover, the

The natural next step is to derive a new integral equation for the unknown irreducible part $D_{4}^{I}$. To this end we insert the ansatz (3.2),(3.3) for the full amplitude into the original integral equation (2.13). The known result (3.1) for the three gluon amplitude is inserted as well. Due to the choce of $D^{R}$ we can then apply the equa as well. Due to the choice of $D^{R}$ we can the (3.1) for the the amplitude to the expression $\omega D_{4}^{R}$ on the left hand side. That exactly eliminates the lowest order term $D_{(4 ; 0)}$ on the right hand side and produces additional terms containing only the
convolution of $D_{2}$ amplitudes with the kernel $K_{2 \rightarrow 2}^{\{b\}}\{a\}$ or products of $D_{2}$ with trajectory convolution of $D_{2}$ amplitudes with the kernel $K_{2 \rightarrow 2}^{\{b\} \rightarrow\{a\}}$ or products of $D_{2}$ with trajectory
functions $\beta$. Besides the terms containing $D_{4}^{I}$ all other contributions to the right hand side consist of convolutions of two-gluon amplitudes $D_{2}$ with the integral kernels. Our new equation thus takes the form

$$
\left(\omega-\sum_{i=1}^{4} \beta\left(\mathrm{k}_{i}\right)\right) D_{4}^{I a_{1} a_{2} a_{3} a_{4}}\left(\mathrm{k}_{1}, \mathrm{k}_{2}, \mathrm{k}_{3}, \mathrm{k}_{4}\right)=V_{2 \rightarrow 4}^{a_{1} a_{2} a_{3} a_{4}} D_{2}+\sum K_{2 \rightarrow 2}^{\{b\} \rightarrow\{a\}} \otimes D_{4}^{I b_{1} b_{2} b_{3} b_{4}} .
$$

The sum on the right-hand side of this new equation extends over all pairwise interaction of the four gluons. In the inhomogeneous term $V_{2 \rightarrow 4} D_{2}$ we collect all the terms containing $D_{2}$, hence the notation. $V_{2 \rightarrow 4}$ should be understood as an integral operator acting on the two-gluon amplitude. As we will explain in more detail below, it describes the transition from the two-gluon state to a fully interacting four-gluon system in the $t$-channel. The explicit expression for the two-to-four transition vertex was computed in [21, 22]. To arrive at this explicit result the following steps have to be done. First we have to con tract the color tensors of the amplitules with those of the beris. The nes described in appendix A. The second step is to bring the momentum spact to their standard form, that is to classify them according to the scheme explained i section 2.7. Both steps result in lengthy calculations because of the large number of contractions involved. The results of the $\mathrm{su}\left(N_{c}\right)$ tensor contractions are then multiplier with the corresponding momentum space integrals. Finally, all terms can be collected to give the vertex $V_{2 \rightarrow 4}$. Due to cancellations the resulting expression is comparatively compact. Remarkably, all terms belonging to the color tensors $d^{a_{1} a_{2} a_{3} a_{4}}$ and $d^{a_{2} a_{1} a_{3} a_{4}}$ drop out. One finds the following color and momentum structure for the vertex:

$$
\begin{aligned}
V_{2 \rightarrow 4}^{a_{1} a_{2} a_{3} a_{4}}\left(\left\{\mathbf{q}_{j}\right\}, \mathbf{k}_{1}, \mathbf{k}_{2}, \mathbf{k}_{3}, \mathbf{k}_{4}\right)= & \delta_{a_{1} a_{2}} \delta_{a_{3} a_{4}} V\left(\left\{\mathbf{q}_{j}\right\}, \mathbf{k}_{1}, \mathbf{k}_{2} ; \mathbf{k}_{3}, \mathbf{k}_{4}\right) \\
& +\delta_{a_{1} a_{3}} \delta_{a_{2} a_{4}} V\left(\left\{\mathbf{q}_{j}\right\}, \mathbf{k}_{1}, \mathrm{k}_{3} ; \mathbf{k}_{2}, \mathbf{k}_{4}\right) \\
& +\delta_{a_{1} a_{4}} \delta_{a_{2} a_{3}} V\left(\left\{\mathbf{q}_{j}\right\}, \mathbf{k}_{1}, \mathbf{k}_{4} ; \mathbf{k}_{2}, \mathbf{k}_{3}\right) .
\end{aligned}
$$

The function $V$ is the same in all three terms on the right hand side. The $\mathbf{q}_{j}$ are the two momenta entering from above. Since throughout this thesis $V_{2 \rightarrow 4}$ is almost al ways 
contracted with a BFKL amplitude $D_{2}$ from above, we will omit these arguments in the following and consider the quantity $V_{2 \rightarrow 4} D_{2}$. The vertex function $V\left(\mathbf{k}_{1}, \mathrm{k}_{2} ; \mathrm{k}_{3}, \mathrm{k}_{4}\right)$ has the explicit form

$$
\begin{aligned}
\left(V D_{2}\right)\left(\mathrm{k}_{1}, \mathrm{k}_{2} ; \mathrm{k}_{3}, \mathrm{k}_{4}\right)=\frac{g^{4}}{4} \times\{2 & {[c(1234)} \\
& -b(123)-b(124)-b(134)-b(234)+b(12)+b(34) \\
& +a(13,2)+a(14,2)+a(23,1)+a(24,1) \\
& -a(1,2)-a(2,1)-a(3,12)-a(4,12)] \\
+ & t(123)+t(124)+t(134)+t(234)-t(12)-t(34) \\
& -s(13,2)-s(13,4)-s(14,2)-s(14,3) \\
& -s(23,1)-s(23,4)-s(24,1)-s(24,3) \\
& +s(1,2)+s(1,34)+s(2,1)+s(2,34) \\
& +s(3,12)+s(3,4)+s(4,12)+s(4,3)]\}
\end{aligned}
$$

where we have made use of the notation introduced in section 2.7 .

Let us now describe the known properties of the vertex function $V$ and of the full transition vertex $V_{2 \rightarrow 4}$. The first observation is that $V\left(\mathrm{k}_{1}, \mathrm{k}_{2} ; \mathrm{k}_{3}, \mathrm{k}_{4}\right)$ is symmetric in its first two and in its last two arguments

$$
\begin{aligned}
V\left(\mathbf{k}_{1}, \mathbf{k}_{2} ; \mathbf{k}_{3}, \mathbf{k}_{4}\right) & =V\left(\mathbf{k}_{2}, \mathrm{k}_{1} ; \mathbf{k}_{3}, \mathbf{k}_{4}\right) \\
& =V\left(\mathbf{k}_{1}, \mathbf{k}_{2} ; \mathbf{k}_{4}, \mathbf{k}_{3}\right),
\end{aligned}
$$

and symmetric under the exchange of the first pair of arguments and the second pair of arguments (that is why our notation separates these pairs by a semicolon)

$$
V\left(\mathbf{k}_{1}, \mathbf{k}_{2} ; \mathbf{k}_{3}, \mathbf{k}_{4}\right)=V\left(\mathbf{k}_{3}, \mathbf{k}_{4} ; \mathbf{k}_{1}, \mathbf{k}_{2}\right) .
$$

Therefore, according to (3.5) the full vertex $V_{2 \rightarrow 4}$ is completely symmetric under the simultaneous exchange of color and momentum of the gluons.

The combination of integrals in $V D_{2}$ vanishes when one of the outgoing momenta vanishes,

$$
\left.\left(V D_{2}\right)\left(\mathrm{k}_{1}, \mathrm{k}_{2} ; \mathrm{k}_{3}, \mathrm{k}_{4}\right)\right|_{\mathrm{k}_{i}=0}=0 \quad(i \in\{1, \ldots, 4\}) .
$$

This result can be proven easily using identities of the kind mentioned at the end of section 2.7 and the fact that the BFKL amplitude $D_{2}$ vanishes at zero-momentum argument. This property of $V D_{2}$ is carried over to the full vertex,

$$
\left.\left(V_{2 \rightarrow 4} D_{2}\right)^{a_{1} a_{2} a_{3} a_{4}}\left(\mathrm{k}_{1}, \mathrm{k}_{2} ; \mathrm{k}_{3}, \mathrm{k}_{4}\right)\right|_{\mathbf{k}_{i}=0}=0 \quad(i \in\{1, \ldots, 4\}) .
$$

Further, the function $V D_{2}$ is infrared finite, that is the infrared divergences in the different integrals contributing to (3.6) cancel in the sum. This can be easily seen after noticing that already certain combination of very few standard integrals are infrared finite. For instance, the combination

$$
b(\mathrm{l})-\frac{1}{2} t(1)
$$

is infrared finite for any sum of momenta that is substituted for 1 . The proof is obvious by inspection of the corresponding integrals (see section 2.7). The factor $1 / 2$ comes about because the integrand of the trajectory function $\beta$ (see (2.79) and (1.10)) exhibits two divergences of the same form. Similarly, one can show that the combination

$$
a\left(\mathbf{l}_{2}, \mathrm{l}_{1}\right)-\frac{1}{2} s\left(\mathrm{l}_{2}, \mathrm{l}_{1}\right)-\frac{1}{2} s\left(\mathrm{l}_{2}, \mathrm{l}_{3}\right)
$$

is infrared finite separately for any partition of the momenta $\left\{\mathrm{k}_{i}\right\}$ into three sums $\mathrm{l}_{1}, \mathrm{l}_{2}, \mathrm{l}_{3}$. Finally, the term $c(1)$ is infrared finite separately since in this term (see (2.78)) the poles of the propagators are cancelled by the zeros of the BFKL amplitude $D_{2}$ in the integral. The finiteness of these three groups is completely independent of the total number of momenta $\mathbf{k}_{i}$ that are split into the groups denoted by $l_{j}$. It should be obvious from equation (3.6) that the integrals in the vertex function come in exactly these infrared finite combinations.

Having discussed some nice properties of the transition vertex $V_{2 \rightarrow 4}$ we now come back to the main problem of understanding the full four-gluon amplitude $D_{4}$. When the ansatz (3.2) was made the goal was to arrive at a simple equation for the yet unknown part $D_{4}^{I}$. A glance at the new integral equation (3.4) tells us that the ansatz inspired by the quark loop was a very good guess. In fact, the equation contains only the vertex $V_{2 \rightarrow 4}$ and a homogeneous part. Therefore, the integral equation (3.4) for $D_{4}^{I}$ can now be iterated. The homogeneous part. Therefore
structure arising from this is

$$
D_{4}^{I}=G_{4} \cdot V_{2+4} \cdot D_{2}
$$

$G_{4}$ being the Green function of the four-gluon state. The Green function obeys the BKP equation with four $t$-channel gluons which is a four-particle Schrödinger equation. Its Hamiltonian is given by the homogeneous part of the integral equation (3.4), i.e. by the sum of all pairwise interactions $K_{2 \rightarrow 2}$ of the four gluons. Unfortunately, the eigenvalues and eigenfunctions of the Hamiltonian are not known and (3.13) remains a formal solution and eigenfunctions of the Hamiltonian are not known and (3.13) remains a formal solution
only. Though, some properties of the four-gluon state have been worked out in [24]. We only. Though, some properties of the four-gluon state have been worked out in [24]. We
will return to the interpretation of the structure inherent in (3.13) momentarily in section 3.3 .

Even without knowing an analytic formula for $D_{4}^{I}$ we can deduce two important properties. Like the two-gluon amplitude, the irreducible part of the four-gluon amplitude vanishes (modulo logarithms) when one of the outgoing gluon momenta is set to zero,

$$
\left.D_{4}^{I a_{1} a_{2} a_{3} a_{4}}\left(\mathbf{k}_{1}, \mathbf{k}_{2}, \mathbf{k}_{3}, \mathbf{k}_{4}\right)\right|_{\mathbf{k}_{i}=0}=0 \quad(i \in\{1, \ldots, 4\}) .
$$

To prove this we proceed order by order in the iteration of the Hamiltonian $\sum K_{2 \rightarrow 2}$. The identity holds in lowest order since the vertex itself has this property (see (3.10)). In the next order, (3.14) holds because $K_{2 \rightarrow 2}$ also vanishes if one of the outgoing momenta becomes zero, etc. Similarly, we can show that the irreducible part $D_{4}^{I}$ is completely symmetric in the four gluons, that is under the simultaneous exchange of color and momentum,

$$
\begin{aligned}
D_{4}^{I a_{1} a_{2} a_{3} a_{4}}\left(\mathbf{k}_{1}, \mathbf{k}_{2}, \mathbf{k}_{3}, \mathrm{k}_{4}\right) & =D_{4}^{I a_{2} a_{1} a_{3} a_{4}}\left(\mathbf{k}_{2}, \mathbf{k}_{1}, \mathbf{k}_{3}, \mathbf{k}_{4}\right) \\
& =D_{4}^{I a_{3} a_{2} a_{1} a_{4}}\left(\mathbf{k}_{3}, \mathbf{k}_{2}, \mathbf{k}_{1}, \mathbf{k}_{4}\right) \\
& =D_{4}^{I a_{4} a_{2} a_{3} a_{1}}\left(\mathbf{k}_{4}, \mathbf{k}_{2}, \mathbf{k}_{3}, \mathbf{k}_{1}\right) .
\end{aligned}
$$

\subsection{Field theory structure}

Although we do not have an analytic expression for the irreducible part, we have gathered by now quite some knowledge about the structure of the four-gluon amplitude $D_{4}$. Ne- 
glecting for a moment color and normalization factors, this structure is illustrated in the following diagram

$$
D_{4}\left(\mathrm{k}_{1}, \mathrm{k}_{2}, \mathrm{k}_{3}, \mathrm{k}_{4}\right)=\sum \sum_{i=1}^{V_{2 \rightarrow 4}}
$$

The first part is the superposition of two-gluon states (the reggeizing part $D_{4}^{R}$ ) coupled to the quark loop. In the second (irreducible) part $D_{4}^{I}$ a two-gluon system couples to the quark loop and then undergoes a transition to a four-gluon compound state via the verte $V_{2 \rightarrow 4}$. From this we learn that the complete amplitude consists of only a few basic building blocks: a quark loop allowing the coupling of the $t$-channel gluons to external particles, the two-gluon Green function (BFKL amplitude), the four-gluon Green function, and the two-to-four transition vertex. The Green functions describe the quantum mechanica propagation of bound states of $t$-channel gluons. With the transition vertex we have in

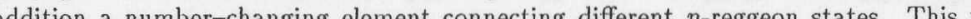
the quantum mechanical probit of a quantum field theory of reggeized gluons. All this happens in the 2-dimensional space of transverse momenta. The complex angular momentum $\omega$ plays the role of an energy variable. Its conjugate variable, i. e. rapidity, plays the role of the time variable.

The structure of unitarity corrections becomes even more interesting when we change from transverse momentum space to impact parameter space by means of a Fourier transform. As was observed in [23] the transition vertex $V_{2 \rightarrow 4}$ in impact parameter space is invariant under conformal transformations. (We will come back to the conformal invariance in some more detail in the next section.) Recalling that also the BFKL amplitude (and in a silar way the four dimensional impact parate the four-glon anplitude exhibit conformal invariance. The occurrence of a field theory structure and of conformal invariance gives rise to the hope that the complete set of unitarity corrections can be cast into the form of an effective field theory. Its basic element should be $n$-gluon Green functions and number changing vertices. It should be a $2+1$ dimensional euclidian field theory. The two space-like dimension are the two dimensions of impact parameter space, the time-like dimension is realized as rapidity. In addition, we expect the effective field theory to be a conformal field theory. The latter property would of course have far-reaching consequences and would allow to apply all the beautifu and powerful methods from conformal field theory. We will briefly mention some of these consequences in the next section.

So far only the simplest elements of a potential effective field theory have been found: the two-gluon compound state, the two-to-four transition vertex $V_{2 \rightarrow 4}$ and the four-gluo compound state. An analytic formula for the latter is still missing. The concept of a effective field theory has not been derived from first principles. It has to be tested and further elements should be derived. To achieve this is seems natural to proceed to highe $n$-gluon amplitudes. Exactly this constitutes the main goal of this thesis, namely to deepen theory structure is the phenomenon of reggeization. Without reggeization in the three gluon amplitude and in the part $D_{4}^{R}$ of the four-gluon amplitude one would not have been able to arrive at the simple structure (3.16). We will therefore pay special attention to the mechanism of reggeization in the unitarity corrections.

\subsection{Conformal invariance in impact parameter space}

The most striking property of the transition vertex $V_{2 \rightarrow 4}$ is its conformal invariance in impact parameter space which was proven in [23]. To explain it in a more detail let us consider an amplitude $\mathcal{A}_{4}$ that is the convolution of the vertex $V_{2 \rightarrow 4}$ with a. BFKL amplitude $\phi_{2}$ from above and with a four-gluon state $\phi_{4}$ from below. The latter is assumed to be a solution of the four-particle BKP equation. The amplitude $\mathcal{A}_{4}$ has the form

$$
\begin{aligned}
\mathcal{A}_{4}= & \int \prod_{j=1}^{2} d^{2} \mathbf{q}_{j} \prod_{i=1}^{4} d^{2} \mathrm{k}_{i} \phi_{2}\left(\mathrm{q}_{1}, \mathbf{q}_{2}\right) V_{2 \rightarrow 4}^{a_{1} a_{2} a_{3} a_{4}}\left(\left\{\mathbf{q}_{j}\right\}, \mathbf{k}_{1}, \mathbf{k}_{2}, \mathrm{k}_{3}, \mathrm{k}_{4}\right) \times \\
& \times \phi_{4}^{a_{1} a_{2} a_{3} a_{4}}\left(\mathrm{k}_{1}, \mathrm{k}_{2}, \mathrm{k}_{3}, \mathrm{k}_{4}\right) \delta\left(\sum_{j=1}^{2} \mathbf{q}_{j}-\sum_{i=1}^{4} \mathrm{k}_{i}\right) .
\end{aligned}
$$

We change from transverse momentum space to impact parameter space by means of a Fourier transform,

$$
\begin{aligned}
\phi_{2}\left(\mathbf{q}_{1}, \mathbf{q}_{2}\right) & =\int \prod_{j=1}^{2}\left[d^{2} \rho_{j^{\prime}} e^{i \mathbf{q} \rho_{j} \rho_{j^{\prime}}}\right] \phi_{2}\left(\rho_{1^{\prime}}, \rho_{2^{\prime}}\right) \\
\phi_{4}\left(\mathbf{k}_{1}, \mathbf{k}_{2}, \mathbf{k}_{3}, \mathbf{k}_{4}\right) & =\int \prod_{i=1}^{4}\left[d^{2} \rho_{i} e^{-i \mathbf{k}_{i} \rho_{i}}\right] \phi_{4}\left(\rho_{1}, \rho_{2}, \rho_{3}, \rho_{4}\right)
\end{aligned}
$$

where the coordinates $\rho$ are understood in complex notation as introduced in section 1.4. This procedure defines the Fourier transform of the transition vertex. Assuming the conformal invariance of the functions $\phi_{2}$ and $\phi_{4}$ one can prove the invariance of the whole amplitude $\mathcal{A}_{4}$ and infer that the vertex in fact is conformally invariant. In this context conformal invariance means the invariance under a simultaneous Möbius transformation of all coordinates $\rho$ according to the map (1.24) in section 1.4. Motivated by the conformal invariance, an operator representation for the transition vertex $V_{2 \rightarrow 4}$ in impact parameter space was worked out in $[24,59]$.

In [23] four subgroups of the integrals in the vertex function (3.6) were found that are infrared finite separately. Their properties under conformal transformations in impact parameter space were then considered separately to eventually prove the invariance of the full vertex. These groups turned out to be suitable also for finding the operator representation for the vertex $[24,59]$. In [57] it was shown that the proof of conformal invariance can be simplified by making use of a representation that can be found al ready in [22] for the forward direction $\left(\sum_{i} \mathbf{k}_{i}=0\right)$,

$$
\begin{aligned}
\left(V D_{2}\right)\left(\mathbf{k}_{1}, \mathbf{k}_{2} ; \mathbf{k}_{3}, \mathbf{k}_{4}\right)=\frac{g^{2}}{2} & {[G(1,23,4)+G(2,13,4)+G(1,24,3)+G(2,14,3)} \\
& -G(12,3,4)-G(12,4,3)-G(1,2,34)-G(2,1,34) \\
& +G(12,-, 34)] .
\end{aligned}
$$


This representation holds for the non-forward direction as well. The function $G$ is

$$
\begin{aligned}
& G\left(\mathbf{k}_{1}, \mathbf{k}_{2}, \mathbf{k}_{3}\right)=\frac{g^{2}}{2}[2 c(123)-2 b(12)-2 b(23)+2 a(2,1) \\
& +t(12)+t(23)-s(2,1)-s(2,3)] \\
& =\frac{g^{2}}{2}[2\rangle-2 \mid\langle-2|\lambda+2| \\
& \left.+\phi|+| \dot{\alpha}-\lambda_{a}|-| d\right] \text {. }
\end{aligned}
$$

After Fourier transformation to configuration space, the function $G$ was shown to be conformally invariant by itself. The conformal invariance of $V_{2 \rightarrow 4}$ can then be derived easily. Therefore it might seem that the function $G$ acquires a fundamental meaning as a generalization of the BFKL kernel for the case of more than two reggeons. $G$ is infrared finite and it vanishes when the first or third argument vanishes, but it does not when the second momentum becomes zero. A direct interpretation of the function $G$ seems difficult since a real two-to-three transition does not occur in the amplitudes. Only the combination (3.20) of the functions $G$ has all the nice properties discussed in section 3.2. We will meet the function $G$ again in chapter 6 where a different combination of $G$ functions will occur. The function $G$ is certainly valuable at least at as a calculational tool.

The conformal invariance might turn out to be a general feature of the unitarity corrections. The present situation seems in fact very promising concerning this issue. In case an effective conforit fold theory of uit ity corrections can be forme an effective cofor and fritul reserch in the past yeas, see for examplo [00, 61]. a subject of very intensive and fruitful research in the past years, see for example $[60,61]$ A result that is immediately relevant to the unitarity corrections is the fact that conforma symmetry imposes very strong constraints on the correlation functions of the theory. An example is the three-point function in a CFT. It is fixed up to a constant for a given set of three conformal fields. In a general CFT there is a set of quasi-primary fields $\Phi(z, \bar{z})$ that transforms under conformal transformations $z \rightarrow f(z)$ as

$$
\Phi(z, \bar{z}) \rightarrow\left(\frac{\partial f}{\partial z}\right)^{h}\left(\frac{\partial \bar{f}}{\partial \bar{z}}\right)^{\bar{h}} \Phi(f(z), \bar{f}(\bar{z})) .
$$

Here the real-valued quantities $h$ and $\bar{h}$ are the conformal weights of the state $\Phi$, and $\bar{h}$ does not indicate the complex conjugate of $h$. (For the relation of the conformal weight to conformal spin and scaling dimension see section 1.4.) The three-point function $G^{(3)}=$ $\left\langle\Phi_{1} \Phi_{2} \Phi_{3}\right\rangle$ has the form

$$
G^{(3)}\left(z_{i}, \bar{z}_{i}\right)=C_{123} \frac{1}{z_{12}^{h_{1}+h_{2}-h_{3}} z_{23}^{h_{2}+h_{3}-h_{1}} z_{13}^{h_{3}+h_{1}-h_{2}}} \cdot \frac{1}{\bar{z}_{12}^{\bar{h}_{1}+\bar{h}_{2}-\bar{h}_{3}} \bar{z}_{23} \bar{h}_{2}+\bar{h}_{3}-\bar{h}_{1} \bar{z}_{13}+\bar{h}_{13}-\bar{h}_{2}},
$$

where $z_{i j}=z_{i}-z_{j}$. Most interestingly, the transition vertex $V_{2 \rightarrow 4}$ has exactly this form when projected onto three BFKL eigenfunctions. In detail this projection is done by choosing the functions $\phi_{2}$ and $\phi_{4}$ in 3.17 as given by conformal eigenfunctions $E^{(\nu)}\left(\rho_{10}, \rho_{20}\right)$,

$$
\phi_{2}\left(\rho_{1^{\prime}}, \rho_{2^{\prime}}\right)=E^{\left(\nu_{c}\right)}\left(\rho_{1^{\prime} c}, \rho_{2^{\prime} c}\right)
$$

$\phi_{4}^{a_{1} a_{2} a_{3} a_{4}}\left(\rho_{1}, \rho_{2}, \rho_{3}, \rho_{4}\right)=\delta_{a_{1} a_{2}} \delta_{a_{3} a_{4}} E^{\left(\nu_{a}\right) *}\left(\rho_{1 a}, \rho_{2 a}\right) E^{\left(\nu_{b}\right) *}\left(\rho_{1 b}, \rho_{2 b}\right)$
This result on the triple Pomeron vertex was found in [24]. The constant in the three point function (which is actually a function of the three conformal weights) was calculated in $[62,63]$. With this impressive result we conclude our short review of the conformal invariance of the two-to-four transition vertex.

\subsection{New representation for the vertex $V_{2 \rightarrow 4}$}

In this section we present a newly discovered property of the vertex $V_{2 \rightarrow 4}$. The new result is of possible relevance to a more conceptional problem, namely the emergence of conformal invariance in the vertex. The vertex $V_{2}$ is a basic (in fact the first number changing) element of the effective field theory. We have made the surprising finding that changing) element of the effective field theory. We have made the surprising finding that it can nevertheless be built entirely from well-known BFKL kernels and free propagators
$\left(1 / \mathrm{k}^{2}\right)$. We do not present the full derivation of the new representation here. The basic idea is to express the kernels $K_{2 \rightarrow m}$ with $m \leq 4$ in terms of two-to-two kernels $K_{2 \rightarrow 2}$. (This was done for the two-to-three kernel $K_{2 \rightarrow 3}$ also in [64] ${ }^{2}$.) Then the color tensors can be shown to arrange in such a way that that the kernel $K_{2 \rightarrow 2}$ can be replaced in these expressions by the full BFKL kernel including the trajectory functions $\beta$. The way the BFKL kernels are convoluted with the propagators in the new representation does not allow to read off the conformal invariance of the vertex immediately. Therfore our new representation is probably not helpful for actual computations. But it is well conceivable that it can contribute to the understanding of the conformal symmetry present in this element of the effective field theory. It might also help to gain insight into the properties of the vertex under crossing. Unfortunately, we are presently not able to reveal the deeper of the vertex under crossing.
meaning of our new result.

meaning of our new result.
To display the formula, we first define $\mathcal{K}$ to be the product of a full BFKL kernel with the two propagators entering from above,

$$
\mathcal{K}\left(\mathbf{q}_{1}, \mathbf{q}_{2} ; \mathbf{k}_{1}, \mathbf{k}_{2}\right)=\frac{-1}{N_{c} g^{2}} \frac{1}{\mathbf{q}_{1}^{2}} \frac{1}{\mathbf{q}_{2}^{2}} K_{\mathrm{BFKL}}\left(\mathbf{q}_{1}, \mathbf{q}_{2} ; \mathbf{k}_{1}, \mathbf{k}_{2}\right),
$$

where (cf. (1.9)) the kernel $K_{\mathrm{BFKL}}$ includes the trajectory functions $\beta$. Using $\mathcal{K}$ the vertex function $V$ (see (3.6)) can then be written as

$$
\begin{aligned}
& \left(V D_{2}\right)\left(\mathbf{k}_{1}, \mathbf{k}_{2} ; \mathbf{k}_{3}, \mathbf{k}_{4}\right)=-\frac{g^{4}}{4(2 \pi)^{3}} \int\left(\prod_{i=1}^{4} d^{2} \mathbf{l}_{i}\right) \delta\left(\sum_{j=1}^{4} \mathbf{l}_{j}-\sum_{j=1}^{4} \mathbf{k}_{j}\right) \\
& \times\left\{\begin{array}{c}
- \\
{\left[D_{2}\left(\mathrm{l}_{1}+\mathrm{l}_{2}+\mathrm{l}_{3}, \mathrm{l}_{4}\right)+D_{2}\left(\mathrm{l}_{1}+\mathrm{l}_{2}+\mathrm{l}_{4}, \mathrm{l}_{3}\right)+D_{2}\left(\mathrm{l}_{1}+\mathrm{l}_{3}+\mathrm{l}_{4}, \mathrm{l}_{2}\right)\right.} \\
+D_{2}\left(\mathrm{l}_{1}, \mathrm{l}_{2}+\mathrm{l}_{3}+\mathrm{l}_{4}\right)-D_{2}\left(\mathrm{l}_{1}+\mathrm{l}_{2}, \mathrm{l}_{3}+\mathrm{l}_{4}\right)-D_{2}\left(\mathrm{l}_{1}+\mathrm{l}_{3}, \mathrm{l}_{2}+\mathrm{l}_{4}\right)
\end{array}\right.
\end{aligned}
$$$$
\left.-D_{2}\left(\mathrm{l}_{1}+\mathrm{l}_{4}, \mathrm{l}_{2}+\mathrm{l}_{3}\right)\right]
$$

$\times\left[\mathcal{K}\left(\mathbf{l}_{1}, \mathbf{l}_{2} ; \mathbf{k}_{1}, \mathbf{k}_{2}\right) \delta\left(\mathbf{l}_{3}-\mathbf{k}_{3}\right) \delta\left(\mathbf{l}_{4}-\mathbf{k}_{4}\right)+\mathcal{K}\left(\mathbf{l}_{3}, \mathbf{l}_{4} ; \mathbf{k}_{3}, \mathbf{k}_{4}\right) \delta\left(\mathbf{l}_{\mathbf{1}}-\mathbf{k}_{1}\right) \delta\left(\mathbf{l}_{2}-\mathbf{k}_{2}\right)\right]$

$+\left[D_{2}\left(\mathrm{l}_{1}+\mathrm{l}_{2}+\mathrm{l}_{3}, \mathrm{l}_{4}\right)+D_{2}\left(\mathrm{l}_{1}, \mathrm{l}_{2}+\mathrm{l}_{3}+\mathrm{l}_{4}\right)-D_{2}\left(\mathrm{l}_{1}+\mathrm{l}_{4}, \mathrm{l}_{2}+\mathrm{l}_{3}\right)\right]$

$\times\left[\mathcal{K}\left(\mathbf{l}_{1}, \mathbf{l}_{3} ; \mathbf{k}_{1}, \mathbf{k}_{3}\right) \delta\left(\mathbf{l}_{2}-\mathbf{k}_{2}\right) \delta\left(\mathbf{l}_{4}-\mathbf{k}_{4}\right)+\mathcal{K}\left(\mathbf{l}_{2}, \mathbf{l}_{4} ; \mathbf{k}_{2}, \mathbf{k}_{4}\right) \delta\left(\mathbf{l}_{1}-\mathbf{k}_{1}\right) \delta\left(\mathbf{l}_{3}-\mathbf{k}_{3}\right)\right]+$

${ }^{2}$ In that reference also the two-to-four kernel $K_{2 \rightarrow 4}$ was expressed in terms of the kernel $K_{2+2}$. The identity given there is not suited for deriving a new representation of the full vertex $V_{2 \rightarrow 4}$ in terms of full BFKL kernels as we give it here. We have found a different way to express $K_{2 \rightarrow 4}$ in terms of $K_{2 \rightarrow 2}$ which can be seen in the last two lines of equation (3.28) below. 
$+\left[D_{2}\left(\mathrm{l}_{1}+\mathrm{l}_{2}+\mathrm{l}_{4}, \mathrm{l}_{3}\right)+D_{2}\left(\mathrm{l}_{1}+\mathrm{l}_{3}+\mathrm{l}_{4}, \mathrm{l}_{2}\right)-D_{2}\left(\mathrm{l}_{1}+\mathrm{l}_{2}, \mathrm{l}_{3}+\mathrm{l}_{4}\right)\right.$

$\left.-D_{2}\left(\mathrm{l}_{1}+\mathrm{l}_{3}, \mathrm{l}_{2}+\mathrm{l}_{4}\right)\right]$

$\left.\times\left[\mathcal{K}\left(\mathbf{l}_{1}, \mathbf{l}_{4} ; \mathbf{k}_{1}, \mathbf{k}_{4}\right) \delta\left(\mathbf{l}_{2}-\mathbf{k}_{2}\right) \delta\left(\mathbf{l}_{3}-\mathbf{k}_{3}\right)+\mathcal{K}\left(\mathbf{l}_{2}, \mathbf{l}_{3} ; \mathbf{k}_{2}, \mathbf{k}_{3}\right) \delta\left(\mathbf{l}_{1}-\mathbf{k}_{1}\right) \delta\left(\mathbf{l}_{4}-\mathbf{k}_{4}\right)\right]\right\}$

$+\frac{g^{4}}{4(2 \pi)^{3}} \int\left(\prod_{i=1}^{3} d^{2} \mathrm{l}_{i}\right) \delta\left(\sum_{j=1}^{3} \mathrm{l}_{j}-\sum_{j=1}^{4} \mathrm{k}_{j}\right)$

$\times\left[D_{2}\left(l_{1}+l_{2}, l_{3}\right)-D_{2}\left(l_{1}+l_{3}, l_{2}\right)+D_{2}\left(l_{1}, l_{2}+l_{3}\right)\right]$

$\times\left\{\left[\mathcal{K}\left(\mathrm{l}_{1}, \mathrm{l}_{2} ; \mathrm{k}_{1}+\mathrm{k}_{2}, \mathrm{k}_{3}\right)-\mathcal{K}\left(\mathrm{l}_{\mathbf{l}}-\mathrm{k}_{1}, \mathrm{l}_{2} ; \mathrm{k}_{2}, \mathrm{k}_{3}\right)\right] \delta\left(\mathrm{l}_{3}-\mathrm{k}_{4}\right)\right.$

$-\left[\mathcal{K}\left(\mathbf{l}_{1}, \mathrm{l}_{3} ; \mathbf{k}_{1}+\mathrm{k}_{2}, \mathrm{k}_{4}\right)-\mathcal{K}\left(\mathbf{l}_{1}-\mathrm{k}_{1}, \mathrm{l}_{3} ; \mathbf{k}_{2}, \mathrm{k}_{4}\right)\right] \delta\left(\mathrm{l}_{2}-\mathrm{k}_{3}\right)$

$-\left[\mathcal{K}\left(\mathbf{l}_{1}, \mathbf{l}_{3} ; \mathbf{k}_{1}, \mathbf{k}_{3}+\mathrm{k}_{4}\right)-\mathcal{K}\left(\mathbf{l}_{1}, \mathbf{l}_{3}-\mathbf{k}_{4} ; \mathbf{k}_{1}, \mathbf{k}_{3}\right)\right] \delta\left(\mathbf{l}_{2}-\mathbf{k}_{2}\right)$

$\left.+\left[\mathcal{K}\left(\mathbf{l}_{2}, \mathbf{l}_{3} ; \mathbf{k}_{2}, \mathbf{k}_{3}+\mathrm{k}_{4}\right)-\mathcal{K}\left(\mathbf{l}_{2}, \mathbf{l}_{3}-\mathbf{k}_{4} ; \mathbf{k}_{2}, \mathbf{k}_{3}\right)\right] \delta\left(\mathbf{l}_{\mathbf{1}}-\mathbf{k}_{1}\right)\right\}$

$+\frac{g^{4}}{2(2 \pi)^{3}} \int\left(\prod_{i=1}^{2} d^{2} \mathrm{I}_{i}\right) \delta\left(\sum_{j=1}^{2} \mathrm{l}_{j}-\sum_{j=1}^{4} \mathrm{k}_{j}\right) D_{2}\left(\mathrm{l}_{1}, \mathrm{l}_{2}\right)$

$\times\left[\mathcal{K}\left(\mathrm{l}_{1}, \mathrm{l}_{2} ; \mathrm{k}_{1}+\mathrm{k}_{2}, \mathrm{k}_{3}+\mathrm{k}_{4}\right)-\mathcal{K}\left(\mathrm{l}_{1}-\mathrm{k}_{1}, \mathrm{l}_{2} ; \mathrm{k}_{2}, \mathrm{k}_{3}+\mathrm{k}_{4}\right)\right.$

$\left.-\mathcal{K}\left(\mathbf{l}_{1}, \mathrm{l}_{2}-\mathbf{k}_{4} ; \mathbf{k}_{1}+\mathrm{k}_{2}, \mathrm{k}_{3}\right)+\mathcal{K}\left(\mathrm{l}_{1}-\mathrm{k}_{1}, \mathrm{l}_{2}-\mathbf{k}_{4} ; \mathbf{k}_{2}, \mathrm{k}_{3}\right)\right]$

Since this formula is rather complicated we try to make it slightly more transparent by using a diagrammatic notation. We define a diagram for $\mathcal{K}$, the BFKL kernel including using a diagrammatic notation. We define a diagram
the propagators for the gluons entering from above,

$$
\mathcal{K}\left(\mathbf{q}_{1}, \mathbf{q}_{2} ; \mathbf{k}_{1}, \mathbf{k}_{2}\right)=\underbrace{\mathbf{q}_{1}}_{\mathbf{k}_{1}} \|_{\mathbf{k}_{2}}^{\mathbf{q}_{2}} .
$$

Let us further introduce a pictorial notation for the momentum arguments of the BFKL amplitude $D_{2}$. We write

$$
D_{2}\left(\mathbf{k}_{1}+\mathbf{k}_{2}, \mathbf{k}_{3}+\mathbf{k}_{4}\right)=D_{2}(\lambda \curlywedge),
$$

and the generalization of the notation to other combinations of the four momenta $\mathrm{k}_{i}$ is obvious. Now equation (3.28) can be rewritten as

$$
\begin{aligned}
& \left(V D_{2}\right)\left(\mathbf{k}_{1}, \mathbf{k}_{2} ; \mathbf{k}_{3}, \mathbf{k}_{4}\right)=
\end{aligned}
$$

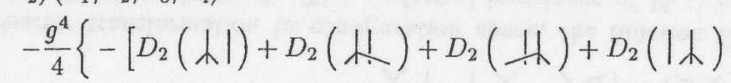

$$
\begin{aligned}
& \left.-D_{2}(\text { 人久 })-D_{2}(\text { ג })-D_{2}(\text { ג })\right] \times[(H \|)+(\| H)] \\
& +\left[D_{2}(\lambda \mid)+D_{2}(\mid \lambda)-D_{2}(\Lambda)\right] \times\left[\left(\left|\frac{1}{1}\right|\right)+\left(|| \frac{1}{1}\right)\right] \\
& +\left[D_{2}(\text { 岀 })+D_{2}(\text { 少 })-D_{2}(\text { 人久 })-D_{2}(\text { 从 })\right] \\
& \times[(\text { |III })+(\|H\|)]\} \\
& +\frac{g^{4}}{4}\left\{\left[D_{2}(\lambda \mid)-D_{2}(\text { 〈 })+D_{2}(\mid \lambda)\right] \times\right.
\end{aligned}
$$

$$
\begin{aligned}
& \times[(\text { HI) })-(\text { 凡I) })-(\text { 넾 })+(\text { hH })
\end{aligned}
$$

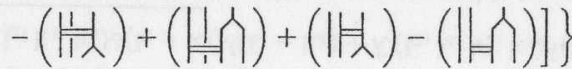

$$
\begin{aligned}
& \left.+\frac{g^{4}}{2} D_{2}(||) \times[(\text { 스 })-(\bigcap))-(h)+(h \wedge)\right] \text {. }
\end{aligned}
$$

Here an integration with the weight $1 /(2 \pi)^{3}$ over the loop momentum is implied as are the $\delta$-functions according to the gluon lines that are not involved in the interactions. 


\section{Chapter 4}

\section{Five Gluons}

In this chapter we do the next step in the investigation of unitarity corrections in high energy QCD. We want to study the five-gluon amplitude, thereby going now beyond previously known results. We prove that the five-gluon amplitude $D_{5}$ is the superposition of two-gluon amplitudes $D_{2}$ and irreducible four-gluon amplitudes $D_{4}^{I}$. This result constitutes a further generalization of the concept of reggeization and we will gain first insight on how reggeization works in higher $n$-gluon compound sto we first identify a reggeizing part $D_{5}^{R}$ in the full five-gluon amplitude and derive a new integral equation for the remaining part $D_{5}^{I}$. This step is technically very similar to the cor responding procedure for four gluons as we have outlined it in the preceding chapter Though, the computational effort necessary here is already considerable. In the case of four gluons we were able to iterate the integral equation for the remaining part and could read off the structure of the solution already at this stage. The situation in the five gluon amplitude is more complicated at this point. Fortunately, we will be able to write the in homogenes term of the new integral equation in terms of the known two-to-for solve the equation. Remarkably, the solution does not require an analytic expression fo the four-gluon compound state which we are still lacking. A very important piece in the solution of the integral equation for the remaining part $D_{5}^{I}$ is the mechanism leading to reggeization in a subsystem of three gluons. The reggeization in the three-gluon amplitude discussed in section 3.1 now turns out to be a special case of that more general mechanism of reggeization. We will be lead in a natural way to the conjecture that each am plitude with an odd number of $t$-channel gluons exhibits full reggeization. We conclude the chapter with a discussion of the results in the context of an effective field theory.

\subsection{A reggeizing part and the integral equation for the re-} maining part

In the first step, our analysis of the five-gluon amplitude $D_{5}$ follows very much the same lines as the study of the three- and four-gluon amplitudes. To get started we identify a reggeizing part $D_{5}^{R}$ of the amplitude and split the amplitude accordingly,

$$
D_{5}=D_{5}^{R}+D_{5}^{I} .
$$

With a well-chosen $D_{5}^{R}$ we will come to a new integral equation for the yet unknown quantity $D_{5}^{I}$. Again, this decomposition is not unique. Our ansatz will lead to an equation for $D_{5}^{I}$ that can even be solved. This situation is the best we can hope for and further justification for the ansatz is certainly not needed. The natural choice for $D_{5}^{R}$ is once more suggested by the inhomogeneous term $D_{(5 ; 0)}$. This means that our ansatz has exactly the same color and momentum structure as $D_{(5 ; 0)}$ in $(2.66)$, but we replace $D_{(2 ; 0)}$ by $D_{2}$ resulting in

$$
\begin{aligned}
& D_{5}^{R a_{1} a_{2} a_{3} a_{4} a_{5}}\left(\mathrm{k}_{1}, \mathrm{k}_{2}, \mathrm{k}_{3}, \mathrm{k}_{4}, \mathrm{k}_{5}\right)= \\
& =-g^{3}\left\{f^{a_{1} a_{2} a_{3} a_{4} a_{5}}\left[D_{2}(1234,5)+D_{2}(1,2345)-D_{2}(15,234)\right]\right. \\
& +\quad f^{a_{2} a_{1} a_{3} a_{4} a_{5}}\left[D_{2}(1345,2)-D_{2}(12,345)+D_{2}(125,34)-D_{2}(134,25)\right] \\
& +f^{a_{1} a_{2} a_{3} a_{5} a_{4}}\left[D_{2}(1235,4)-D_{2}(14,235)+D_{2}(145,23)-D_{2}(123,45)\right] \\
& +f^{a_{1} a_{2} a_{4} a_{5} a_{3}} \\
& {[}
\end{aligned}
$$

This is inserted into the integral equation (2.14). We insert into that equation the expressions $(3.1),(3.2),(3.3)$ for $D_{3}$ and $D_{4}$ as well. In order to find the new integral equation for $D_{5}^{I}$ we have to simplify and collect all terms not involving $D_{5}^{I}$ and $D_{4}^{I}$. These terms will contribute to the inhomogeneous term of the new equation for $D_{5}^{I}$ and we will now discuss them. From the left-hand side of (2.14) we get $\omega D_{5}^{R}$, which can be treated using the BFKL equation (2.11). Due to this, the inhomogeneous term $D_{(5: 0)}$ in $(2.14)$ is exactly cancelled and we get further terms involving only convolutions of $D_{2}$ functions with kernels $K_{2 \rightarrow 2}$ or trajectories $\beta$. From the right-hand side of $(2.14)$ we get contributions of the type $K_{2 \rightarrow 5} \otimes D_{2}, \sum K_{2 \rightarrow 4} \otimes D_{3}, \sum K_{2 \rightarrow 3} \otimes D_{4}^{R}$, and $\sum K_{2 \rightarrow 2} \otimes D_{5}^{R}$. All of these can be written as sums of convolutions of $D_{2}$ with the kernels $K_{2 \rightarrow m}$. The corresponding contractions of color tensors are performed using the diagrammatic method described in appendix A. In that appendix we also give the explicit formulae for some of the contractions required. The total number of contractions needed here is close to one hundred. Most of them can be obtained from those given in the appendix with a moderate amount of e momentum integrals are brought to their standard forms as cha 2.7. The respective momentum integrals and color contractions are then multiplied and can be collected. This last step amounts to collecting several thousand terms and sorting them according to the different color tensors. At least in this step the use of a computer algebra program is needed to cope with the large number of terms. In the analysis of the six-gluon amplitude in chapter 6 we will need computer power also in order to be able to deal with the huge number of momentum space integrals.

In the derivation of the new integral equation the terms involving $D_{4}^{I}$ remain unchanged. They will be treated at a later stage of the analysis. The same is true for the homogeneous term containing $D_{5}^{I}$. The combinations of the $D_{4}^{I}$ and $D_{5}^{I}$ amplitudes with the kernels are therefore the same as in the original equation (2.14). We thus find the following equation for the unknown part $D_{5}^{I}$ of the five-gluon amplitude:

$$
\left(\omega-\sum_{i=1}^{5} \beta\left(\mathrm{k}_{i}\right)\right) D_{5}^{I}=\sum f_{a_{1} a_{2} a_{3}} \delta_{a_{4} a_{5}} H(1,2,3 ; 4,5)+\sum K_{2 \rightarrow 3}^{\{b\} \rightarrow\{a\}} \otimes D_{4}^{I b_{1} b_{2} b_{3} b_{4}}
$$$$
+\sum K_{2 \rightarrow 2}^{\{b\} \rightarrow\{a\}} \otimes D_{5}^{I b_{1} b_{2} b_{3} b_{4} b_{5}} \text {. }
$$ 
The first term on the right hand side is the result of the computation described above. We will now treat it in more detail.

The first interesting observation concerns its color structure. All terms proportional to $f^{a_{1} a_{2} a_{3} a_{4} a_{5}}$ (and the other three permutations of this occurring in (4.2)) are cancelled between the different contributions to this inhomogeneous term and drop out. Something similar happened in the case of $D_{4}$ where there is no term proportional to $d^{a_{1} a_{2} a_{3} a_{4}}$ in the vertex $V_{2 \rightarrow 4}$ and only lower tensors (i. e. products of $\delta$-tensors) contribute.

Secondly, we observe the following symmetry of the new inhomogeneous term we have calculated. The sum extends over all (ten) possibilities to have a pair of gluons in a color singlet. For each of these permutations of the gluons color and momentum labels are exchanged simultaneously, i. e. the sum in (4.3) stands for

$$
\begin{aligned}
\sum f_{a_{1} a_{2} a_{3}} \delta_{a_{4} a_{5}} H(1,2,3 ; 4,5)= & f_{a_{1} a_{2} a_{3}} \delta_{a_{a_{a}} a_{5}} H(1,2,3 ; 4,5)+f_{a_{1} a_{2} a_{4}} \delta_{a_{3} a_{5}} H(1,2,4 ; 3,5) \\
& +\ldots+\delta_{a_{1} a_{2}} f_{a_{3} a_{4} a_{5}} H(3,4,5 ; 1,2) .
\end{aligned}
$$

The function $H$ is the same in all ten permutations. This symmetry is an outcome of our computation, and it has not been used to derive (4.3). On the other hand, it is not an unexpected property of the inhomogeneous term. Already in the corresponding equation (3.4) in the four-gluon case the inhomogeneous term, i.e. the vertex $V_{2 \rightarrow 4}$, had

A closer inspection of the function $H$ reveals that it is actually a superposition of vertex functions $V$ which we encountered in the discussion of the two-to-four vertex $V_{2 \rightarrow 4}$. Namely,

$$
H(1,2,3 ; 4,5)=\frac{g}{2}\left[\left(V D_{2}\right)(12,3 ; 4,5)-\left(V D_{2}\right)(13,2 ; 4,5)+\left(V D_{2}\right)(1,23 ; 4,5)\right] .
$$

To obtain this striking result is was necessary to go through the full calculation of all convolutions of amplitudes with kernels as described. It is only afterwards that we are able to discover the simple structure in terms of $V$. Unfortunately, we do not know a way leading to (4.3), (4.5) that avoids this tedious calculation.

\subsection{Solving the equation for the remaining part}

Up to this point, our analysis of the five-gluon amplitude followed essentially the same lines as in the case of four gluons. Whereas there the new integral equation could simply be iterated, this is not possible here. To find the solution for $D_{5}^{I}$ we now have to go beyond the procedure applied for $n=3$ and 4 gluons.

Taking a close look at the integral equation (4.3) for $D_{5}^{I}$ we discover that its structure bears a strong resemblance to the equation (2.12) for the three-gluon amplitude $D_{3}$. In the second term on the right hand side of (4.3) a pair of gluons of the amplitude $D_{4}^{I}$ is convoluted with two-to-three kernels. In the corresponding term in (2.12) it was the two-gluon (BFKL) amplitude $D_{2}$ that was convoluted with the same kernel. There the first term on the right hand side, i.e. $D_{(3.0)}$, was the superposition of quark loop amplitudes $D_{(2 ; 0)}$ that are the lowest order terms in the ladder expansion of $D_{2}$. In (4.3) the corresponding term (4.4) is, according to (4.5), the superposition of functions $V D_{2}$. (In fact it is even the superposition of full two-to-four reggeon vertices $V_{2 \rightarrow 4} D_{2}$ as we will see below.) These vertex functions, in turn, constitute the lowest order terms in the ladder expansion ${ }^{1}$ of the irreducible amplitude $D_{4}^{I}, \mathrm{cf}$. (3.13). More specifically, it is for each of the ten terms in (4.4) that we find in a three-gluon subsystem exactly the momentum structure that also determines $D_{(3 ; 0)}$. The similarity is also evident for the color structure, namely the three-gluon subsystem comes with a tensor $f_{a b c}$.

Clearly, this suggests to construct a solution $D_{5}^{I}$ in analogy to the three-gluon amplitude. While $D_{3}$ is a superposition of BFKL amplitudes $D_{2}$ we now should choose $D_{5}^{I}$ as a similar superposition of irreducible four-gluon amplitudes $D_{4}^{I}$. The following combination of $D_{4}^{I}$ amplitudes is of this kind and in fact is a solution to equation (4.3). We will outline the proof of this fact momentarily.

$$
\begin{aligned}
& D_{5}^{I a_{1} a_{2} a_{3} a_{4} a_{5}}\left(\mathrm{k}_{1}, \mathrm{k}_{2}, \mathrm{k}_{3}, \mathrm{k}_{4}, \mathrm{k}_{5}\right)=\frac{g}{2} \times \\
& \times\left\{f_{a_{1} a_{2} c} D_{4}^{I c a_{3} a_{4} a_{5}}(12,3,4,5)+f_{a_{1} a_{3} c} D_{4}^{I c a_{2} a_{4} a_{5}}(13,2,4,5)\right. \\
&+f_{a_{1} a_{4} c} D_{4}^{I c a_{2} a_{3} a_{5}}(14,2,3,5)+f_{a_{1} a_{5} c} D_{4}^{I c a_{2} a_{3} a_{4}}(15,2,3,4) \\
&+f_{a_{2} a_{3} c} D_{4}^{I a_{1} c a_{4} a_{5}}(1,23,4,5)+f_{a_{a_{4}} a_{4} c} D_{4}^{I I a_{1} c a_{3} a_{5}}(1,24,3,5) \\
&+f_{a_{2} a_{5} c} D_{4}^{I a_{1} c a_{3} a_{4}}(1,25,3,4)+f_{a_{3} a_{4} c} D_{4}^{I a_{1} a_{2} c a_{5}}(1,2,34,5) \\
&\left.+f_{a_{3} a_{5} c} D_{4}^{I a_{1} a_{2} c a_{4}}(1,2,35,4)+f_{a_{4} a_{5} c} D_{4}^{I a_{1} a_{2} a_{3} c}(1,2,3,45)\right\}
\end{aligned}
$$

In each of the terms one pair $(i, j)$ of gluons is merged ${ }^{2}$ into one gluon which then enters the irreducible four-gluon amplitude from below. This gluon in $D_{4}^{I}$ has momentum $\left(\mathrm{k}_{i}+\mathrm{k}_{j}\right)$ and color label $c$. The merging of the two gluons in color space happens via a $f_{a_{i} a_{j} c}$ tenso $(i<j)$. The position in the amplitude $D_{4}^{I}$ at which the 'composite' gluon with color $c$ and momentum $\left(\mathrm{k}_{i}+\mathrm{k}_{j}\right)$ enters does not matter since $D_{4}^{I}$ is completely symmetric in the four gluons, cf. (3.15). All possible pairs of gluons are treated in the same way. The way pairs of gluons are merged (or arise from splittings) becomes more transparent when (4.6) is written using birdtrack notation,

$$
\begin{aligned}
& D_{5}^{I a_{1} a_{2} a_{3} a_{4} a_{5}}\left(\mathbf{k}_{1}, \mathbf{k}_{2}, \mathbf{k}_{3}, \mathbf{k}_{4}, \mathbf{k}_{5}\right)=\frac{g}{2} \times \\
& \times\left\{[\lambda \mid \|] \star D_{4}^{I b_{1} b_{2} b_{3} b_{4}}(12,3,4,5)+[\lambda \mid \|] \star D_{4}^{I b_{1} b_{2} b_{3} b_{4}}(13,2,4,5)\right. \\
& +[\ldots|| \mid] \star D_{4}^{I b_{1} b_{2} b_{3} b_{4}}(14,2,3,5)+[\ldots||||] \star D_{4}^{I b_{1} b_{2} b_{3} b_{4}}(15,2,3,4) \\
& +[\|\lambda\|] \star D_{4}^{I b_{1} b_{2} b_{3} b_{4}}(1,23,4,5)+[|d| \mid] \star D_{4}^{I b_{1} b_{2} b_{3} b_{4}}(1,24,3,5) \\
& +[|\lambda|||] \star D_{4}^{I b_{1} b_{2} b_{3} b_{4}}(1,25,3,4)+[\| \lambda \mid] \star D_{4}^{I b_{1} b_{2} b_{3} b_{4}}(1,2,34,5) \\
& \left.+[\| \Lambda] \star D_{4}^{I b_{1} b_{2} b_{3} b_{4}}(1,2,35,4)+[\|\| \lambda] \star D_{4}^{I b_{1} b_{2} b_{3} b_{4}}(1,2,3,45)\right\} \text {. }
\end{aligned}
$$

${ }^{1}$ This statement has to be taken with some care, since the terms $V D_{2}$ are of course not of lowest orde in the coupling constant $g$. In the contrary, $D_{2}$ already contains an infinite series of ladder diagrams. What is meant here is that each diagram in the ladder expansion of $D_{4}^{Y}$ starts with a full two-gluon ladder and a vertex attached to this.

${ }^{2}$ Depending on the context one would like to use different words for the formula (4.6). From the poin of view of constructing the solution it is clearly a 'merging' of two gluons, with
evolution in mind one would prefer to speak of a 'splitting' of gluons. 
We will come to the interpretation of this structure later in this chapter in section 4.3.

Now we explain how (4.6) can be shown to solve the integral equation (4.3). The only pieces of information about the irreducible four-gluon amplitude $D_{4}^{I}$ we need for the purpose of this proof are its complete symmetry in the four outgoing gluons, cf. (3.15), and the integral equation (3.4) it fulfills. Fortunately, an analytic solution of the latter is not required.

We start from the integral equation (4.3) derived previously and insert the conjectured solution (4.6). On the left hand side then we find ten terms of the kind $\omega D_{4}^{I}$. To these we apply (3.4). Thereby we produce different terms and we first concentrate on the terms involving the vertex function $V$. For example, applying (3.4) to

$$
\omega f_{a_{1} a_{2} c} D_{4}^{I c a_{3} a_{1} a_{5}}(12,3,4,5)
$$

produces, due to (3.5), the expression

$$
\begin{aligned}
f_{a_{1} a_{2} c}\left(V_{2 \rightarrow 4} D_{2}\right)^{c a_{2} a_{3} a_{4}}(12,3,4,5)= & f_{a_{1} a_{2} a_{3}} \delta_{a_{4} a_{5}}\left(V D_{2}\right)(12,3 ; 4,5) \\
& +f_{a_{1} a_{2} a_{4}} \delta_{a_{3} a_{5}}\left(V D_{2}\right)(12,4 ; 3,5) \\
& +f_{a_{1} a_{2} a_{5}} \delta_{a_{3} a_{4}}\left(V D_{2}\right)(12,5 ; 3,4)
\end{aligned}
$$

containing three different vertex functions $V$. Similar expressions are obtained from the other $\omega D^{I}$ terms on the left hand side. In some cases a minus sign arises due to the antisymmetry of the structure constant $f_{a b c}$. For instance, from the second term in (4.6),

$$
f_{a_{1} a_{3} c} D_{4}^{I c a_{2} a_{4} a_{5}}(13,2,4,5)
$$

in which the pair $(1,3)$ of gluons is merged we get

$$
\begin{aligned}
& -f_{a_{1} a_{2} a_{3}} \delta_{a_{4} a_{5}}\left(V D_{2}\right)(13,2 ; 4,5)+f_{a_{1} a_{3} a_{4}} \delta_{a_{2} a_{5}}\left(V D_{2}\right)(13,4 ; 2,5) \\
& \quad+f_{a_{1} a_{3} a_{5}} \delta_{a_{2} a_{4}}\left(V D_{2}\right)(13,5 ; 2,4) .
\end{aligned}
$$

Therefore we find exactly the same thirty vertex functions that occur also on the right hand side of (4.3) according to (4.4), (4.5). We have thus confirmed that the conjectured solution (4.6) indeed reproduces the correct lowest order term in the integral equation, namely the combination of vertex functions $V D_{2}$ given above. Moreover, we see that the first term on the right hand side of the integral equation (4.3) is not only a superpositio of vertex functions $V D_{2}$ but of full transition vertices $V_{2 \rightarrow 4}$ (applied to $D_{2}$ as usual).

Let us now consider further terms in the integral equation (4.3) that we have not treated yet, namely those involving the irreducible four-gluon amplitude $D_{4}^{I}$. Having applied (3.4) to the $\omega D_{4}^{I}$ terms on the left hand side the homogeneous term of that equation produces convolutions of $D_{4}^{I}$ amplitudes with kernels $K_{2 \rightarrow 2}$. In these first a kernel acts on $D_{4}^{I}$ and then the splitting of one gluon into a pair happens according to the combinations in (4.6). On the right hand side of the integral equation (4.3) the last term also gives us convolutions of $D_{4}^{I}$ amplitudes with kernels $K_{2 \rightarrow 2}$, but here the order of the convolution and the splitting of gluons is interchanged: first one gluons splits into two and then two of the now five gluons interact via a kernel $K_{2 \rightarrow 2}$. Among the terms just mentioned a subclass cancels immediately. Consider the case that the two-to-two kernel acts between two gluons none of which undergoes a splitting (LHS) or has emerged from a splitting (RHS). Then the order of interaction and splitting along the $t$-channel evolution is irrelevant and these terms are in fact the same on both sides.
The next terms in the integral equation that we look at are the products of $D_{4}^{I}$ 's with trajectories $\beta$. These arise on the left hand side either from $\omega D_{4}^{I}$ via (3.4) or from the original $\left[\sum_{i=i}^{5} \beta\left(\mathbf{k}_{i}\right)\right] D_{5}^{I}$ after (4.6) is inserted. Those in which the argument of the trajectory function does not correspond to a gluon undergoing or arising from a splitting cancel directly between these contributions. It can be easily checked that the others are exactly cancelled by the terms from the right hand side in which the two gluons emerging from a splitting interact with each other via a kernel $K_{2 \rightarrow 2}$.

It is a bit more complicated to study the expressions still left in the integral equation after the cancellations discussed so far. These are $D_{4}^{I}$ 's undergoing a two-to-three transition via the kernel $K_{2 \rightarrow 3}$ on the RHS and convolutions of $D_{4}^{I}$ functions with kernels $K_{2 \rightarrow 2}$ in which one of the gluons undergoing or emerging from a splitting is involved in $K_{2 \rightarrow 2}$ in which one of the gluons undergoing or emerging fom a sponvelutions in which the interaction (both sides). The latter do no longer include such convolutions in which der consideration three of the five outgoing gluons participate in the splitting or in the interaction. The other two gluons do not interact and can be in an arbitrary color state. Among the five gluons there can be a total of ten different three-gluon subgroups, and we will argue that the cancellation takes place in each of these subgroups separately. To this end let us concentrate on one of these subgroups, say the one with the first three of the outgoing gluons affected.

The mechanism that makes these contributions cancel between the two sides of the integral equation is the same that already caused reggeization in the three-gluon amplitude $D_{3}$. This does not come as a surprise since it was just the similarity of the corresponding integral equations that lead us to the ansatz (4.6). The identity actually bringing about the reggeization of the three-gluon subsystem is in pictorial language

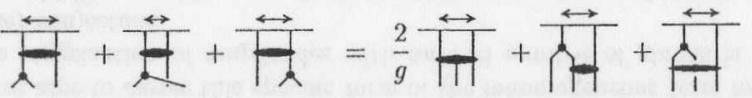

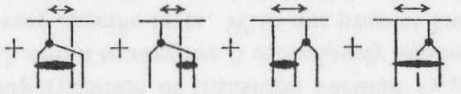

Only the three-gluon subsystem is shown, and the horizontal lines at the top are mean to suggest the irreducible amplitude $D_{4}^{I}$ that the gluons enter. The arrows indicate the symmetry of this amplitude under the simultaneous exchange of color and momentum of the two gluons. The kernels are the ones defined in section 2.6 .

The splitting of a gluon is depicted here by the corresponding color diagram and is meant to indicate the behavior in momentum space ${ }^{3}$ as well. The terms on the left (right) hand side of (4.12) are exactly the ones that occur on the left (right) hand side of the integral equation (4.3). To prove (4.12), the convolutions are evaluated as described i the preceding sections. However, here the situation is slightly complicated by the fact that the two gluons entering from above can be in an arbitrary color state. In the case of $D_{3}$ these two gluons were in a color singlet state, effectively reducing all color tensors to an overall $f_{a} a_{3}$. Here we have to be more careful and treat three independent color classes separately. (Of course, this could have been done already for $D_{3}$ but there it was

${ }^{3}$ Strictly speaking, this is done in abuse of our notation that usually separates momentum and color space. Confusion should hardly be possible here as all terms have been described in detail before. 
not necessary.) The three classes are

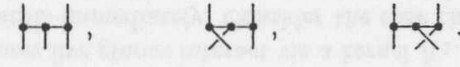

For some of the terms in (4.12) it is necessary to use the symmetry in the upper two gluons which is a property of $D_{4}^{I}$. Each of the three terms on the left hand side of (4.12) contributes to two of the three color classes in (4.13) via the Jacobi identity (2.55). Having dissected the integral equation this far, it is finally a comparatively short calculation to check that (4.12) holds. Thereby we have finished the proof that $D_{5}^{I}$ as given in (4.6) in fact is the solution of the integral equation.

\subsection{Interpretation of the result}

In this chapter we have been quite successful in dealing with the integral equation for the five-gluon amplitude. It was even possible to solve the equation. Now we want to interpret our findings in view of a possible field theory of unitarity corrections. Let us first summarize the essential results we have obtained in this chapter. We have split the five-gluon amplitude into two parts. The first part was the reggeizing part $D_{5}^{R}$ that is the superposition of two-gluon amplitudes $D_{2}$. We have found an integral equation is the superposition of two-gluon amplitudes $D_{2}$. We have found an integral equation
for the remaining part $D_{5}^{I}$ and have solved it. It turned out that the remaining part is the superposition of irreducible four-gluon amplitudes $D_{4}^{I}$. Neglecting all normalization factors and color tensors, this situation can be sketched in the following way:

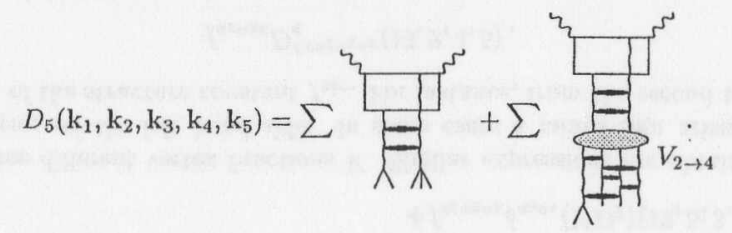

The first term on the right hand side is the reggeizing part $D_{5}^{R}$ of the amplitude (see (4.2)). The sum extends over all partitions of the five gluons into two groups. The second term is the one found in the preceding section. Here the sum includes all possible pairs of gluons that then merge into one.

On first sight, it may seem that we did not discover anything new here. We only find the known amplitudes $D_{2}$ and $D_{4}^{I}$, and the well-known two-to-four transition vertex $V_{2 \rightarrow 4}$ There is no new transition vertex and no new irreducible amplitude that would include a five-gluon compound state. But exactly this 'nothing' is quite an intriguing result. constitutes a gener constitutes a generalization of the concept of reggeization and proves that reggeization also takes place in more complicated amplitudes. Especially interesting is the reggeization in the second part that turns out to be a superposition of irreducible four-gluon amplitudes. In this part all possible pairs of gluons can merge into one more composite reggeon and we have to sum over all these pairs. The mechanism at work here is therefore exactly the same as in the three-gluon amplitude.

Reggeization was an important prerequisite for the emergence of the two-to-four vertex and thus of the field theory structure in the four-gluon amplitude. That the phenomenon of reggeization also occurs in the five-gluon amplitude gives us some confidence that the idea of a field theory structure will be a good guiding line also for the investigation of the six-gluon amplitude.

Given the fact that the three- and five-gluon amplitudes exhibit complete reggeization caused by the same mechanism, one is naturally lead to the question whether the same is true for each odd number $n$ of gluons. Indeed, the mechanism leading to reggeization in a three-gluon subsystem is very general. It is completely independent of the structure of the quark loop that we started with in the analysis of the integral equations. In deriving (4.12) we only made use of the fact that the amplitude to which the upper two gluons are attached - in this case $D_{4}^{I}$ - is symmetric in the two gluons. Therefore we can conclude that for a given odd $n$ one important condition for the reggeization of the $n$ gluon amplitude is fulfilled as soon as the irreducible part of the $(n-1)$-gluon amplitude is symmetric. However, we have to keep in mind that this is only one of the two conditions leading to complete reggeization. The second condition necessary for the reggeization of $D_{5}^{I}$ was that the inhomogeneous term in the integral equation (4.3) had the specific form (4.5), i. e. could be written as a special superposition of transition vertices $V_{2 \rightarrow 4}$. For a part of an arbitrary $n$-gluon amplitude with odd $n$ to reggeize it is obviously necessary that the respective inhomogeneous term has a very specific form. With our present knowledge, we are not able to derive this specific form of the inhomogeneous term for general $n$. The complete reggeization of amplitudes with an od (plausible) conjecture.

We would like to add a remark that concerns our choice of notation. Actually, this remark goes beyond a pure issue of notation. In the splitting of the four-gluon amplitude into two parts in (3.2) the superscript $I$ in $D_{4}^{I}$ was meant to indicate that this part of the amplitude is irreducible - in contrast to the other part. For four gluons this was a good choice of notation since that part in fact contains a new irreducible compound state of four reggeized gluons. In the case of five gluons, we again split the amplitude into two parts, cf. (4.1). The first part $D_{5}^{R}$ is a reggeizing one as the superscript $R$ indicates. But now we have discovered that the remaining patt $D^{I}$ re now we heve when extended in an appropriate way. The first supc as specifying the (non-)reggeization of the respective part with respect to the two-gluon state. We can then introduce a second superscript to indicate the (non-)reggeization with respect to the four-gluon state. (We will actually be forced to do so when considering the six-gluon amplitude in chapter 6.) For a proper notation we should thus identify

$$
D_{5}^{I}=D_{5}^{I, R}
$$

the second superscript now telling us that this part reggeizes with respect to the four-gluon state. The notation can easily be extended to accommodate reggeization with respect to a potential six-gluon state or even higher compound states. 


\section{Chapter 5}

\section{Ward Type Identities and Reggeization Tensors}

Equipped with solutions of the integral equations for up to five $t$-channel gluons we are now in a position to study two novel aspects of their structure. Both concern the intricat interplay of color and momentum structure present in the amplitudes. This interplay in the high energy limit reflects the non-abelian character of QCD. It is thus expected to reside at the very center of the field theory structure we are heading for. As we have seen, the phenomenon of reggeization is intimately related to the emergence of a field theory structure and actully a prerequite for it. With the results of the prest chapter, we structure and actinly a pre deeper for it. hope to do a ste towards a deeper understanding of this relation. It is therefore quite natural that both aspects we want to investigate here deal with reggeization. The first aspect is the subject of section 5.1. It is of a more global nature and relates $n$-gluon amplitudes of different $n$. In section 5.2 we encounter more local properties, namely the color tensors accompanying the reggeization of a single gluon.

So far we only know the structure of the amplitudes with up to five $t$-channel gluons. Our aim in the present chapter is to extract from this limited set of results as much information as possible. We then try to formulate conjectures how the observations made infore here can be generalized not be able to decide which of the results found here are theory and which depend on the special process under consideration, i.e. on the coupling of the $n$ gluons to the photon via a quark loop. It is also conceivable that some of the results are only valid for amplitudes with a small number of gluons and in general have to be replaced by more involved relations. To settle these questions requires either to derive similar results from a different starting point, for instance within an effective action approach $[39,40]$, or the investigation of amplitudes with more gluons in the $t$-channel. In this thesis, the study of the six-gluon amplitude in the next chapter will offer the first opportunity to put our conjectures to the test.

\subsection{Ward type identities}

The Ward type identities to be discussed in this section relate $n$-gluon amplitudes of different $n$ and allow to gain further insight into the interplay between their color and momentum structure. These identities of Ward type arise when we set one of the $n$ gluon momenta $\mathrm{k}_{i}$ to zero. Roughly speaking, the amplitude $D_{n}$ can in this case be expressed in terms of the amplitude $D_{n-1}$. The reduction is accompanied by an interesting behavior of (1) cor which we can extract a ge in color space does not only involve the gluons to which the one with vanishing momentum is coupled in the original amplitude (for example in the reggeizing parts) but involves all gluons of the amplitude. In this sense our identities constitute a global property of the amplitudes.

We will be able to find a formula valid for the reggeizing parts $D_{n}^{R}$ of the amplitudes and for these we can even give a general proof for arbitrary $n$. For the remaining parts $D_{n}^{\prime}$ we limit our study to the cases $n=4,5$ as we know them from the preceding chapters. We will then make a conjecture on how the the mechanisn works for higher $n$ here. If it the field theory structure of unitarity corrections. Specifically, we will find a characteristic difference between the parts of the amplitudes that exhibit reggeization and such parts that do not. Moreover, the identities obviously lead to strong constraints on the amplitudes. Both facts might turn out to be very helpful especially for the investigation of higher $n$-gluon amplitudes with $n \geq 6$ where a more complicated structure is expected to arise. We will discuss the potential significance of the Ward type identities for the field theory structure in more detail in subsection 5.1.3.

We start with considering the reggeizing parts $D_{n}^{R}$ and study for each $n$ how the color tensors rearrange in the case of a vanishing momentum $k_{i}$. It seems to us quite instructive to see the mechanism at work in concrete examples. With these we also hope to convey the impression that the Ward type identities impose very strong constraints on the color and momentum structure of the amplitudes. After that we state the general rule for the amplitudes $D_{n}^{R}$ in (5.17), (5.18) and sketch the proof for arbitrary $n$. Then we turn to the amplitudes $D_{4}^{I}$ and $D_{5}^{I}$ and formulate the conjecture how higher $D_{n}^{I}$ have to be treated.

\subsubsection{The reggeizing parts $D_{n}^{R}$}

After recalling that the BFKL amplitude $D_{2}$ vanishes in case one of its momentum arguments vanishes (see $(2.8)$ )

$$
\left.D_{2}\left(\mathrm{k}_{1}, \mathrm{k}_{2}\right)\right|_{\mathbf{k}_{1}=0}=\left.D_{2}\left(\mathrm{k}_{1}, \mathrm{k}_{2}\right)\right|_{\mathbf{k}_{2}=0}=0,
$$

we consider first the reggeizing parts $D_{n}^{R}$ of the amplitudes $D_{n}$, with $n$ ranging from 3 to 5. This includes also the full amplitude $D_{3}$ since it consists of reggeizing pieces only. The simplest relations hold for the case in which we set the first momentum $\mathbf{k}_{1}=0$, namely the vanishing of the amplitudes ${ }^{1}$

$$
\left.D_{3}\right|_{\mathbf{k}_{1}=0}=\left.D_{4}^{R}\right|_{\mathbf{k}_{1}=0}=\left.D_{5}^{R}\right|_{\mathbf{k}_{1}=0}=0 .
$$

The same is true for setting the $n$th (i.e. the last) momentum to zero in the amplitude $D_{n}^{R}$,

$$
\left.D_{3}\right|_{\mathrm{k}_{3}=0}=\left.D_{4}^{R}\right|_{\mathbf{k}_{4}=0}=\left.D_{5}^{R}\right|_{\mathbf{k}_{5}=0}=0 .
$$

'Stiction since the amplitudes $D_{n}$ are for different $n$ objects in different $Q_{[}\left[s u\left(N_{c}\right)\right]$ tensor spaces. 
We will see below that the identities (5.2) and (5.3), although seemingly trivial, fit well into the more general rule that determines the color structure of our Ward-type identities. When setting one of the momenta $k_{2}, \ldots, k_{n-1}$ to zero the amplitudes do not vanish. For the three gluon amplitude we fin

$$
\begin{aligned}
\left.D_{3}^{a_{1} a_{2} a_{3}}\right|_{\mathbf{k}_{2}=0} & =g f_{a_{1} a_{2} a_{3}} D_{2}\left(\mathbf{k}_{1}, \mathbf{k}_{3}\right) \\
& =g\left[\lfloor\mid] \star D_{2}^{b_{1} b_{2}}\left(\mathbf{k}_{1}, \mathbf{k}_{3}\right)=g[|\downarrow|] \star D_{2}^{b_{1} b_{2}}\left(\mathbf{k}_{1}, \mathbf{k}_{3}\right)\right.
\end{aligned}
$$

In the second line we have used the 2-gluon amplitude including color labels, that is $D_{2}^{b_{1} b_{2}}=\delta_{b_{1} b_{2}} D_{2}$ (see (2.7)). The way the color structure is written in the second line serves to make the general rule for the color structure more transparent. For the reggeizing part $D_{4}^{R}$ of the four-gluon amplitude we find

$$
\begin{aligned}
\left.D_{4}^{R a_{1} a_{2} a_{3} a_{4}}\right|_{\mathbf{k}_{2}=0} & =g f_{a_{1} a_{2} c} D_{3}^{c a_{3} a_{4}}\left(\mathbf{k}_{1}, \mathrm{k}_{3}, \mathrm{k}_{4}\right) \\
& =g[\lambda||] * D_{3}^{b_{3} b_{2} b_{3}}\left(\mathbf{k}_{1}, \mathbf{k}_{3}, \mathbf{k}_{4}\right) .
\end{aligned}
$$

This can be seen directly from the definition of $D_{4}^{R}$. For $\mathrm{k}_{2}=0$ the two expressions in square brackets in (3.3) become equal due to (2.8) and the color tensor in (5.6) is the difference of the two color tensors in (3.3),

$$
d^{a b c d}-d^{b a c d}=-\frac{1}{2} f_{a b k} f_{k c d},
$$

as follows from (2.26). The color tensor corresponding to $D_{3}$, i.e. $f_{b_{1} b_{2} b_{3}}$, is an invarian tensor. According to (2.54) the second line in (5.6) can thus also be written as

$$
\left.D_{4}^{R a_{1} a_{2} a_{3} a_{4}}\right|_{\mathbf{k}_{2}=0}=g\left[|\lambda|+|||| \ldots D_{3}^{b_{1} b_{2} b_{3}}\left(\mathbf{k}_{1}, \mathbf{k}_{3}, \mathbf{k}_{4}\right) .\right.
$$

For $\mathbf{k}_{3}=0$ we find in the same way

$$
\begin{aligned}
\left.D_{4}^{R a_{1} a_{2} a_{3} a_{4}}\right|_{\mathbf{k}_{3}=0} & =g f_{a_{3} a_{4} c} D_{3}^{a_{1} a_{2} c}\left(\mathrm{k}_{1}, \mathrm{k}_{2}, \mathrm{k}_{4}\right) \\
& =g\left[\||\lambda| \star D_{3}^{b_{1} b_{2} b_{3}}\left(\mathbf{k}_{1}, \mathbf{k}_{2}, \mathrm{k}_{4}\right)\right. \\
& =g[|\lambda|+|| \mid] \star D_{3}^{b_{1} b_{2} b_{3}}\left(\mathrm{k}_{1}, \mathrm{k}_{2}, \mathrm{k}_{4}\right) .
\end{aligned}
$$

The Ward identities for the reggeizing part $D_{5}^{R}$ of the five-gluon amplitude arise in a similar way. Setting one of the outgoing momenta to zero in (4.2), we find that the four different momentum structures reduce to two. When $k_{2}=0$, for instance, the expressions in square brackets in line 1 and 2 in (4.2) become equal up to a sign, as do the expressions in square brackets in line 3 and 4 . The corresponding pairs of color tensors can be added using (2.22),

$$
\begin{aligned}
& f^{a_{1} a_{2} a_{3} a_{4} a_{5}}-f^{a_{2} a_{1} a_{3} a_{4} a_{5}}=f_{a_{1} a_{2} c} d^{c a_{3} a_{4} a_{5}} \\
& f^{a_{1} a_{2} a_{3} a_{5} a_{4}}-f^{a_{1} a_{2} a_{4} a_{5} a_{3}}=f_{a_{1} a_{2} c} d^{a_{3} c a_{4} a_{5}}
\end{aligned}
$$

Comparing with (3.3) we can thus write

$$
\begin{aligned}
& \left.D_{5}^{R a_{1} a_{2} a_{3} a_{4} a_{5}}\right|_{\mathbf{k}_{2}=0}=g f_{a_{1} a_{2} c} D_{4}^{R c a_{3} a_{4} a_{5}}\left(\mathbf{k}_{1}, \mathbf{k}_{3}, \mathbf{k}_{4}, \mathbf{k}_{5}\right) \\
& =g[\lambda|| l] \star D_{4}^{R b_{1} b_{2} b_{3} b_{4}}\left(\mathbf{k}_{1}, \mathbf{k}_{3}, \mathbf{k}_{4}, \mathbf{k}_{5}\right) \\
& =g\left[||||+||||+|||||\ldots| D_{4}^{R b_{1} b_{2} b_{3} b_{4}}\left(\mathbf{k}_{1}, \mathbf{k}_{3}, \mathbf{k}_{4}, \mathbf{k}_{5}\right) \cdot(5.12)\right. \\
& \left.D_{5}^{R a_{1} a_{2} a_{3} a_{4} a_{5}}\right|_{\mathbf{k}_{4}=0}=g f_{a_{4} a_{5} c} D_{4}^{R a_{1} a_{2} a_{3} c}\left(\mathbf{k}_{1}, \mathbf{k}_{2}, \mathbf{k}_{3}, \mathbf{k}_{5}\right) \\
& =g[\|\| \downarrow] \star D_{4}^{R b_{1} b_{2} b_{3} b_{4}}\left(\mathbf{k}_{1}, \mathbf{k}_{2}, \mathbf{k}_{3}, \mathbf{k}_{5}\right) \\
& =g\left[\|||+\mid\lfloor|+||| \mid] \star D_{4}^{R b_{1} b_{2} b_{3} b_{4}}\left(\mathrm{k}_{1}, \mathrm{k}_{2}, \mathrm{k}_{3}, \mathrm{k}_{5}\right) \cdot(5.13)\right.
\end{aligned}
$$

For $\mathrm{k}_{4}=0$ similarly

The last lines in (5.12) and (5.13) are again implied by the fact that the color tensors in $D_{4}^{R b_{1} b_{2} b_{3} b_{4}}$ are invariant tensors. For $\mathrm{k}_{3}=0$ we need the analogue of $(5.10),(5.11)$ which is slightly more complicated. Applying (2.22) two times we get the two identities

$$
\begin{aligned}
& f^{a_{1} a_{2} a_{3} a_{4} a_{5}}-f^{a_{1} a_{2} a_{4} a_{5} a_{3}}=f_{a_{1} a_{3} c} d^{c a_{2} a_{4} a_{5}}+f_{a_{2} a_{3} c} d^{a_{1} c a_{4} a_{5}} \\
& f^{a_{2} a_{1} a_{3} a_{4} a_{5}}+f^{a_{1} a_{2} a_{3} a_{5} a_{4}}=f_{a_{1} a_{3} c} d^{a_{2} c a_{4} a_{5}}+f_{a_{2} a_{3} c} d^{c a_{1} a_{4} a_{5}} .
\end{aligned}
$$

Using this we get from the formula (4.2) for the reggeizing part

$$
\begin{aligned}
\left.D_{5}^{R a_{1} a_{2} a_{3} a_{4} a_{5}}\right|_{\mathbf{k}_{3}=0} & =g f_{a_{1} a_{3} c} D_{4}^{R c a_{2} a_{4} a_{5}}\left(\mathbf{k}_{1}, \mathbf{k}_{2}, \mathbf{k}_{4}, \mathbf{k}_{5}\right)+g f_{a_{2} a_{3} c} D_{4}^{R a_{1} c a_{4} a_{5}}\left(\mathbf{k}_{1}, \mathbf{k}_{2}, \mathbf{k}_{4}, \mathbf{k}_{5}\right) \\
& =g[\lambda|||| \lambda \|] \star D_{4}^{R b_{1} b_{2} b_{3} b_{4}}\left(\mathbf{k}_{1}, \mathbf{k}_{2}, \mathbf{k}_{4}, \mathbf{k}_{5}\right) \\
& =g[\|\lambda \mid+\| \|] \star D_{4}^{R b_{1} b_{2} b_{3} b_{4}}\left(\mathbf{k}_{1}, \mathbf{k}_{2}, \mathbf{k}_{4}, \mathbf{k}_{5}\right) .
\end{aligned}
$$

The Ward identities collected here for the reggeizing parts $D_{n}^{R}$ of the $n$-gluon amplitudes $(n \geq 3)$ can be summarized as follows. For vanishing momentum $\mathrm{k}_{i}$ the momentum part of the amplitude $D_{n}^{R}$ reduces to $D_{n-1}^{R}$, the momentum arguments being the $(n-1)$ remaining transverse momenta. (Here we again identify $D_{3}^{R}=D_{3}$ since $D_{3}$ reggeizes completely, and $D_{3-1}^{R}$ should be understood as $D_{2}$.) In color space the label $a_{i}$ of the

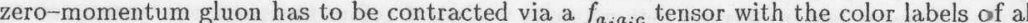
作 $j$ to the left, that is with $j<i$ and $c$ has to be taken at the $j$ th position in the amplitude $D_{n-1}$. Since the amplitudes $D_{n-}^{R}$ all color labels $a_{j}$ to the right $(j>i)$ with a $f_{a_{i} a_{j} c}$ tensor. In the latter case the label $c$ is at the $(j-1)$ th position in the amplitude $D_{n-1}^{R}$. Casting this into formulae we have

$$
\begin{aligned}
& \left.D_{n}^{R a_{1} \ldots a_{n}}\left(\mathrm{k}_{1}, \ldots, \mathrm{k}_{n}\right)\right|_{\mathrm{k}_{i}=0}= \\
& =g \sum_{j=1}^{i-1} f_{a_{j} a_{i} c} D_{n-1}^{R a_{1} \ldots \hat{a}_{j} c_{j} \ldots \hat{a}_{i} \ldots a_{n}}\left(\mathbf{k}_{1}, \ldots, \widehat{\mathbf{k}}_{i}, \ldots, \mathbf{k}_{n}\right) \\
& =g \sum_{j=i+1}^{n} f_{a_{i} a_{j} c} D_{n-1}^{R a_{1} \ldots \hat{a}_{i} \ldots \hat{a}_{j} \ldots a_{n}} \underset{\hat{\dagger}_{j-1}}{\mathrm{k}_{j}}\left(\mathrm{k}_{1}, \ldots, \hat{\mathrm{k}}_{i}, \ldots, \mathrm{k}_{n}\right) \text {, }
\end{aligned}
$$


where the hat indicates that the corresponding quantity has to be left out. The formulae include the special cases $\mathbf{k}_{1}=0$ and $\mathbf{k}_{n}=0$ as well: the respective sum in (5.17) or $(5.18)$ is empty or it contains $(n-1)$ terms and vanishes due to the condition for invarat (5.18) ensen (2.54). The Ward amplitude can even be shown to hold for arbitrary $n$ (see below). This is of course only true in the case that we choose the reggeizing part $D_{n}^{R}$ to be obtained from the corresponding quark loop $D_{(n ; 0)}$ by replacing $D_{(2 ; 0)} \rightarrow D_{2}$ as discussed in section 3. Other choices for $D_{n}^{R}$ in the decomposition into reggeizing part and irreducible part of the $n$-gluon amplitude will in general violate the Ward identities.

In the preceding chapters explicit formulae for the reggeizing amplitudes have been displayed only for up to five gluons. In addition, we will encounter the reggeizing part $D_{6}^{R}$ of the six-gluon amplitude in the next chapter (see (6.2)). It can also be shown to fulfill the Ward type identities (5.17), (5.18) following the same lines as for up to five gluons. We even have the possibility to prove the Ward type identities for arbitrary $n$. Due to the construction of the amplitudes $D_{n}^{R}$ from the quark loop (by the replacement $D_{(2 ; 0)} \rightarrow D_{2}$ ) it is sufficient to prove the identities for $D_{(n ; 0)}$. The quark loop is the sum of $2^{n}$ diagrams ${ }^{2}$ as explained in section 2.4. Consider now two of these diagrams that differ in the coupling of the $i$ th gluon. It is coupled to the quark line in one and to the antiquark line in the other diagram. Due to this the two diagrams have opposite sign. But otherwise the momentum

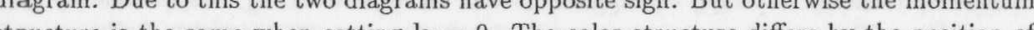
structre is the the generato $t^{a_{i}}$ around the loop to the left (or to the right) by iterated use of (2.22). Doing so we come across all gluons $j$ with $j<i$ since the cuts in the amplitude (see fig. 2.1) forbid crossing of $t$-channel gluons. Therefore this procedure generates exactly the terms needed for (5.17) containing a trace over $(n-1)$ generators contracted with a $f_{a j a_{i} c}$. (Although it is a bit tedious, the correct signs can be checked without difficulty.) The two remaining terms with a trace over $n$ generators cancel due to the sign mentioned above. The second form of the Ward identity (5.18) is obtained by shifting the generator $t^{a_{i}}$ around the loop to the right instead of to the left.

\subsubsection{The amplitudes $D_{4}^{I}$ and $D_{5}^{I}$}

We now come to examining the Ward identities for that part of the $n$-gluon amplitude which is not the superposition of two-gluon amplitudes. This requires $n \geq 4$ since only in this case we have a non-vanishing part $D_{n}^{I}$ as defined in the preceding chapters. On the other hand our knowledge of this part is rather limited: it is only up to $n=5$ that we know its structure, and even here we do not have an analytic formula for the four-gluon state. Therefore we have to restrict ourselves to $D_{4}^{I}$ and $D_{5}^{I}$ here.

For $D_{4}^{I}$ we have already found (see (3.14)) that it vanishes whenever one of the momentum argument vanishes. The study of the amplitude $D_{5}^{I}$ is surprisingly simple. fulfills Ward identities very similar to those valid for the reggeizing amplitudes $D^{R}$. I expect. Whereas the amplitude $D_{n}^{R}$ was reduced to a superposition of $D_{n-1}^{R}$ 's when one momentum was set to zero, $D_{5}^{I}$ now reduces to a sum of $D_{4}^{I}$ amplitudes. In detail, we find

$$
\begin{aligned}
& \left.D_{5}^{I a_{1} \ldots a_{5}}\left(\mathbf{k}_{1}, \ldots, \mathrm{k}_{5}\right)\right|_{\mathbf{k}_{i}=0}= \\
& \quad=g \sum_{j=1}^{i-1} f_{a_{j} a_{i} c} D_{4}^{I a_{1} \ldots \hat{a}_{j} \ldots \ldots \hat{a}_{1} \ldots a_{5}}\left(\mathbf{k}_{1}, \ldots, \widehat{\mathbf{k}}_{i}, \ldots, \mathbf{k}_{5}\right) \\
& \quad=g \sum_{j=i+1}^{5} f_{a_{i} a_{j} c} D_{4}^{I a_{1} \ldots \hat{a}_{i} \ldots c \hat{a}_{j} \ldots a_{n}}\left(\mathrm{k}_{1}, \ldots, \widehat{\mathrm{k}}_{i}, \ldots, \mathbf{k}_{5}\right)
\end{aligned}
$$

for any $i \in\{1, \ldots, 5\}$. Once we have found the amplitude $D_{5}^{I}$ in the form (4.6), only two more ingredients are required for the proof. One is the vanishing of $D_{4}^{I}$ for vanishing argument (3.14), the other is the defining property (2.54) of invariant su $\left(N_{c}\right)$ tensors. The latter applies here since the inglet. With these two pieces of information at hand the calculation leading to (5.19) and $(5.20)$ is almost trivial.

\subsubsection{Significance of the Ward type identities}

The Ward identities discussed so far suggest an underlying pattern also valid for higher $n$-gluon amplitudes. In this section we try to make an educated guess about the generalization of the identities to higher $n$. This is done in the form of several conjectures. Each of the conjectures applies to a certain part of the amplitudes, for instance to the part of a $n$-gluon amplitude that reggeizes into two-gluon amplitudes. Since the integral equations only constrain the full amplitudes $D_{n}$, we will not be able to prove the conjectures for the different parts separately. The main conjecture we make here is actually that it is possible at all to split the amplitudes into different parts in such a way that the latter fulfill the conjectures below. This main conjecture, however, can very well be put at tes by investigating amplitudes with more $t$-channel gluons or by deriving the field theory structure from a different starting point. With this situation in mind, we now state what we expect for the different parts of the amplitudes.

Our first conjecture is that the number-changing vertices of the effective field theory (as they result from the appropriate splitting of the amplitudes into reggeizing and non-reggeizing parts) vanish when one of the outgoing gluons has vanishing transvers momentum. It is certainly true (see (3.10)) for the two-to-four transition vertex $V_{2 \rightarrow}$ which is up to now the only known number-changing element. In order to find out abou other number-changing elements we have to study higher $n$-gluon amplitudes. This wil be done in the next chapter for the six-gluon amplitude where we will try to find out about a possible two-to-six transition. Unfortunately, we will not be able to arrive at a final clarification of this issue. This is due to other conceptional problems we will come across in that case. Here we mention only that these problems are connected with finding a proper definition of what can be called an 'element' of the field theory and refer the reader to section 6.3 .2 for more detailed discussion.

Connected with the preceding one is the conjecture that the non-reggeizing parts of the amplitudes vanish if one of the outgoing gluons has zero momentum. Examples found so far are the two-gluon (BFKL) amplitude $D_{2}$ and the irreducible part $D_{4}^{I}$ of the four-gluon amplitude. 
The final and probably the most important conjecture concerns the reggeizing parts of the amplitudes. If some part of a $n$-gluon amplitude can be written as a superposition of non-reggeizing parts of lower amplitudes it should satisfy the Ward identities found in the previous sections. However, we know from the way we have treated the amplitude so far that the choice of a reggeizing part is at our disposal. Therefore, this conjecture amounts to a condition for a good choice of a reggeizing part. This brings us back to the problem already mentioned in section 3.2 and we will now discuss it in more detail.

There is some freedom in decomposing the amplitudes $D_{n}$ into a reggeizing and remaining part as discussed in chapter 3 . Our conjectures about the Ward identities should be used as conditions for the sensible decomposition of the amplitudes into reggeizing and irreducible parts. We expect such conditions to become especially helpful already in the the course of investigating the six-gluon amplitude. To illustrate the potential significance of the Ward identities let us briefly discuss the next step towards constructing the effectiv field theory, namely the investigation of the six-gluon amplitude $D_{6}$. In the first step, the quark loop offers sufficient inspiration for the sensible choice of a reggeizing part. However, in the six-gluon amplitude a new problem will arise. After decomposing the amplitude in the canonical way into reggeizing part and a remaining part, $D_{6}=D_{6}^{R}+D_{6}^{I}$, we will find a new integral equation for $D_{0}^{I}$. We anticipate that here a further decomposition will find a new integral equ contain a part that is the superposition of irreducible four-gluon amplitudes $D_{4}^{I}$

$$
D_{6}^{I}=D_{6}^{I, R}+D_{6}^{I, I}=\sum D_{4}^{I}+D_{6}^{I, I} .
$$

This means that the part $D_{6}^{I, R}$ is irreducible with respect to the two-gluon amplitude, but it is reducible with respect to the four-gluon amplitude. But in this case we do not have it is reducible with respect to the four-gluon amplitude. But in this case we do not have a quark loop suggesting a good choice for $D_{6}^{,, R}$. Exactly at this point the Ward identities
will be highly useful for the task of identifying a correct choice for the reggeizing part in this decomposition. To summarize, we expect roughly the following structure to arise in higher $n$-gluon amplitudes. There will most probably be irreducible $m$-gluon compound states for all even $m$. Based on each of them there will be a hierarchy of reggeizing parts of amplitudes, all of them reggeizing with respect to the same $m$-gluon compound state. The amplitudes in each of these hierarchies should then obey Ward type identities of the ind discused in this section. Unfortunately, at the moment we do not know a way to prove this conjecture at than to explicitly analyze the higher $n$-gluon amplitudes.

\subsection{Reggeization tensors in color space}

Whereas the preceding section was devoted to the study of the more global interplay of color and momentum structure in the amplitudes, we now turn to more local propertie f the color structure. Namely, we will be able to aseign to a rogeized gluon a lind of environment to study the mechanism of reggeization is clearly compound state or BFKL amplitude. To see how higher and higher 'Fock states' of the reggeized gluon occur we will therefore investigate the reggeizing parts $D_{n}^{R}$ of the $n$-gluon amplitudes that consist of superpositions of two-gluon amplitudes. Here we will focus ou interest on the color structure of single terms. For the most part the present section will deal with pure color algebra. One should therefore be very careful with the interpretation and not too quickly jump to conclusions that go substantially beyond the subject of colo structure. At the end of the section we will of course come back to the issue of interpreting the results in a larger context.

In general the process of reggeization can be viewed in two different ways. To illustrate this let us have a look at the reggeizing part $D_{4}^{R}$ of the four-gluon amplitude (3.3). In diagrammatic representation it is the first term in the right hand side of (3.16). In the picture of $t$-channel evolution the amplitudes start with the coupling of two reggeized gluons to the photons via a quark loop, then we have the propagation of the two-gluon state in the t-channel, and finally one of the gluons (or both) split - or 'decay' - into two or more gluons. To the splitting of the reggeized gluons belongs a certain color tensor, two or more gluons. To the splitting of the reggeized gluons belongs a certain color tensor,
as given in (3.3) for $D_{4}^{R}$. Viewed from a different angle, we can say that a group of gluons in the reggeizing part $D_{4}^{R}$ merges - or 'collapses' - to make up a more composite gluon which then enters the two-gluon compound state from below. We will use both pictures in parallel here and, depending on the context, speak of 'merging' or 'splitting' to mean the very same phenomenon of reggeization.

We will now turn to the case of arbitrary $n$ and consider the reggeizing parts $D_{n}^{R}$ of the amplitudes. From these we derive the color structure accompanying the merging of the a nut re of a number of roserar to a simple cles incation Thereby, we hope to gain furlier insight on how regsezation wons. However, we have to keep in mind that the amplitudes $D_{n}^{k}$ constitute only the simplest part of our amplitudes and are derived from the special structure of the quark loop. In a second step we will therfore have to find out whether the reggeization in more complex amplitudes like $D$ works in the same way, that is whether it is accompanied by the same color tensors as in the two-gluon amplitude. Only in this case we may speak of a general property of the mechanism of reggeization.

Let us pick one of the terms in the reggeizing part $D_{n}^{R}\left(\mathbf{k}_{1}, \ldots, \mathrm{k}_{n}\right)$. It consists of a two-gluon (BFKL) amplitude $D_{2}$ with its two arguments made up from a group of the $n$ mo-glon (B. and that the oller group is made of the resicity, we will further assume that the $l$ gluons in the fist group are the first $l$ gluons in the amplitude with momenta $\mathrm{k}_{1}, \ldots, \mathrm{k}_{l}$. Other terms in $D_{n}^{R}$ with a splitting into $l$ and $m$ gluons are then obtained by permutation of color and momentum labels. We can thus characterize the chosen term by its momentum structure,

$$
D_{2}(\underbrace{\mathbf{k}_{1}+\ldots+\mathbf{k}_{l}}_{l}, \underbrace{\mathbf{k}_{l+1}+\ldots+\mathbf{k}_{n}}_{m}) .
$$

In this section we will not care about the sign of the special term in $D_{n}^{R}$ we consider ${ }^{3}$. We will also neglect the additional factor $g^{n-2}$ that comes with the term above

To find the color tensor corresponding to (5.22) for arbitrary $n$ we have to remind ourselves of the way the reggeizing parts $D_{n}^{R}$ were constructed. The individual terms in $D_{n}^{R}$ were obtained by the replacement $D_{(2 ; 0)} \rightarrow D_{2}$ in the quark loop amplitude. The $D_{n}$ were obtained by the replacement $D_{(2 ; 0)} \rightarrow D_{2}$ in the fuart color tensor belonging to (5.22) can therefore be deduced from the corresponding lowest
order term in which $n$ gluons are coupled to the quark loop. Specifically, in the term of

${ }^{3}$ Especially in the case of an odd number of gluons, the relative signs of the terms in $D_{n}^{R}$ have to be treated with care since the signs change when the order of the color labels in the tensor is reversed. 
interest in the quark loop there are two contributions: one with the $l$ gluons of the first group coupled to the quark and the other $m$ gluons to the antiquark, the second with quark and antiquark exchanged. (This was described in more detail in section 2.4.) The trace in color space taken along the quark loop then gives for the first contribution

$$
\operatorname{tr}\left(t^{b_{1}} \ldots t^{b_{l}} t^{d_{m}} \ldots t^{d_{1}}\right)
$$

Here we have given new color labels to the gluons according to the group they are in. The first $l$ gluons now carry color labels $b_{i}$, the $m$ gluons in the second group have now been assigned the color labels $d_{j}$ such that the connection with the original labels is

$$
b_{i}=a_{i} \text { for } i \in\{1, \ldots, l\} ; \quad d_{j}=a_{l+j} \text { for } j \in\{1, \ldots, m\} .
$$

The second contribution contains a trace in color space in which the generators occur in reversed order,

$$
\operatorname{tr}\left(t^{d_{1}} \ldots t^{d_{1}} t^{b_{m}} \ldots t^{b_{1}}\right) .
$$

The relative sign between the two contributions depends on the total number $n$ of gluons. (This is because the coupling of a gluon to a quark or antiquark in the quark loop differ by a sign.) If $n$ is even, they come with the same sign. So the color tensor we are looking for is

$$
d^{b_{1} \ldots b_{l} d_{m} \ldots d_{1}}
$$

as defined in (2.33). If $n$ is odd, the two color traces in (5.23) and (5.25) come with opposite sign and we get a tensor of the form

$$
f^{b_{1} \ldots b_{l} d_{m} \ldots d_{1}}
$$

as it was defined in (2.34). It should be noted that in both cases the color labels of the one group come in ascending order in the tensor whereas those of the other group have to be taken in reversed order.

Now we make a little digression. It will turn out useful to have a look at the color structure arising from the successive emission of $l$ gluons off a quark. In color space this process is associated with

$$
t^{b_{1}} \ldots t^{b_{l}}=2 \operatorname{tr}\left(t^{b_{1}} \ldots t^{b_{l}} t^{c}\right) t^{c}+\frac{1}{N_{c}} \operatorname{tr}\left(t^{b_{1}} \ldots t^{b_{l}}\right) .
$$

The proof becomes almost obvious when we write this identity in birdtrack notation,

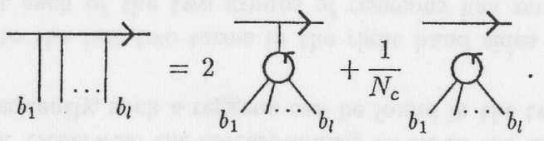

Applying the identity (2.31) to the right hand side we immediately find the left hand side. Using the definitions (2.33), (2.34) of $d$ and $f$ tensors we can rewrite this as

$$
t^{b_{1}} \ldots t^{b_{l}}=\left[d^{b_{1} \ldots b_{l} c}+i f^{b_{1} \ldots b_{l} c}\right] t^{c}+\frac{1}{2 N_{c}}\left[d^{b_{1} \ldots b_{l}}+i f^{b_{1} \ldots b_{l}}\right] \text {. }
$$

A special case of that general fact is the well-known formula describing the successive emission of two gluons off a quark,

$$
t^{a} t^{b}=\frac{1}{2}\left[\frac{1}{N_{c}} \delta_{a b}+\left(d_{a b c}+i f_{a b c}\right) t^{c}\right] .
$$

Here we have to have in mind $\mathrm{d}^{4}$ that the conventional normalization of structure constants $f_{a b c}$ and $d_{a b c}$ differs from our definition of $d$ - and $f$-tensors with upper indices. Further, we have to use $d^{a b}=\delta_{a b}$ and $f^{a b}=0$. A seemingly trivial special case of (5.30) is the emission of a single gluon. Namely, if $l=1$ only the first term on the right hand side give a contribution, and due to $d^{a b}=\delta_{a b}$ we end up with a trivial identity. Nevertheless, this case is quite important for the consistency of the assignment of 'quantum numbers' we want to carry out.

Coming back to our main problem now, we apply these identities to the color tensor of the term we have picked in $D_{n}^{R}$. The color tensor of that term depends on the total number $n$ of gluons. For even $n$ the color tensor associated with our term is the $d$-tensor in (5.26). Applying (5.30) now to the first group containing $l$ gluons we arrive at

$$
\begin{aligned}
d^{b_{1} \ldots b_{l} d_{m} \ldots d_{1}}= & d^{b_{1} \ldots b_{l} c} d^{c d_{1} \ldots d_{m}}+f^{b_{1} \ldots b_{1} c} f^{c d_{1} \ldots d_{m}} \\
& +\frac{1}{2 N_{c}} d^{b_{1} \ldots b_{l}} d^{d_{1} \ldots d_{m}}+\frac{1}{2 N_{c}} f^{b_{1} \ldots b_{1}} f^{d_{1} \ldots d_{m}}
\end{aligned}
$$

For odd $n$ the color tensor is the $f$-tensor in (5.27) and here the application of (5.30) to the $l$ gluons in the first group gives

$$
\begin{aligned}
f^{b_{1} \ldots b_{l} d_{m} \ldots d_{1}}= & -d^{b_{1} \ldots b_{l} c} f^{c d_{1} \ldots d_{m}}+f^{b_{1} \ldots b_{l} c} d^{c d_{1} \ldots d_{m}} \\
& -\frac{1}{2 N_{c}} d^{b_{1} \ldots b_{l}} f^{d_{1} \ldots d_{m}}+\frac{1}{2 N_{c}} f^{b_{1} \ldots b_{l}} d^{d_{1} \ldots d_{m}} .
\end{aligned}
$$

The relative signs again depend on the order of gluons we started with due to the definition of the $f$-tensor. We will not pay special attention to this detail here and concentrate on the tensors in the four terms separately.

These two decompositions of the $d$ - and $f$-tensors contain all possible combinations of even and odd $l$ and $m$, and the reggeization tensors we are looking for can now be extracted from the two decompositions. Obviously, the tensors describing the splitting in the two groups are correlated. We will now try to assign a kind of quantum number to the two reggeized gluons according to the way the respective reggeons split. This can only make sense if we demand that the two gluons in the two-reggeon state carry the same 'quantum number'. There will be four possible types of reggeons. We first want to fix the type of a reggeized gluon with color label $c$ that does not split. This case is included in the above identities as the splitting into one gluon $(l=1$ or $m=1)$ via a $d$-tensor (since we had $d^{a b}=\delta_{a b}$ ). We want to call this type a reggeized gluon of type in the adjoint representation. Now we can read off from the first term in (5.32) that such a reggeon decays into an odd number of gluons with a $d$-tensor. From the first or second term in (5.33) we find that it decays into an even number of gluons with a $f$-tensor. This assignment is consistent also if both $l>1$ and $m>1$.

Secondly, there is also the possibility that a reggeized gluon with color label $c$ splits into an even number of gluons via a $d$-tensor. We want to call such a reggeized gluon a 
reggeon of type $d$ in the adjoint representation. From the first lines in (5.32) and (5.33) we find consistently that such a reggeon splits into an odd number of gluons via a $f$-tensor. An interesting observation is that a reggeon of this type can only occur if it is composite of at least two gluons. Otherwise the corresponding terms in the above identities vanish due to $f^{a b}=0$. Consequently, such a reggeon can be found in the two-gluon state only if due to $f^{a b}=0$. Cons
both reggeons decay.

Now we proceed to the last two terms in the right hand sides of (5.32) and (5.33). Here we observe that each of the two groups of reggeons has zero total color charge. The corresponding composite reggeon is obviously in a color singlet state. This seems rather counter-intuitive. But our main focus in this chapter is the color structure and we therefore want to take the above identities seriously. Consequently, we have to treat these singlet reggeons on equal footing with the reggeons in the adjoint representation discussed before. Again, we can consistently define two different types, $d$ and $f$. A singlet reggeon of type $f$ splits into an even number of gluons via a $f$-tensor and into an odd number of gluons via a $d$-tensor. A $d$-type singlet reggeon decays into an even number of gluons via a $d$-tensor and into an odd number of gluons via a $f$-tensor. Here we do not have a certain decay mode we want to fix as of type $d$ or $f$ like in the case of the adjoint representation. Therefore in the assignment we could as well interchange $d$ and $f$. Like in the case of the $d$-type reggeon in the adjoint representation the singlet reggeons are composite of at least. two gluons. They cannot occur in a term in $D^{R}$ in which only one of the two regreized gluons in the two-gluon compound state decays.

We have been able to extract from the two color identities (5.32) and (5.33) in a consistent way a classification of reggeized gluons in the reggeizing amplitudes $D_{n}^{R}$. The classification is valid for arbitrary $n$ and all possible combinations of numbers $l$ and $m$ that merge into one the two gluons entering the two-gluon state. Let us surnmarize the assignments of reggeon types and the corresponding reggeization tensors in the table 5.1. $l$ denotes the number of reggeized gluons that merge into a more composite one. The diagrams for the color tensors are drawn with four and three legs here for illustration only. Of course they represent the tensors of type $d$ or $f$ for an arbitrary number $l$ of gluons

\begin{tabular}{|c|c|c|}
\hline reggeon type & $l$ even & $l$ odd \\
\hline$f$, adjoint rep. & & \\
\hline$d$, adjoint rep. & & \\
\hline$f$, singlet & & \\
\hline$d$, singlet & & R \\
\hline
\end{tabular}

Table 5.1: Reggeization tensors as obtained from the reggeizing parts $D_{n}^{R}$ as defined in (2.33) and (2.34). For $l \geq 3(l \geq 4$ for the singlet reggeons) the $d$ - and $f$-tensors in the table can be decomposed further into contractions of structure constants. The corresponding formulae for up to $l=5$ ( $l=6$ for the singlet reggeons) can be found in section 2.3.

In the case of $l=2$ the $d$-type reggeons can be interpreted as symmetric in the two gluons, and the $f$-type reggeons are antisymmetric in the two gluons. This holds in the adjoint representation as well as in the color singlet. However, this interpretation of $(f)$ as symmetric (antisymmetric) has to be refined for $l \geq 3$ since then the tensors are not completely (anti)symmetric in the $l$ color labels. Instead the symmetry of the $d$ - and $f$-tensors in individual pairs of gluons becomes more complicated.

In the reggeizing parts $D_{n}^{R}$ of the $n$-gluon amplitudes the different types of reggeons in our classification occur inevitably at the same time, since the tensors (5.32) and (5.33) contain them together. This is not necessarily the case in more complicated parts of the amplitudes that contain a compound state of more than two reggeons. For example, higher amplitudes will contain a part that does not reggeize with respect to the two-gluon higher amplitudes will contain a part that does not reggeize with respect to the two-gluon part $D_{5}^{I}$ of the five-gluon amplitude. It is well conceivable that in such amplitudes the part $D_{5}^{I}$ of the five-gluon amplitude. It is well conceivable that in such amplitudes the
four reggeons in the compound state are less correlated than in the two-gluon state and four reggeons in the compound state are le
the reggeon types can occur independently.

The example of the part $D_{5}^{I}$ of the five-gluon is, however, not sufficient to clarify the
The regen types can ocur indepently. situation. On the one hand, it confirms our classification: the four gluons in $D_{4}^{I}$ are of type $f$ in the adjoint representation and one of them in fact splits into two with the tensor that should be expected from our classification. On the other hand, we do not expect the other types of reggens to appear in $D$ If our classfication is ight, since thos require two splittings in the whole amplitude. We thus bave to go at least to the six-gluon amplitude to study their behavior.

In this respect the $f$-type reggeon in the adjoint representation plays a special role. It is the only type of reggeon that occurs in $D_{n}^{R}$ when only one of the two reggeons decays, i. e. when the other one splits trivially into one gluon. This observation leads us to suspect that the $f$-type reggeon in the adjoint representation can appear in each possible compound state. In the next chapter we will investigate the six-gluon amplitude and find hints that agree with this conjecture. For the other types the correlation of the two reggeons in the two-gluon compound state seems to be essential. It seems natural the expect that their behavior in higher compound states is more complicated. Most probably, the knowber of the regreizing parts $D^{R}$ is not sufficit to fully understand these types. This will also be confirmed by our investigation of the six-gluon amplitude in the next chapter.

At present, the classification developed in this section has the status of an observation. We are not able to derive the decay tensors from first principles for any reggeon in any amplitude. This would probably require a different approach. Possibly an effective action framework $[39,40]$ offers a suitable starting point. Certainly, our findings have to be tested in the investigation of higher $n$-gluon amplitudes. Especially the universality of the concept outlined here is by no means obvious.

There is certainly a deeper connection with the notion of signature to be discovered here. The reggeon of type $f$ in the adjoint representation can be identified as a reggeized gluon with the usual negative signature. The reggeon of type $d$ in the adjoint representation can probably be identified with a reggeized gluon carrying positive signature. For the singlet reggeons the situation is not so clear. It will of course be very interesting to 
un derstand this relation to signature also in the other cases. However, at present it seems a bit too early to draw final conclusions. Before that the universality of the reggeization tensors should be investigated. In addition the somewhat confusing situation with the singlet reggeons should be clarified by finding a consistent interpretation for them.

\section{Chapter 6}

\section{Six Gluons}

In this chapter we turn our attention towards the six-gluon amplitude. We hope to find answers to several questions by studying this amplitude. We expect that a transition from two to six gluons can happen in two steps by successive two-to-four transitions. Since a pair of gluons in the irreducible amplitude $D_{4}^{I}$ is not necessarily in a color singlet state the second step in that transition will provide information on the two-to-four vertex in the color non-singlet. It will also be interesting to find out if there is a direct transition from two to six gluons, i.e. a new number-changing element of the effective field theory. To find out about these questions will certainly require to understand the mechanism of reggeization in the four-gluon state, as has been indicated already in the preceding chapters. We will therer 5 to the test. In addition, the six-gluon amplitude offers the possibility of studying a possible Pomeron-Odderon-Odderon vertex

We use our tried and tested method to attack the integral equation for the six-gluon amplitude. A reggeizing part is identified in section 6.1 , and a new integral equation for the remaining part of the six-gluon amplitude is derived in section 6.2 where we also discuss its properties. A new piece is discovered in the equation that cannot be expressed in terms of the known two-to-four vertex. This piece is the subject of section 6.3 where we discuss its properties and prove its conformal invariance. We speculate on its role in the effective field theory. In section 6.4 we try to identify a part of the six-gluon amplitude that reggeizes with respect to the four-gluon state. Such a part would permit to apply our procedure for dealing with the integral equations to the new integral equation found in section 6.2. Unfortunately, the structure of that integral equation is rather intricate. We encounter technical as well as conceptual problems which presently prevent us from finding conclusive answers to the questions raised above. We speculate on possible ways to resolve the occurring problems. In section 6.5 we investigate the possibility of a PomeronOdderon-Odderon vertex and in fact find contributions to such a vertex in the integral equation derived in section 6.2. Finally, we discuss the results obtained in this chapter in the light of an effective field theory of unitarity corrections.

\subsection{A reggeizing part}

Encouraged by the success we have had so far with that procedure we again use the quar loop amplitude $D_{(6 ; 0)}$ to construct from it a reggeizing part $D_{6}^{R}$ as a superposition of 
two-gluon (BFKL) amplitudes. The full six-gluon amplitude is then split into two parts

$$
D_{6}=D_{6}^{R}+D_{6}^{I},
$$

and it will be our first task to find a new integral equation for the remaining part $D_{6}^{I}$. In detail, the reggeizing part $D_{6}^{R}$ is

$$
\begin{aligned}
D_{6}^{R a_{1} a_{2} a_{3} a_{4} a_{5} a_{6}}\left(\mathrm{k}_{1}, \mathrm{k}_{2}, \mathrm{k}_{3}, \mathrm{k}_{4}, \mathrm{k}_{5}, \mathrm{k}_{6}\right)= \\
=\quad g^{4}\left\{d^{a_{1} a_{2} a_{3} a_{4} a_{5} a_{6}}\left[D_{2}(12345,6)+D_{2}(1,23456)-D_{2}(16,2345)\right]\right. \\
\quad+d^{a_{2} a_{1} a_{3} a_{4} a_{5} a_{6}}\left[D_{2}(13456,2)-D_{2}(1345,26)+D_{2}(126,345)-D_{2}(12,3456)\right] \\
\quad+d^{a_{1} a_{2} a_{3} a_{4} a_{6} a_{5}}\left[D_{2}(12346,5)-D_{2}(1234,56)+D_{2}(156,234)-D_{2}(15,2346)\right] \\
\quad+d^{a_{2} a_{1} a_{3} a_{4} a_{6} a_{5}}\left[-D_{2}(1256,34)-D_{2}(1346,25)+D_{2}(125,346)+D_{2}(134,256)\right] \\
\quad+d^{a_{3} a_{1} a_{2} a_{4} a_{5} a_{6}}\left[D_{2}(12456,3)-D_{2}(1245,36)+D_{2}(136,245)-D_{2}(13,2456)\right] \\
\quad+d^{a_{2} a_{2} a_{3} a_{5} a_{6} a_{4}}\left[D_{2}(12356,4)-D_{2}(1235,46)+D_{2}(146,235)-D_{2}(14,2356)\right] \\
+ \\
+d^{a_{2} a_{1} a_{3} a_{5} a_{6} a_{4}}\left[-D_{2}(1246,35)-D_{2}(1356,24)+D_{2}(124,356)+D_{2}(135,246)\right] \\
+d^{a_{1} a_{2} a_{3} a_{6} a_{5} a_{4}}
\end{aligned}
$$

as obtained from (2.67) by the replacement $D_{(2 ; 0)} \rightarrow D_{2}$ while keeping the color and momentum structure. This expression already indicates one of the major difficulties we have to overcome during the treatment of the six-gluon amplitude: the large number of terms we have to take care of.

\subsection{The integral equation for the remaining part}

The original integral equation (2.15) for the six-gluon amplitude is now used to derive a new integral for the unknown part $D_{6}^{I}$. The method in this step is exactly the same as for the four- and five-gluon amplitudes. We insert into the integral equation our complete knowledge on the reggeizing parts $D_{n}^{R}$ of the amplitudes $D_{n}$ with up to $n=6$ gluons, including the ansatz (6.1),(6.2) for the six-gluon amplitude. The corresponding formulae for $n \leq 5$ can be found in the preceding chapters. Then we apply the BFKL equation (2.11) to the expression $\omega D_{6}^{R}$ on the left hand side. This is possible because $D_{6}^{R}$ was chosen as a superposition of BFKL amplitudes, cf. (6.2). We thereby produce convolutions of $D_{2}$ amplitudes with two-to-two kernels and products of $D_{2}$ amplitudes with trajectory functions $\beta$. The insertion of the reggeizing parts $D_{n}^{R}$ of the amplitudes on the right hand side leads to convolutions of $D_{2}$ amplitudes with the integral kernels. the have to perform the hals to their st the huge number of combinations of amplitudes with kernels. We have to perform close to 250 contractions of color tensors, and we have to find the standard form of more than 3500 integrals. Whereas the color tensors can still be calculated by hand this is no longer possible for the huge number of momentum space integrals. We have therefore developed an algorithm for this purpose that is suited for the implementation on a computer. The algorithm is explained in detail in appendix B. We have written a PERL script based on this algorithm that produces an output which can directly be used as an input for a computer algebra program like MAPLE. The tensor contractions are calculated with the help of the method described in appendix A. Some of the contractions are given explicitly in that appendix. Many other contractions are obtained from these by permutations of the gluon color labels. The computer algebra program is then used to multiply the resulting sums of elementary tensors with the corresponding integrals, and to finally collect all terms. In the final step more than $2 \cdot 10^{4}$ integrals have to be sorted according to their color tenso coefficients. (This shows that our method of dealing with the integrat equations will in (n) relatively small numbers $n$ of gluons.) Havin collected all terms in the equation which contain the amplitude $D_{2}$ we have found th homogeneous term of the new integral equation for $D_{6}^{1}$

In the derivation of the new integral equation the terms containing the irreducible four-gluon amplitude $D_{4}^{I}$ and the second part $D_{5}^{I}$ of the five-gluon amplitude remain unchanged. Their combinations with the kernels are the same as in the original equation (2.15). The resulting integral equation for $D_{6}^{I}$ is then found to have the form

$$
\begin{aligned}
(\omega & \left.-\sum_{i=1}^{6} \beta\left(\mathrm{k}_{i}\right)\right) D_{6}^{I a_{1} a_{2} a_{3} a_{4} a_{5} a_{6}}\left(\mathbf{k}_{1}, \mathbf{k}_{2}, \mathbf{k}_{3}, \mathbf{k}_{4}, \mathbf{k}_{5} \mathbf{k}_{6}\right)= \\
= & \left(W^{a_{1} a_{2} a_{3} a_{4} a_{5} a_{6}} D_{2}\right)\left(\mathbf{k}_{1}, \mathbf{k}_{2}, \mathbf{k}_{3}, \mathbf{k}_{4}, \mathbf{k}_{5} \mathrm{k}_{6}\right) \\
& +\sum f_{a_{1} a_{2} a_{3}} f_{a_{4} a_{5} a_{6}} L(1,2,3 ; 4,5,6) \\
& +\sum d^{a_{1} a_{2} a_{3} a_{4}} \delta_{a_{5} a_{6}} I(1,2,3,4 ; 5,6) \\
& +\sum d^{a_{2} a_{1} a_{3} a_{4}} \delta_{a_{5} a_{6}} J(1,2,3,4 ; 5,6) \\
& +\sum K_{2 \rightarrow 4}^{\{b\} \rightarrow\{a\}} \otimes D_{4}^{I b_{1} b_{2} b_{3} b_{4}}+\sum K_{2 \rightarrow 3}^{\{b\} \rightarrow\{a\}} \otimes D_{5}^{I b_{1} b_{2} b_{3} b_{4} b_{5}} \\
& +\sum K_{2 \rightarrow 2}^{\{b\} \rightarrow\{a\}} \otimes D_{6}^{I b_{1} b_{2} b_{3} b_{4} b_{5} b_{6}} .
\end{aligned}
$$

The first four terms on the right hand side are the result of the computation outlined above. We will now describe them in detail.

The first observation we make is again that certain color structures are completely cancelled in the equation. All terms proportional to $d^{a_{1} a_{2} a_{3} a_{4} a_{5} a_{6}}$ (and the other seven permutations of this occurring in (6.2)) are cancelled between the different contributions to the inhomogeneous term and drop out. The same mechanism was observed in the equations for the parts $D_{4}^{I}$ and $D_{5}^{I}$ of the four-and five-gluon amplitudes for the tensor $d^{a_{1} a_{2} a_{3} a_{4}}$ and $f^{a_{1} a_{2} a_{3} a_{4} a_{5}}$ respectively.

As in the integral equations for $D_{4}^{I}$ and $D_{5}^{I}$ the inhomogeneous term has a high degree of symmetry which we will explain for each of the terms below. This symmetry is not only nice by itself, but it is also a highly welcome possibility to check our calculation.

The first term on the right hand side of (6.3) differs in its structure from the other terms and will be treated separately in section 6.3 . Here we mention already that it is symmetric in the sense that it is the sum of terms that are obtained from each other by permutations of the gluons.

The same is true for the second term on the right hand side of the new integral equation. The sum extends over all partitions of the six gluons into two groups each of which contains three gluons,

$$
\begin{aligned}
\sum f_{a_{1} a_{2} a_{3}} f_{a_{4} a_{5} a_{6}} L(1,2,3 ; 4,5,6)= & f_{a_{1} a_{2} a_{3}} f_{a_{4} a_{5} a_{6}} L(1,2,3 ; 4,5,6) \\
& +f_{a_{1} a_{2} a_{4}} f_{a_{3} a_{5} a_{6}} L(1,2,4 ; 3,5,6) \\
& +\ldots+f_{a_{1} a_{5} a_{6}} f_{a_{2} a_{3} a_{4}} L(1,5,6 ; 2,3,4) .
\end{aligned}
$$


The function $L$ is the same in all terms in the sum and only its arguments are exchanged in the different terms. A closer inspection reveals that the function $L$ permits a decomposition into vertex functions $V$ known from the two-to-four transition vertex (see chapter 3),

$$
\begin{aligned}
L(1,2,3 ; 4,5,6)=\frac{g^{2}}{4} & {\left[\left(V D_{2}\right)(12,3 ; 45,6)-\left(V D_{2}\right)(12,3 ; 46,5)+\left(V D_{2}\right)(12,3 ; 4,56)\right.} \\
& -\left(V D_{2}\right)(13,2 ; 45,6)+\left(V D_{2}\right)(13,2 ; 46,5)-\left(V D_{2}\right)(13,2 ; 4,56) \\
& +\left(V D_{2}\right)(1,23 ; 45,6)-\left(V D_{2}\right)(1,23 ; 46,5) \\
& \left.+\left(V D_{2}\right)(1,23 ; 4,56)\right] .
\end{aligned}
$$

The sum in the third term on the right hand side of the integral equation extends over all partitions of the six gluons into one group containing four and one group containing two gluons,

$$
\begin{aligned}
\sum d^{a_{1} a_{2} a_{3} a_{4}} \delta_{a_{5} a_{6}} I(1,2,3,4 ; 5,6)= & d^{a_{1} a_{2} a_{3} a_{4}} \delta_{a_{5} a_{6}} I(1,2,3,4 ; 5,6) \\
& +d^{a_{1} a_{2} a_{3} a_{5}} \delta_{a_{4} a_{6}} I(1,2,3,5 ; 4,6)
\end{aligned}
$$$$
+\ldots+\delta_{a_{1} a_{2}} d^{a_{3} a_{4} a_{5} a_{6}} I(3,4,5,6 ; 1,2) . \quad(6.6)
$$

Also in this case we find that the function $I$ is the same in all terms in the sum. Remarkably, also this function can be written in terms of the vertex function $V$,

$I(1,2,3,4 ; 5,6)=-g^{2}\left[\left(V D_{2}\right)(1,234 ; 5,6)+\left(V D_{2}\right)(123,4 ; 5,6)-\left(V D_{2}\right)(14,23 ; 5,6)\right]$.

The sum in the fourth term on the right hand side of the new integral equation (6.3),

$$
\sum d^{a_{2} a_{1} a_{3} a_{4}} \delta_{a_{5} a_{6}} J(1,2,3,4 ; 5,6),
$$

extends over the same permutations of gluons as the term discussed before (see (6.6)). Again the function $J$ is the same in all terms in the sum, and it can be written as a superposition of vertex functions $V$ as

$$
\begin{array}{r}
J(1,2,3,4 ; 5,6)=-g^{2}\left[\left(V D_{2}\right)(134,2 ; 5,6)+\left(V D_{2}\right)(124,3 ; 5,6)\right. \\
\left.-\left(V D_{2}\right)(12,34 ; 5,6)-\left(V D_{2}\right)(13,24 ; 5,6)\right] .
\end{array}
$$

We would like to emphasize that the symmetry of the sums contributing to the inhomogeneous term of the new integral equation is an outcome of our calculation. We have not used it to derive the new equation. That we find the symmetry in the resulting equation gives us confidence that we did not make any errors in the long and tedious calculation leading to (6.3). We also would like to stress that the representation of a part of the inhomogeneous term as a superposition of well-known vertex function $V$ is an outcome of our calculation and was not used to derive the new equation. Unfortunately, we do not know a way that directly leads to the comparatively simple structure arising in the terms discussed above.

Before coming back to the terms in the equation that can be decomposed into vertex functions $V$ we discuss in the following section the first term on the right hand side of equation (6.3).

\subsection{A new piece in the field theory}

The first term on the right hand side of the integral equation (6.3) differs in its structure from the other terms. We will therefore discuss it separately in this section. The study of the other terms will be resumed in section 6.4. We start by giving an explicit representation of the term under consideration and proceed by listing its properties. After that we speculate on the place the new term might find in the effective field theory of unitarity corrections.

\subsubsection{Explicit representation and properties}

The first term on the right hand side in (6.3) has the following color and momentum structure:

$\left(W^{a_{1} a_{2} a_{3} a_{4} a_{5} a_{6}} D_{2}\right)\left(\mathbf{k}_{1}, \mathbf{k}_{2}, \mathbf{k}_{3}, \mathbf{k}_{4}, \mathbf{k}_{5}, \mathbf{k}_{6}\right)=\sum d_{a_{1} a_{2} a_{3}} d_{a_{4} a_{5} a_{6}}\left(W D_{2}\right)(1,2,3 ; 4,5,6)$.

The sum extends over all (ten) partitions of the six gluons into two groups collo three gluons each,

$$
\begin{aligned}
\sum d_{a_{1} a_{2} a_{3}} d_{a_{4} a_{5} a_{6}}\left(W D_{2}\right)(1,2,3 ; 4,5,6)= & d_{a_{1} a_{2} a_{3}} d_{a_{a_{1}} a_{5}}\left(W D_{2}\right)(1,2,3 ; 4,5,6) \\
& +d_{a_{1} a_{2} a_{4}} d_{a_{3} a_{5} a_{6}}\left(W D_{2}\right)(1,2,4 ; 3,5,6)+\ldots \\
& +d_{a_{1} a_{5} a_{6}} d_{a_{2} a_{3} a_{4}}\left(W D_{2}\right)(1,5,6 ; 2,3,4) \cdot(6.11)
\end{aligned}
$$

The function $W D_{2}$ is the same in all permutations. (Again this is an outcome of our calculation and was not assumed at any stage when the equation (6.3) was derived.) The sum thus contains the same permutations of the six gluons as the second term (6.4) on the right hand side of (6.3). For the notation to be consistent the function $W^{a_{1} a_{2} a_{3} a_{4} a_{5} a_{6}}$ should be understood as an integral operator acting on a BFKL amplitude $D_{2}$. It thus carries two more momentum arguments $\mathbf{q}_{j}$ for the momenta entering from above. We will again suppress these two momenta in the following.

In contrast to the other terms in (6.3) discussed so far the function $W$ does not permit a decomposition into the vertex functions $V$ known from the two-to-four transition vertex. We therefore give its full momentum space representation as we have obtained it as a result of our calculation. We again use the standard integrals defined in section 2.7. Then $W D_{2}$ has the explicit representation

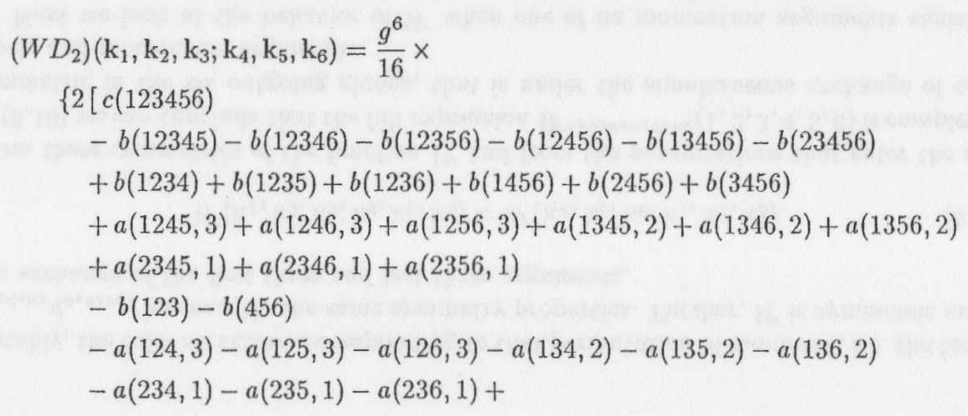


$-a(145,23)-a(146,23)-a(156,23)-a(245,13)-a(246,13)-a(256,13)$ $-a(345,12)-a(346,12)-a(356,12)$

$+a(12,3)+a(13,2)+a(23,1)+a(45,123)+a(46,123)+a(56,123)$ $+a(14,23)+a(15,23)+a(16,23)+a(24,13)+a(25,13)+a(26,13)$ $+a(34,12)+a(35,12)+a(36,12)$

$-a(1,23)-a(2,13)-a(3,12)-a(4,123)-a(5,123)-a(6,123)]$

$+[t(12345)+t(12346)+t(12356)+t(12456)+t(13456)+t(23456)$

$-t(1234)-t(1235)-t(1236)-t(1456)-t(2456)-t(3456)$

$-s(1245,3)-s(1245,6)-s(1246,3)-s(1246,5)-s(1256,3)-s(1256,4)$

$-s(1345,2)-s(1345,6)-s(1346,2)-s(1346,5)-s(1356,2)-s(1356,4)$

$-s(2345,1)-s(2345,6)-s(2346,1)-s(2346,5)-s(2356,1)-s(2356,4)$

$+t(123)+t(456)$

$+s(124,3)+s(124,56)+s(125,3)+s(125,46)+s(126,3)+s(126,45)$

$+s(134,2)+s(134,56)+s(135,2)+s(135,46)+s(136,2)+s(136,45)$

$+s(234,1)+s(234,56)+s(235,1)+s(235,46)+s(236,1)+s(236,45)$

$+s(145,23)+s(145,6)+s(146,23)+s(146,5)+s(156,23)+s(156,4)$

$+s(245,13)+s(245,6)+s(246,13)+s(246,5)+s(256,13)+s(256,4)$

$+s(345,12)+s(345,6)+s(346,12)+s(346,5)+s(356,12)+s(356,4)$

$-s(12,3)-s(12,456)-s(13,2)-s(13,456)-s(23,1)-s(23,456)$

$-s(45,123)-s(45,6)-s(46,123)-s(46,5)-s(56,123)-s(56,4)$

$-s(14,23)-s(14,56)-s(15,23)-s(15,46)-s(16,23)-s(16,45)$

$-s(24,13)-s(24,56)-s(25,13)-s(25,46)-s(26,13)-s(26,45)$

$-s(34,12)-s(34,56)-s(35,12)-s(35,46)-s(36,12)-s(36,45)$

$+s(1,23)+s(1,456)+s(2,13)+s(2,456)+s(3,12)+s(3,456)$

$+s(4,123)+s(4,56)+s(5,123)+s(5,46)+s(6,123)+s(6,45)]\}$.

On first sight this expression appears to be very complicated. Closer inspection reveals that it has a series of very interesting properties. Some of them very much resemble those of the function $V$ we have described in sections 3.2 and 3.4. We will now study these properties. At $V$ wection 6.3 .2 we will elaborate on the qustion how the new pice $(6.10)$ At subsetion 6.3 .2 we will elabote on the has to be interpreted in the context of the effective field theory structure of unitarity

Let us first have a look at the symmetry properties of the function $W$. We find that $W$ is fully symmetric in its first three arguments

$$
W\left(\mathbf{k}_{1}, \mathbf{k}_{2}, \mathbf{k}_{3} ; \mathbf{k}_{4}, \mathrm{k}_{5}, \mathrm{k}_{6}\right)=W\left(\mathrm{k}_{2}, \mathrm{k}_{1}, \mathrm{k}_{3} ; \mathbf{k}_{4}, \mathrm{k}_{5}, \mathrm{k}_{6}\right)
$$

$=W\left(\mathrm{k}_{3}, \mathrm{k}_{2}, \mathrm{k}_{1} ; \mathrm{k}_{4}, \mathrm{k}_{5}, \mathrm{k}_{6}\right)$

as well as in its last three arguments,

$W\left(\mathrm{k}_{1}, \mathrm{k}_{2}, \mathrm{k}_{3} ; \mathrm{k}_{\mathbf{4}}, \mathrm{k}_{5}, \mathrm{k}_{6}\right)=W\left(\mathrm{k}_{1}, \mathrm{k}_{2}, \mathrm{k}_{3} ; \mathrm{k}_{5}, \mathrm{k}_{\mathbf{4}}, \mathrm{k}_{6}\right)$

$=W\left(\mathbf{k}_{1}, \mathbf{k}_{2}, \mathbf{k}_{3} ; \mathbf{k}_{6}, \mathbf{k}_{5}, \mathbf{k}_{4}\right)$.
Notably, the color structure corresponding to that permutation of momenta, i. e. the tensor $d_{a_{1} a_{2} a_{3}} d_{a_{4} a_{5} a_{6}}$, has exactly the same symmetry properties. Further, $W$ is symmetric under the exchange of the first three and last three arguments,

$$
W\left(\mathrm{k}_{1}, \mathrm{k}_{2}, \mathrm{k}_{3} ; \mathrm{k}_{4}, \mathrm{k}_{5}, \mathrm{k}_{6}\right)=W\left(\mathrm{k}_{4}, \mathrm{k}_{5}, \mathrm{k}_{6} ; \mathrm{k}_{1}, \mathrm{k}_{2}, \mathrm{k}_{3}\right) .
$$

From these symmetries of the function $W$ and from the permutations that enter the sum in (6.10) we can conclude that the full expression $W^{a_{1} a_{2} a_{3} a_{4} a_{5} a_{6}}(1,2,3,4,5,6)$ is completely symmetric in the six outgoing gluons, that is under the simultaneous exchange of color labels and momentum arguments.

Next we look at the behavior of $W$ when one of its momentum arguments vanishes. Not unexpectedly, we find that $W$ vanishes whenever one of the six gluons carries zero transverse momentum,

$$
\left.W\left(\mathrm{k}_{1}, \mathrm{k}_{2}, \mathrm{k}_{3} ; \mathrm{k}_{4}, \mathrm{k}_{5}, \mathbf{k}_{6}\right)\right|_{\mathbf{k}_{i}=0}=0 \quad(i \in\{1, \ldots, 6\}) .
$$

Starting from the explicit representation (6.12) the proof is straightforward. Of course, we again have to use the relations between the different standard integrals mentioned at the end of section 2.7 and the fact that the gluon trajectory function $\beta(\mathbf{k})$ vanishes for $\mathrm{k}=0$.

Further we find that the function $W$ is infrared finite. The proof just requires a close inspection of the standard integrals occurring in (6.12). It is easily seen that the integrals come in the infrared combinations (3.11) and (3.12) discussed already in section 3.2. (Again, the integrals in (6.12) have been arranged in a way that hopefully makes this 3.2. (Again,
transparent.)

The function $W$ cannot be decomposed into a superposition of vertex functions $V$ known from the two-to-four vertex. But it turns out that a decomposition into a sum of $G$-functions (3.21) is possible,

$$
\left(W D_{2}\right)\left(\mathrm{k}_{1}, \mathrm{k}_{2}, \mathrm{k}_{3} ; \mathrm{k}_{4}, \mathrm{k}_{5}, \mathrm{k}_{6}\right)=\frac{g^{4}}{8} \times
$$

$$
[G(123,-, 456)
$$

$-G(12,3,456)-G(13,2,456)-G(23,1,456)$

$-G(123,4,56)-G(123,5,46)-G(123,6,45)$

$+G(1,23,456)+G(2,13,456)+G(3,12,456)$

$+G(123,45,6)+G(123,46,5)+G(123,56,4)$

$+G(12,34,56)+G(13,24,56)+G(23,14,56)$

$+G(12,35,46)+G(13,25,46)+G(23,15,46)$ $+G(12,36,45)+G(13,26,45)+G(23,16,45)$ - $G(1,234,56)-G(2,134,56)-G(3,124,56)$ $-G(1,235,46)-G(2,135,46)-G(3,125,46)$ $-G(1,236,45)-G(2,136,45)-G(3,126,45)$ $-G(12,345,6)-G(12,346,5)-G(12,356,4)$ - $G(13,245,6)-G(13,246,5)-G(13,256,4)$ $-G(23,145,6)-G(23,146,5)-G(23,156,4)+$ 
$+G(1,2345,6)+G(2,1345,6)+G(3,1245,6)$

$+G(1,2346,5)+G(2,1346,5)+G(3,1246,5)$

$+G(1,2356,4)+G(2,1356,4)+G(3,1256,4)]$

This decomposition has a very strong implication. The function $G$ is conformally invariant in two-dimensional impact parameter space (see section 3.4 ). Therefore, the decomposition (6.17) immediately proves the conformal invariance of the function $W$ in impact parameter space.

It is worth noting that all the properties of the new piece (6.10) have also been found in the two-to-four transition vertex $V_{2 \rightarrow 4}$ (see chapter 3). A closer look reveals that even the construction of the functions $V$ and $W$ in (3.6) and (6.12) follows the same scheme.

\subsubsection{Interpretation in view of an effective field theory}

It is now natural to ask where the new piece $\left(W D_{2}\right)^{a_{1} a_{2} a_{3} a_{4} a_{5} a_{6}}$ finds its place in the effective field theory of unitarity corrections. As we have seen in the preceding section the new piece has properties that very much resemble those of the two-to-four transition vertex $V_{2 \rightarrow 4}$. It is fully symmetric in the six gluons, it is infrared finite, and it vanishes when one of the gluon momenta vanishes. In addition, it is conformally invariant in impact parameter space. It seems to be perfectly obvious that the new piece $W^{a_{1} a_{2} a_{3} a_{4} a_{5} a_{6}}$ is a new two-to-six gluon transition vertex $V_{2 \rightarrow 6}$ and that it constitutes a new element of the effelive fell thery. three gluons and in the remaining three gluons. Then the function $W$ is split into several parts according to the symmetry or antisymmetry under the exchange of the gluons 1 and 2 , say, and under the exchange of the pair (12) of gluons with gluon 3 , and analogously for the other three gluons. Based on the result of this procedure it is possible to define new two-to-four vertices with symmetry properties different from those of the well-known two-to-four vertex $V_{2 \rightarrow 4}$. Based on these one can in turn define new four-gluon amplitudes that then become basic elements of the effective feld theory. Due to this a direct tranition fron two to ix regreied gluons is avidel. This . Fin ally, the is a thind in vestigated. Finally, there is a third possibility. As we will see in the following section, a problem occurs when we try to understand certain contributions to the other terms in the new integral equation (6.3). It is conceivable that the resolution of that problem might possibly bring about an 'interference' with the term under consideration in this section. This is very unlikely since the other terms can be written as superpositions of the wellknown vertex functions $V$ whereas this is not possible for the function $W$. Nevertheless, this third possibility can at present not be excluded.

To conclude this section, I can say that the interpretation of the new piece as a two-tosix gluon transition vertex is certainly very tempting. But the other possibilities are still viable and deserve further study before a final interpretation of the new piece is possible.

\subsection{Further reggeization}

Now we come to discuss the other terms that are present in the new integral equation (6.3) for the part $D_{6}^{I}$ of the six-gluon amplitude. In section 6.2 we have already shown that these term can be written as superpositions of well-known vertex functions $V$. We will in this section disregard the new piece discussed in the previous section.

In chapter 4 we already encountered a situation similar to the one which we find here in the integral equation (6.3). Also there the inhomogeneous term of the integral equation for $D_{5}^{I}$ could be written as a superposition of vertex functions $V$. It was characteristic indication for the occurrence of a further reggeization of the amplitude $D_{5}^{I}$ with respect to the irreducible four-gluon amplitude $D_{4}^{I}$. This idea even allowed us to find the exact solution of the equation. We cannot expect that the remaining part $D_{6}^{I}$ of the six-gluon amplitude reggeizes completely. But the occurrence of the vertex functions in the inhomogeneous term of its equation strongly suggests that a part of $D_{6}^{I}$ will reggeize. To gain further insight we should therefore construct an ansatz for the remaining part in order to simplify the integral equation (6.3). The remaining part $D_{6}^{I}$ should thus be split into a reggeizing part and an irreducible part,

$$
D_{6}^{I}=D_{6}^{I, R}+D_{6}^{I, I},
$$

where this time the term 'reggeizing' refers to the reggeization with respect to the fourgluon compound state, cf. the discussion at the end of sections 4.3 and 5.1. The reggeizing part should be a superposition of irreducible four-gluon amplitudes, symbolically

$$
D_{6}^{I, R}=\sum D_{4}^{I} .
$$

The problem is now to find the correct color and momentum structure for the right hand side of this symbolic equation.

We should have in mind that the inhomogeneous term in the integral equations for $D_{n}$, that is the quark loop, is always an excellent source of inspiration for the choice of a reggeizing part $D_{n}^{R}$. To make a good guess for the reggeizing part $D_{6}^{I, R}$ we should therefore have a close look at the inhomogeneous term of the new integral equation (6.3)

Let us first look at the terms (6.7) and (6.9) containing the functions $I$ and $J$. We will pick one permutation in the sums (6.6) and (6.8) only, the other permutations can then be treated in analogy. We see immediately that the color and momentum structure in the first four gluons in the terms

$$
d^{a_{1} a_{2} a_{3} a_{4}} \delta_{a_{5} a_{6}} I(1,2,3,4 ; 5,6)
$$

and

$$
d^{a_{2} a_{1} a_{3} a_{4}} \delta_{a_{5} a_{6}} J(1,2,3,4 ; 5,6)
$$

is exactly the same that we know from $D_{4}^{R}$.

A second observation we make is a certain mismatch between $f_{a b c}$ and $d_{a b c}$ tensors. Whereas there are terms of the kind

$$
\sum f_{a_{1} a_{2} a_{3}} f_{a_{4} a_{5} a_{8}} L(1,2,3 ; 4,5,6)
$$

present in the equation which can be written in terms of $V$ the corresponding terms with $d_{a b c}$ tensors (the new piece, see section 6.3) cannot be written in a similar way. This already indicates that the $f$ - and $d$-tensors have to be treated differently.

In order to come from an ansatz of the form (6.19) to the cancellation of the inhomogeneous term in the integral equation we have to use the integral equation (3.4) for the 
irreducible part $D_{4}^{I}$ of the four-gluon amplitude. We want to write the arguments of the $D_{4}^{I}$ 's in the ansatz in such a way that they have exactly the same momentum structure which we find in the vertex functions $V$ in (6.3). This is in complete analogy to the parts $D_{n}^{R}$ in which the momentum structure was taken from the quark loop. In the case of $D_{n}^{R}$ we could also keep the color structure. This was possible because the color structure of the two-gluon amplitude $D_{2}$ was trivial, i.e. the two gluons were always in a color singlet state. That allowed to factorize the two-gluon amplitude into a color part $\left(\delta_{a_{1} a_{2}}\right)$ and a momentum part. Now the situation is more complicated since such a factorization is not possible for the irreducible four-gluon amplitude. We have to use the full amplitude $D_{4}^{I b_{1} b_{2} b_{3} b_{4}}$. Since we certainly need the six color labels $a_{1}, \ldots, a_{6}$ in the ansatz $(6.19)$, the mathematical procedure we have to use is the contraction with a tensor $\Theta$,

$$
\Theta^{a_{1} a_{2} a_{3} a_{4} a_{5} a_{6} ; b_{1} b_{2} b_{3} b_{4}} D_{4}^{I b_{1} b_{2} b_{3} b_{4}} .
$$

The tensor $\Theta$ is an invariant tensor in the ten-fold tensor product $\otimes_{i=1}^{10}\left[s u\left(N_{c}\right)\right]$ of the Lie algebra. It will obviously be very difficult to find the correct tensors for the contractions in this huge tensor space. We have to hope that the situation is in a certain sense more simple. It will be necessary to find restrictions on the tensors from the inhomogeneous term in the new integral equation. Now a problem arises. In the term

$$
d^{a_{1} a_{2} a_{3} a_{4}} \delta_{a_{5} a_{6}} V(123,4 ; 5,6)
$$

for example the first four gluons are in an overall color singlet state, as are the last two gluons. Obviously, the color tensor necessary for the contraction with $D_{4}^{I b_{1} b_{2} b_{3} b_{4}}$ is fixed by the inhomogeneous term only in the case in which the gluons with labels $b_{1}$ and $b_{2}$ are in a color singlet state. For the other irreducible representations we have no hint from the inhomogeneous term which would restrict the tensor $\Theta$. This problem seems to be a conceptual one in our approach. In the first step, that is for identifying a reggeizing part $D_{n}^{R}$ in the $n$-gluon amplitude, the quark loop was sufficient to fix the reggeizing part. Here it might be that the correct color tensor for the non-singlet states cannot be fixed unambiguously. Possibly the solution of this problem requires a better knowledge of the irreducible four-gluon amplitude $D_{4}^{I}$.

In spite of this conceptual problem it is well possible that one can find a simple ansatz that leads to further insight. In addition, we have the Ward type identities and the reggeization tensors derived in chapter 5 that can hopefully help us to find an ansatz of the kind (6.19) with a good choice of the tensors $\Theta$.

In section 5.2 we have found indications that a certain type of reggeized gluon has a special meaning in that is can occur in any amplitude, namely the reggeized gluon of type $f$ in the adjoint representation. It seems natural to make an ansatz in which this reggeized gluon plays an important role. To this end we split the amplitude $D_{6}^{I, R}$ we want to guess (see (6.19)) into three parts,

$$
D_{6}^{I, R}=D_{6}^{I, R 1}+D_{6}^{I, R 2}+D_{6}^{I, R 3} .
$$

The $f$-type reggeon can split into three gluons with a tensor $d^{a_{1} a_{2} a_{3} b}$. We therefore make the ansat

$$
D_{6}^{I, R 1}=g^{2} \sum d^{a_{1} a_{2} a_{3} c} D_{4}^{I c a_{4} a_{5} a_{8}}(123,4,5,6) .
$$

The sum extends over all possible groups of three gluons that are then merged into one reggeized gluon entering the irreducible four-gluon amplitude $D_{4}^{I}$. If we insert this into the integral equation (6.3) and apply the integral equation (3.4) for $D_{4}^{I}$ to the inserted term on the left hand side, those inhomogeneous terms on the right hand side are exactly cancelled which contain a function $V$ in which three momenta occur in a sum.

Since a $f$-type reggeon can also split into two gluons with a tensor $f_{a_{1} a_{2} c}$ we make the following ansatz for $D_{6}^{I, R 2}$ :

$$
D_{6}^{I, R 2}=-\frac{g^{2}}{4} \sum f_{a_{1} a_{2} c} f_{a_{3} a_{4} d} D_{4}^{I c d a_{5} a_{6}}(12,34,5,6) .
$$

The sum extends over all possible pairs of pairs out of the six gluons. The two gluons of each pair then merge into one reggeized gluon entering the amplitude $D_{4}^{I}$. Let us now see what happens when we insert this ansatz into the integral equation (6.3). We can then again apply the integral equation for the amplitude $D_{4}^{I}$ to the term inserted on the left hand side. This exactly cancels all terms on the right hand side contained in the functions $L$. The latter are thus completely eliminated by the ansatz $D_{6}^{I, R 2}$. In addition, a part of lhe inhome lat en an d $\delta_{a b}$ tensors. (There are two decompositions of this kind possible. We should choose the one 'matching' the momentum structures.) Then our ansatz $D_{6}^{l, K 2}$ cancels all those terms on the right hand side of the integral equation which contain a function $V$ that is multiplied with a tensor of the kind $f_{a_{1} a_{2} c} f_{c a_{3} a_{4}} \delta_{a_{5} a_{6}}$.

The cancellations mentioned above are of course accompanied by the emergence of other terms in the integral equation that contain convolutions of the function $D_{4}^{I}$ with kernels and products of $D^{I}$ with trajectory functions $\beta$. They will eventually contribute kernels and pr 政 is to four outgoing gluons.

But there is also a further class of possible terms in the equation which cannot contribute to such a vertex. In these terms a splitting of one gluon into two happens at the same moment in rapidity in which two other of the gluons interact via a kernel $K_{2 \rightarrow 3}$ to give three gluons. Such terms are contained in the last but one term in the integral equation (6.3) when the known solution (4.6) of the five gluon amplitude is inserted. The ansatz $D_{6}^{I, R 2}$ has the advantage of cancelling such terms by virtue of the mechanism (4.12) that brings about reggeization in a three-gluon subsystem. Such terms that contain a one-totwo transition and a two-to-three transition at the same moment in rapidity seem to be very difficult to be interpreted in the sense of a field theory. Therefore their cancellation is very appreciable in view of an effective field theory of unitarity corrections. We should mention that the ansatz $D_{6}^{I, R 1}$ does not produce such terms.

The choices $D_{6}^{I, R 1}, D_{6}^{I, R 2}$ of reggeizing parts seem to be very successful so far. Although the inhomogeneous term in the integral equation contains information only for the color singlet state of the two gluons that split (see discussion above) we have found a good ansatz in both cases inspired by the reggeization tensors derived in chapter 5 .

As we have already mentioned earlier the terms containing $d_{a b e}$ tensors should be treated differently. This becomes clear when we try to make an ansatz similar to $D_{6}^{I, R}$ with $d_{a b c}$ tensors instead of the $f_{a b c}$,

$$
-\frac{g^{2}}{4} \sum d_{a_{1} a_{2} c} d_{a_{3} a_{4} d} D_{4}^{I c d a_{5} a_{6}}(12,34,5,6)
$$


This ansatz produces too many $V$ 's in the integral equation. Each $D_{4}^{I}$ brings about three functions $V$ according to the integral equation (3.4). But the equation does not contain terms equivalent to the ones in the functions $L$ with (6.5). It seems that the two gluon can only split with $d$-type tensors if they are in a color singlet. But even with the help of a projection

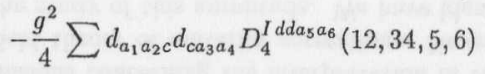

we would produce too many $V$ 's - this time with other color weights, but this does not help. A possible way out might be to define a new four-gluon amplitude $\tilde{D}_{4}^{I}$ by the integral equation

$$
\begin{aligned}
\left(\omega-\sum_{i=1}^{4} \beta\left(\mathrm{k}_{i}\right)\right) \tilde{D}_{4}^{I a_{1} a_{2} a_{3} a_{4}}\left(\mathbf{k}_{1}, \mathrm{k}_{2} ; \mathrm{k}_{3}, \mathrm{k}_{4}\right)= & \delta_{a_{1} a_{2}} \delta_{a_{3} a_{4}}\left(V D_{2}\right)\left(\mathrm{k}_{1}, \mathrm{k}_{2} ; \mathrm{k}_{3}, \mathrm{k}_{4}\right) \\
& +\sum K_{2 \rightarrow 2}^{\{b\} \rightarrow\{a\}} \otimes \tilde{D}_{4}^{I b_{1} b_{2} b_{3} b_{4}} .
\end{aligned}
$$

The full amplitude $D_{4}^{I}$ can then be obtained from the sum of three different $\tilde{D}_{4}^{I}$ 's. With the function $\tilde{D}_{4}^{I}$ instead of $D_{4}^{I}$ an ansatz of the kind (6.28) might work. A similar ansatz the function $D_{4}^{I}$ instead of $D_{4}^{I}$ an ansatz of the kind (6.28) might work. A similar ansatz
can then be formulated for the $\delta_{a b}$ tensor terms as well. With such an ansatz we will can then be formulated for the $\delta_{a b}$ tensor terms as well. With such an ansatz we will
be led to an equation for $D_{6}^{I, I}$ that contains the old two-to-four transition vertex acting on two of the gluons in $\tilde{D}_{4}^{I}$, but this is only true if the two gluons are in a color singlet state. However, there will be more terms. Especially, there will be terms that contain two-to-three transition at the same time with a one-to-two transition. This seems to us rather unattractive. In addition, there is an ambiguity in this ansatz since we actually do not know how to use the $d$-type tensors of chapter 5 in the color non-singlet channel.

It seems natural to ask whether the Ward type identities found in chapter 5 can provide help for finding a good ansatz of the kind (6.19). We will make an instructive observation here. Let us to this end assume for a moment that

$$
P^{R}=D_{6}^{I, R 1}+D_{6}^{I, R 2},
$$

is a reggeizing term, and let us see if it fulfills the Ward type identity. For this we se one momentum argument to zero. Due to the large number of terms in $(6.26),(6.27)$ the calculation is a bit tedious, and we only describe the outcome here. The result is that the ansatz $P^{R}$ does not fulfill the Ward type identity exactly. The outcome of

$$
\left.P^{R}\right|_{\mathrm{k}_{i}=0}
$$

differs from the one expected from the Ward type identity (see section 5.1 ) only by terms that do not contain $f_{a b c}$ tensors. Roughly speaking we can say that the Ward type identity is fulfilled modulo $d_{a b c}$ and $\delta_{a b}$ tensors. This is of course the best we can expect since these tensors have certainly not been taken into account correctly in $P^{R}$ (actually, they have been left out completely). This 'partial' applicability of the Ward type identity to $P^{R}$ gives us further confidence that the choices $(6.26),(6.27)$ are the right ones for a reggeizing part of the amplitude $D_{6}^{I}$. The prove of the 'partially' fulfilled Ward type identity heavily relies on the condition (2.54) for invariant tensors. This invariance condition contain contractions of an invariant tensor with $f$ ac tensors since they have a special meaning for the adjoint representation. A similar equation with $d_{a b c}$ tensors or even with $\delta_{a b}$ tensors does not exist. This certainly makes it technically very difficult to find an ansatz that fulfills the Ward type identities also for the $d_{a b c}$ and $\delta_{a b}$ tensors.

It was our intention in this section to give a survey of the results obtained so far in the second step of the analysis of the six-gluon amplitude. We have encountered technical as well as conceptual problems in finding a correct ansatz for the piece that reggeizes with respect to the irreducible four-gluon amplitude. Specifically, the problems concern the choice of the color structure of the ansatz. With the help of the results found in the previous chapter we have been able to find at least a part of a promising ansatz. We have outlined the problems related to the $\delta_{a b c}$ and $\delta_{a b}$ tensors. So far our analysis shows that the seemingly obvious choices for the part $D_{6}^{I, R 3}$ of the ansatz are unlikely to lead us to deeper insight on the field theory structure, and a more refined ansatz will be required.

\subsection{On a possible Pomeron-Odderon-Odderon vertex}

The Odderon is the $C=-1$ partner of the Pomeron, i. e. it carries negative charge parity. In perturbative $\mathrm{QCD}$ it consists of a compound state of three reggeized gluons described by the three-particle BKP equation $[18,20]$. The three gluons are in a completely symmetric state, and the color part of the wavefunction is a $d_{\text {a }}$ tensor. A natural question is whether aFKT Pomeron can be coupled to two Odders. The six. A nat am the triple Pomeron vertex arose from the two-to-four gluon transition vertex $V_{2 \rightarrow 4}$ when the latter was projected onto three BFKL eigenfunctions (see section 3.4). We can therefore in analogy try to project the inhomogeneous term of the new integral equation (6.3) onto two Odderon wavefunctions from below. The inhomogeneous term in that equation consists of several contributions, and we will concentrate here on the piece discussed in section 6.3. We therefore ask whether the integral

$$
\begin{gathered}
\int\left(\prod_{i=1}^{6} d^{2} \mathbf{k}_{i}\right)\left(W^{a_{1} a_{2} a_{3} a_{4} a_{5} a_{6}} D_{2}\right)\left(\mathbf{k}_{1}, \mathrm{k}_{2}, \mathrm{k}_{3}, \mathrm{k}_{4}, \mathrm{k}_{5}, \mathrm{k}_{6}\right) \\
\times d_{a_{1} a_{2} a_{3}} d_{a_{4} a_{5} a_{6}} \Psi_{1}\left(\mathrm{k}_{1}, \mathrm{k}_{2}, \mathrm{k}_{3}\right) \Psi_{2}\left(\mathrm{k}_{4}, \mathrm{k}_{5}, \mathrm{k}_{6}\right)
\end{gathered}
$$

is different from zero. Unfortunately, the wavefunction $\Psi$ of the Odderon is not known explicitly. But conformal invariance places strong constraints on the wavefunction. In [32] the wave function of the Odderon in impact parameter space was found to have the general form

$$
\Psi\left(\rho_{1}, \rho_{2}, \rho_{3}\right)=\left(\frac{\rho_{12} \rho_{13} \rho_{23}}{\rho_{10}^{2} \rho_{20}^{2} \rho_{30}^{2}}\right)^{\frac{h}{3}} \psi(x) .
$$

$h$ is the conformal weight of the Odderon state and $x$ is the anharmonic ratio

$$
x=\frac{\rho_{12} \rho_{30}}{\rho_{13} \rho_{20}}
$$

with

$$
\rho_{i j}=\rho_{i}-\rho_{j} .
$$

The wavefunction $\Psi$ vanishes when two of the coordinates of the three gluons in the Odderon coincide. This property of the Odderon drastically reduces the number of terms in $\left(W^{a_{1} a_{2} a_{3} a_{4} a_{5} a_{6}} D_{2}\right)$ that can give a non-vanishing contribution to the integral (6.33). If 
a term in $\left(W D_{2}\right)$ depends only on the sum of two momenta, say $k_{1}$ and $k_{2}$, then this term is after Fourier transformation to impact parameter space proportional to a delta-function of the two corresponding coordinates, i. e. proportional to $\delta\left(\rho_{1}-\rho_{2}\right)$. This implies a zero in the Odderon wavefunction and the corresponding term does not contribute to (6.33). Only very few standard integrals can actually give non-vanishing contributions. Some of them give identical results in the integral (6.33) due to the symmetry of the Odderon of them give identical results in the integral (6.33) due to the symmetry of the Odderon
wavefunction. (They are in this sense equivalent to each other as far as their contribution wavefunction. (They are in this sense equivalent to each other as far as their contribution
to (6.33) is concerned.) The possible contributions to the above integral can in this way be reduced to the following infrared finite combination of standard integrals:

$$
a(14,25)-\frac{1}{2} s(14,25)-\frac{1}{2} s(14,36) .
$$

We now have to look for these terms in the new piece $\left(W^{a_{1} a_{2} a_{3} a_{4} a_{5} a_{6}} D_{2}\right)$. Interestingly, the first of the permutations in $(6.11)$ - the color tensor of which exactly matches the color structure of the two Odderons - does not contain terms equivalent to the above combination. But the other permutations do contain such terms. For example, the term

$$
d_{a_{1} a_{2} a_{5}} d_{a_{3} a_{4} a_{6}}\left(W D_{2}\right)(1,2,5 ; 3,4,6)
$$

contains exactly the terms (6.37). The corresponding color contraction gives a factor

$$
d_{a_{1} a_{2} a_{5}} d_{a_{3} a_{4} a_{6}} d_{a_{1} a_{2} a_{3}} d_{a_{4} a_{5} a_{6}}=\left(\frac{N_{c}^{2}-4}{N_{c}}\right)^{2}\left(N_{c}^{2}-1\right) .
$$

We can therefore conclude that the new piece described in section 6.3 admits a PomeronOdderon-Odderon vertex. We do not study its properties any further here. It should probably be possible to write it in the form of a conformal three-point function as it was possible for the triple Pomeron vertex. We would like to mention that also some of the other inhomogeneous terms in the integral equation (6.3) give non-vanishing contributions when inserted in the integral (6.33) instead of $\left(W D_{2}\right)$.

In view of the presently somewhat unclear situation with the inhomogeneous terms in (6.3), especially with the new piece (see section 6.3 ), it seems too early for a conclusive interpretation of such a Pomeron-Odderon-Odderon vertex in the context of an effective field theory of unitarity correction.

\subsection{The six-gluon amplitude and the effective field theory}

In view of the problems and open questions mentioned in sections 6.3 and 6.4 it seems too early to draw final conclusions concerning the interpretation of the six-gluon amplitude in view of the effective field theory of unitarity corrections. Nevertheless, we have made substantial progress in the study of this amplitude. We have identified a reggeizing part $D^{R}$ that is a superposition of BFKL amplitudes. We have derived the integral equation for the remaining part. All terms occurring in this new equation have been analyzed, includin the new piece that was even found to be conformally invariant in impact parameter space. Unfortunately, its interpretation has not yet been clarified. We have then speculated on further reggeizing part of the six-gluon amplitude. Here we have encountered difficultie that have prevented us from solving the problem. But we have found further confirmatio for some of the conjectures which we have formulated in the previous chapter. Thereby we have made some progress in the understanding of the phenomenon of reggeization. Fin ally, we have found evidence for the existence of a possible Pomeron-Odderon-Odderon vertex but also here it seems too early for a conclusive interpretation.

The most important result of this chapter is contained in the integral equation for the remaining part $D_{6}^{I}$ of the six-gluon amplitude. The occurrence of the vertex function $V$ in this equation is an extremely strong indication for the fact that further reggeization with respect to the four-gluon compound state takes place in the six-gluon amplitude. Exactly this is the necessary prerequisite for the emergence of the field theory structure in the unitarity corrections. 


\section{Summary and Outlook}

The subject of this thesis was the Regge limit of Quantum Chromodynamics. As the physical process to consider we have chosen the scattering of highly virtual photons at very large energy. This process is from a theoretical point of view very clean since it can be treated within perturbation theory. In leading logarithmic approximation it is described by the BFKL Pomeron corresponding to the exchange of a bound state of two reggeized gluons in the $t$-channel. The BFKL Pomeron leads to a power-like growth of the total cross section. Thereby it eventually violates the Froissart-Martin bound which is derived from unitarity. According to this bound total hadronic cross sections cannot grow faster than logarithmic with energy. This problem renders the leading logarithmic approximation inconsistent. It necessitates the inclusion of subleading corrections to restore unitarity.

We have investigated unitarity corrections in the framework of the generalized leading logarithmic approximation. The unitarity corrections comprise contributions with a larger nu mber of reggeized gluons in the $t$-channel. As a framework for our investigations we have chosen the approach formulated by Bartels. In this approach a minimal set of subleading contributions is identified that leads to a unitary scattering amplitude, and unitarity is also restored in all subchannels. We do not make use of the large- $N_{c}$ approximation, i. e. the full structure of the gauge group is kept. The objects of study in this approach are $n$-gluon amplitudes obtained from the full scattering amplitude with the help of $t$-channel regreon unitarity relations. They are designed to describe the production of $n$ gluons in

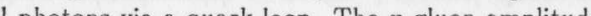
the $t$-channel

The known results on the three- and four-gluon amplitudes suggested that the unitarity corrections can be cast into an effective 2+1-dimensional conformal field theory. The two space-like coordinates correspond to impact parameter space, the time-like coordinat to rapidity. The first number-changing element of the effective field theory, the two-tofour gluon transition vertex, had been found in the analysis of the three- and four-gluon amplitudes. The aim of this thesis was to extend the knowledge about the effective fiel theory. The study of the five- and six-gluon amplitudes is the natural next step towards a better understanding of the unitarity corrections. The phenomenon of reggeization is very important for the emergence of the field theory structure. We have therefore also tried to gain more insight into the mechanism of reggeization. Especially the color structure of the $n$-gluon amplitudes was of interest in this respect.

In order to study the five- and six-gluon amplitudes it was first necessary to develop suitable methods that allow to treat the problem in spite of its complexity. The latter is mainly caused by the huge number of convolutions of amplitudes with integral kernels. We have used Birdtrack notation to accomplish the contractions in su $\left(N_{c}\right)$ algebra classification of the momentum space integrals occurring in the equations has been per- formed, and we have developed an algorithm for the implementation of the momentum space convolutions on a computer. The methods can be used for higher $n$-gluon amplitudes as well.

The results on the three- and four-gluon amplitudes have been reviewed in this thesis. The reggeization in these amplitudes was discussed. The general method used to tackle the integral equations has been explained with the example of the four-gluon amplitude We have described the properties of the two-to-four gluon transition vertex and of the irreducible four-gluon amplitude which are important also for the study of the higher $n$ gluon amplitudes. The field theory structure has been explained and we have emphasize its close connection with reggeization. The conformal invariance of the transition vertex has been reviewed. A new representation of the vertex was given in which the vertex is written as a sum of convolutions of BFKL kernels and free propagators only.

It was possible to solve the integral equation for the five-gluon amplitude. We have found the amplitude to be a superposition of two-gluon and four-gluon compound states. Stated differently, one part of the amplitude reggeizes with respect to the two-gluon state and the other part with respect to the irreducible four-gluon amplitude. This result generalizes the concept of reggeization to the higher level of the four-gluon compound state.

We then turned to the phenomenon of reggeization. It was observed that the reggeizing parts of the amplitudes with up to five gluons obey identities of a Ward type. These relate a given $n$-gluon amplitude in which one momentum argument is set to zero to the $(n-1)$ gluon amplitude. The Ward type identities were even shown to hold for generat $n$ for that formulated conjectures concerning the generalization of the Ward type identities to higher $n$-gluon amplitudes. The possible significance of the Ward type identities lies in the fact that they place strong constraints on the color structure of the amplitudes.

It was possible to study the reggeization of the gluon in the two-gluon compound state in very general form. The reggeized gluon represents an infinite sum of Feynman diagrams in which many gluons arrange to form a collective excitation of the gauge field. We have been able to find the color tensors accompanying this merging of gluons for all possible higher 'Fock states' of a reggeized gluon that is part of a BFKL amplitude This color che main information about the mechanism of reggeization in the twogluon amplitude. It was possible to assign a kind of quantum number to the reggeized gluon according to the way it is composite from gluons in color space. The possible quantum number assignments include two reggeized gluons in the adjoint representation. We have conjectured that one of these (the $f$-type reggeon, see section 5.2) acquires a special meaning. Namely, we expect it to occur also in more complicated amplitudes, like for instance in the irreducible four-gluon state, in the same manner as in the BFKL amplitude. For the other types we expect a more complicated behavior in other amplitudes. Evidence for this conjecture was found in the investigation of the six-gluon amplitude.

The six-gluon amplitude turned out to more difficult to understand than the lower $n$-gluon amplitudes. We applied our so far very successful method to split off a reggeizing part and derived a new integral equation for the remaining part. This step was the one in this thesis that required the greatest computational effort. The inhomogeneous term of the resulting integral equation consists of two pieces. One piece can be decomposed into functions that are known from the two-to-four vertex. This part clearly indicates that a. further reggeization takes place. Due to technical and conceptional difficulties we have 
not been able to gain a full understanding of this further reggeization. Nevertheless, we have found that it is possible to arrange all parts in such a way that a transition from two to six gluos can happen via the repeated transtion decribed by the known two-totour tranition four trangtion vertex. But this transition is uniguely deter steps. ? undergoing the transition are in a color singlet state in both steps. The generalization of the vertex to other color states causes serious problems. We have discussed some directions that might lead to a resolution of these problems. Another piece was found in the new integral equation that does not allow a decomposition in terms of the known two-to-fou vertex. We have discussed its properties in detail, including its conformal invariance. This piece might allow an interpretation as a new two-to-six transition vertex, i. e. as a new element in the effective field theory. However, a different interpretation seems viable as well. Therefore, a conclusive interpretation of this new piece has to be deferred

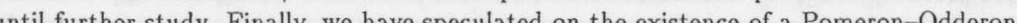
Dil firther stex. Fin Odderon vertex. In fact, we have found terms in the six gluon amplitude that do allow such a coupling. But a full understanding requires further investigation as well.

To summarize we can say that the amplitudes with up to five $t$-channel gluons are by now quite well understood. We have made considerable progress in the understanding of the phenomenon of reggeization. The first step towards finding the structure of the six-gluon amplitude has been performed. The result clearly indicates that a further reggeization takes place in the four-gluon compound state. This feeds the hope that the field theory structure will persist also to these more complicated elements of the unitarity corrections. The next step in the investigation of the six-ghon ampliture seems to require more refined methods. We have to leave their discovery for future research.

It is conceivable that the use of the integral equations is not sufficient to resolve the mentioned problems with the six-gluon amplitude. It would therefore be desirable to derive the effective field theory structure from a different starting point as well. Lipatov's ffective action approach might be best suited here. A different approach to the effective field theory would be very advisable also from a practical point of view. The computational effort necessary to investigate the $n$-gluon amplitudes increases very rapidly with $n$. The six-gluon amplitude might already be at the edge of being too complex to be treated with our method.

The study of the unitarity corrections with up to six reggeized gluons in the $t$-channel has shown that their structure is very many-faceted. The Regge limit of QCD is a longstanding and difficult problem and much hard work will still be required to unravel its mysteries.

\section{Appendix A}

\section{Color Algebra}

In this appendix we focus on more technical details of the color algebra that is a recurrent theme throughout this thesis. In section A.l we explain how the notation introduced in section 2.3 can be used to contract su $\left(N_{c}\right)$ tensors of arbitrary rank. We have applied the method for tensor contractions with up to six external gluon lines. Some of the basic results needed for the investigation of the $n$-gluon amplitudes are collected in section A.2.

\section{A.1 A method for contractions in $\operatorname{su}\left(N_{c}\right)$ algebra}

When doing the calculations sketched in chapters 3 through 6 a standard task would be to calculate contractions of the type

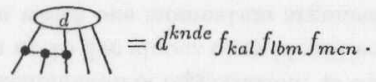

We will now outline an algorithm to solve problems of this kind. We restrict ourselves here to contractions where the outgoing lines correspond to gluon color representations. The method is, however, readily extended to arbitrary tensors involving quark representations as well. The following prescription can (and to avoid errors should) be carried out diagrammatically.

Let us call 'standard tensors' such tensors that are the sum or the difference of traces of generators of the form

$$
\operatorname{tr}\left(t^{a} \ldots t^{z}\right) \pm \operatorname{tr}\left(t^{z} \ldots t^{a}\right)
$$

like the ones defined in $(233),(2.34)$. The typical examples that occur in the analysis of the integral equations are $f_{a b c}, d_{a b c}, d^{a b c d}, f^{a b c d e}$, and $d^{a b c d e f}$. The first step is to express all standard tensors occurring in the diagram by their representation in term of generators, that is - diagrammatically speaking - by quark loops according to their respective definitions. (Here the terms 'quark line' and 'gluon line' refer to their respective color representation only.) For each of the standard tensors we then get two quark loops. The whole diagram is thus transferred to a sum of $2^{m}$ diagrams, $m$ being the number of Thendard tensors involved. Each of these diagrams contains only gluon lines and closed quark lines. It is natural to call all gluon lines starting on some closed quark line and ending on some closed quark line 'inner' gluon lines. 
The key ingredient for our method is the decomposition of a quark-antiquark state into a singlet and an adjoint representation mentioned already in section 2.3 ,

$$
\delta_{\gamma}^{\alpha} \delta_{\beta}^{\delta}=2\left(t^{a}\right)_{\beta}^{\alpha}\left(t^{a}\right)_{\gamma}^{\delta}+\frac{1}{N_{c}} \delta_{\beta}^{\alpha} \delta_{\gamma}^{\delta},
$$

$\alpha, \ldots, \delta$ being color labels in the fundamental representation. In birdtracks it is

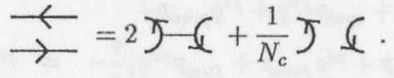

This is now in reversed order,

$$
J \in=\frac{1}{2} \longrightarrow-\frac{1}{2 N_{c}} \text { J }
$$

applied to all inner gluon lines. To do this properly one has to draw all quark loops in the diagrams counterclockwise before. This is not a mathematical operation although it might be quite some exercise in drawing. Applying (A.5) again considerably increases the number of diagrams, but the advantage is that we now can read off the result. The reason for this is the following. The use of (A.5) replaces each inner gluon line by two diagrams. In the first one, the inner gluon line is simply left out and does not connect the two closed quark lines ${ }^{1}$ any more. In the second one, the two quark loops are cut and joined to one closed quark line. Since we have applied (A.5) to all inner gluon lines, we are left with diagrams that only contain closed quark loops on which the outer gluon lines end. We can now join the diagrams back into rather compact expressions using the identity

$$
O=\operatorname{tr} 1=N_{c},
$$

the vanishing of the trace of $\mathrm{su}\left(N_{c}\right)$ generators

$$
\text { O- }=\operatorname{tr} t^{a}=0
$$

and the definitions of the standard tensors given in section 2.3. The latter are supplied by

$$
\begin{aligned}
& d_{a b c} d_{d e f}+f_{a b c} f_{d e f}=8\left[\operatorname{tr}\left(t^{a} t^{b} t^{c}\right) \operatorname{tr}\left(t^{f} t^{e} t^{d}\right)+\operatorname{tr}\left(t^{c} t^{b} t^{a}\right) \operatorname{tr}\left(t^{d} t^{e} t^{f}\right)\right] \\
& d_{a b c} d_{d e f}-f_{a b c} f_{d e f}=8\left[\operatorname{tr}\left(t^{a} t^{b} t^{c}\right) \operatorname{tr}\left(t^{d} t^{e} t^{f}\right)+\operatorname{tr}\left(t^{c} t^{b} t^{a}\right) \operatorname{tr}\left(t^{f} t^{e} t^{d}\right)\right]
\end{aligned}
$$

which is readily proved using the definition of the structure constants. In general, it is also necessary to use standard tensors of the type

$$
\begin{aligned}
f^{a b c d} & =-i\left[\operatorname{tr}\left(t^{a} t^{b} t^{c} t^{d}\right)-\operatorname{tr}\left(t^{d} t^{c} t^{b} t^{a}\right)\right] \\
d^{a b c d e} & =\operatorname{tr}\left(t^{a} t^{b} t^{c} t^{d} t^{e}\right)+\operatorname{tr}\left(t^{e} t^{d} t^{c} t^{b} t^{a}\right)
\end{aligned}
$$

- that is $f$-type tensors with an even number of color labels or $d$-type tensors with an odd nu mber of color labels - to the ones mentioned below equation (A.2). For the identities needed in this thesis (see next section) this is, however, not necessary.

In the case of the above example (A.1) the result of the procedure described here is

$$
\text { ता }=-\frac{N_{c}}{2} \text { ती }-\frac{1}{8} \cap \Lambda-\frac{1}{8} \wedge-\frac{1}{8} \wedge-\frac{1}{8} \wedge n \text {. }
$$

In many cases the above prescription can be shortened: for instance if a subdiagram can be reduced or equals zero, if the whole diagram can be obtained from some known diagram by permutation of outgoing gluon lines, or by using properties of the involved tensors like their invariance under cyclic permutations.

${ }^{1}$ They might be one and the same quark line, but that does not change our argument.

\section{A.2 Useful contractions of color tensors}

In this section we collect a series of $\mathrm{su}(N)$ identities $^{2}$ obtained with the help of the method explained in the previous section. The list does not exhaust the contractions needed for the calculations described in this thesis. Instead, we try to provide here a list of identities from which many others can be derived by a short or at least a simple calculation, for instance by permutations of the color labels or by combining different identities given here. In some cases, also the use of explicit decompositions of the different $d$ and $f$ tensors (see section 2.3) is useful. In particular, it should be possible to construct all contractions actually needed for the analysis of the $n$-gluon amplitudes up to $n=6$ discussed in this thesis without going through the whole program described in the preceding section. The definitions of all tensors used in this appendix and the normalization of $s u(N)$ generators can be found in section 2.3 .

For two external gluons we have

$$
\begin{aligned}
f_{l a k} f_{k b l} & =-N \delta_{a b} \\
d_{l a k} d_{k b l} & =\frac{N^{2}-4}{N} \delta_{a b} .
\end{aligned}
$$

For three external gluons the following identities hold:

$$
\begin{aligned}
& f_{k a l} f_{l b m} f_{m c k}=-\frac{N}{2} f_{a b c} \\
& d_{k a l} f_{l b m} f_{m c k}=-\frac{N}{2} d_{a b c} \\
& d_{k a l} l_{l b m} f_{m c k}=\frac{N^{2}-4}{2 N} f_{a b c} \\
& d_{k a l} d_{l b m} d_{m c k}=\frac{N^{2}-12}{2 N} d_{a b c} .
\end{aligned}
$$

The use of the last two identities can be avoided for the problems under consideration in this thesis. They have been added for the sake of completeness here. For considering the case of four external gluons the following identities are helpful:

$$
\begin{aligned}
f_{k a l} f_{l b m} f_{m c n} f_{n d k} & =N d^{a b c d}+\frac{1}{2}\left(\delta_{a b} \delta_{c d}+\delta_{a c} \delta_{b d}+\delta_{a d} \delta_{b c}\right) \\
d^{k l c d} f_{k a m} f_{m b l} & =-\frac{N}{2} d^{a b c d}-\frac{1}{4} \delta_{a b} \delta_{c d} \\
d^{k b l d} f_{k a m} f_{m c l} & =\frac{1}{4}\left(\delta_{a b} \delta_{c d}+\delta_{a d} \delta_{b c}\right)
\end{aligned}
$$

The following identities apply to five external gluons:

$$
\begin{aligned}
f_{\text {kal }} f_{l b m} f_{m c n} f_{\text {ndo }} f_{o e k}=N & f^{a b c d e} \\
+ & \frac{1}{4}\left(\delta_{a b} f_{c d e}+\delta_{a c} f_{b d e}+\delta_{a d} f_{b c e}+\delta_{a e} f_{b c d}\right. \\
& +f_{a d e} \delta_{b c}+f_{a c e} \delta_{b d}+f_{a c d} \delta_{b e}+f_{a b e} \delta_{c d} \\
& \left.+f_{a b d} \delta_{c e}+f_{a b c} \delta_{d e}\right)
\end{aligned}
$$
${ }^{2}$ To avoid possible confusion of the subscript $c$ in $N_{c}$ with a color label $c$ we omit the subscript and giv 


$$
\begin{aligned}
d^{k l d e} f_{k a m} f_{m b n} f_{n c l}= & -\frac{N}{2} f^{a b c d e} \\
& -\frac{1}{8}\left(\delta_{a b} f_{c d e}+\delta_{a c} f_{b d e}+f_{a d e} \delta_{b c}+f_{a b c} \delta_{d e}\right) \\
d^{k c l e} f_{k a m} f_{m b n} f_{n d l}= & \frac{1}{8}\left(\delta_{a c} f_{b d e}-\delta_{a e} f_{b c d}+f_{a d e} \delta_{b c}-f_{a c d} \delta_{b e}\right. \\
& \left.\quad+f_{a b e} \delta_{c d}+f_{a b c} \delta_{d e}\right) \\
f^{k l c d e} f_{k a m} f_{m b l}= & -\frac{N}{2} f^{a b c d e}-\frac{1}{8} \delta_{a b} f_{c d e} \\
f^{k b l d e} f_{k a m} f_{m c l}= & \frac{1}{8}\left(\delta_{a b} f_{c d e}+f_{a d e} \delta_{b c}-f_{a b c} \delta_{d e}\right)
\end{aligned}
$$

Six external gluons require even a few more of these identities:

$$
\begin{aligned}
f_{k a l} f_{l b m} f_{m c n} f_{n d o} f_{o e p} f_{p f k}=- & N d^{a b c d e f} \\
- & \frac{1}{2}\left(\delta_{a b} d^{c d e f}+\delta_{a c} d^{b d e f}+\delta_{a d} d^{b c e f}+\delta_{a e} d^{b c d f}\right. \\
& +\delta_{a f} d^{b c d e}+d^{a d e f} \delta_{b c}+d^{a c e f} \delta_{b d}+d^{a c d f} \delta_{b e} \\
& +d^{a c d e} \delta_{b f}+d^{a b e f} \delta_{c d}+d^{a b d f} \delta_{c e}+d^{a b d e} \delta_{c f} \\
& \left.+d^{a b c f} \delta_{d e}+d^{a b c e} \delta_{d f}+d^{a b c d} \delta_{e f}\right) \\
+ & \frac{1}{8}\left[\left(d_{a b c} d_{d e f}+f_{a b c} f_{d e f}\right)+\left(d_{a b d} d_{c e f}+f_{a b d} f_{c e f}\right)\right. \\
& +\left(d_{a b e} d_{c d f}+f_{a b e} f_{c d f}\right)+\left(d_{a b f} d_{c d e}+f_{a b f} f_{c d e}\right) \\
& +\left(d_{a c d} d_{b e f}+f_{a c d} f_{b e f}\right)+\left(d_{a c e} d_{b d f}+f_{a c c} f_{b d f}\right) \\
& +\left(d_{a c f} d_{b d e}+f_{a c f} f_{b d e}\right)+\left(d_{a d e} d_{b c f}+f_{a d e} f_{b c f}\right) \\
& +\left(d_{a d f} d_{b c e}+f_{a d f} f_{b c e}\right) \\
& \left.+\left(d_{a e f} d_{b c d}+f_{a e f} f_{b c d}\right)\right]
\end{aligned}
$$

$d^{k l e f} f_{k a m} f_{m b n} f_{n c o} f_{o d l}=\frac{N}{2} d^{a b c d e f}$

$+\frac{1}{4}\left(\delta_{a b} d^{c d e f}+\delta_{a c} d^{b d e f}+\delta_{a d} d^{b c e f}+d^{a d e f} \delta_{b c}\right.$

$$
\left.+d^{a c e f} \delta_{b d}+d^{a b e f} \delta_{c d}+d^{a b c d} \delta_{e f}\right)
$$

$-\frac{1}{16}\left[\left(d_{a b c} d_{d e f}+f_{a b c} f_{d e f}\right)+\left(d_{a e f} d_{b c d}+f_{a e f} f_{b c d}\right)\right.$

$+\left(d_{a c d} d_{b e f}+f_{a c d} f_{b e f}\right)$

$\left.+\left(d_{a b d} d_{c e f}+f_{a b d} f_{c e f}\right)\right]$

$d^{k d l f} f_{k a m} f_{m b n} f_{n c o} f_{o e l}=-\frac{1}{4}\left(\delta_{a d} d^{b c e f}+d^{a c e f} \delta_{b d}+d^{a b e f} \delta_{c d}+d^{a b c f} \delta_{d e}\right.$

$$
\left.+d^{a b c d} \delta_{e f}+\delta_{a f} c^{c b d e}+d^{b a d e} \delta_{c f}+d^{\text {cade }} \delta_{b f}\right)
$$

$+\frac{1}{16}\left[\left(d_{a b d} d_{c e f}+f_{a b d} f_{c e f}\right)+\left(d_{a c d} d_{b e f}+f_{a c d} f_{b e f}\right)\right.$

$+\left(d_{a e f} d_{b c d}+f_{a e f} f_{b c d}\right)+\left(d_{a d e} d_{b c f}-f_{a d e} f_{b c f}\right)$

$+\left(d_{a c f} d_{b d e}-f_{a c f} f_{b d e}\right)$

$\left.+\left(d_{a b f} d_{c d e}-f_{a b f} f_{c d e}\right)\right]$

$$
\begin{aligned}
f^{k l d e f} f_{k a m} f_{m b n} f_{n c l}= & \frac{N}{2} d^{a b c d e f}+\frac{1}{4}\left(\delta_{a b} d^{c d e f}+\delta_{a c} d^{b d e f}+d^{a d e f} \delta_{b c}\right) \\
& -\frac{1}{16}\left(d_{a b c} d_{d e f}+f_{a b c} f_{d e f}\right) \\
f^{k c l e f} f_{k a n} f_{m b n} f_{n d l}= & -\frac{1}{4}\left(\delta_{a c} d^{b d e f}+d^{a d e f} \delta_{b c}+d^{a b e f} \delta_{c d}+d^{b a c d} \delta_{e f}\right) \\
& +\frac{1}{16}\left[\left(d_{a b c} d_{d e f}+f_{a b c} f_{d e f}\right)+\left(d_{a c d}\left(d_{b e f}-f_{a c d} f_{b e f}\right)\right.\right. \\
& \left.\quad+\left(d_{a e f} d_{b c d}-f_{a e f} f_{b c d}\right)\right] \\
d^{k l c d e f} f_{k a m} f_{m b l}= & -\frac{N}{2} d^{a b c d e f}-\frac{1}{4} \delta_{a b} d^{\text {cdef }} \\
d^{k b l d e f} f_{k a m} f_{m c l}= & \frac{1}{4}\left(\delta_{a b} d^{\text {dedef }}+\delta_{b c} d^{\text {adef }}\right) \\
& -\frac{1}{16}\left(d_{a b c} d_{d e f}-f_{a b c} f_{d e f}\right) \\
d^{k b c l e f} f_{k a m} f_{m d l}= & -\frac{1}{4}\left(d^{a b c d} \delta_{e f}+\delta_{b c} d^{a d e f}\right) \\
& +\frac{1}{16}\left[\left(d_{a b c} d_{d e f}-f_{a b c} f_{d e f}\right)\right. \\
& \left.+\left(d_{a e f} d_{b c d}-f_{a e f} f_{b c d}\right)\right]
\end{aligned}
$$


We will in the following identify a set of momenta with the sum of its elements. With this identification the term in the amplitude $D_{l}^{R}$ we want to consider is

$$
D_{2}(\mathcal{A}, \mathcal{B})=D_{2}\left(\sum_{r=1}^{\# \mathcal{A}} \mathrm{q}_{j_{r}}, \sum_{s=1}^{\# \mathcal{B}} \mathrm{q}_{j_{s}}\right) .
$$

\section{Appendix B}

\section{A Combinatorial Method for the Momentum Space Integrals}

A main step in our investigation of a given $n$-gluon amplitude $D_{n}$ is to split it into reggeizing part $D_{n}^{R}$ and a remaining part. The reggeizing part is a superposition of BFKL amplitudes $D_{2}$. Starting from this ansatz a new integral equation for the remaining part of the amplitude is derived. In order to calculate its inhomogeneous term it is necessary to convolute the reggeizing parts $D_{l}^{R}$ of the $l$-gluon amplitudes (with $l \leq n$ ) with the integral kernels $K_{2 \rightarrow m}^{\{b\} \rightarrow\{a\}}(l+m-2=n)$ according to the original integral equations. The number of momentum space integrals emerging from these convolutions increases very rapidly with $n$. Therefore a method is required that allows to perform the momentum space convolutions with the help of a computer. In this appendix we present such an algorithm. It relies on the classification of possible momentum space integrals given in section 2.7 . With the help of our algorithm the momentum space integrals can be brought to their standard form. For obtaining a part of the results in this thesis we have implemented the algorithm in the PERL script language. After that a computer algebra program (like MAPLE for example) can be used to multiply the integrals with the corresponding color tensors and to finally collect all terms.

The main purpose of our method is to reduce the problem of convoluting amplitudes voly of volution of one specific term in the amplitude $D_{l}^{R}$ with the transition kernel $K_{2 \rightarrow m}^{\{b\} \rightarrow\{a\}}$ $(l+m-2=n)$, and only the momentum part of the kernel will be of interest in thi appendix. The method can then successively be applied to all possible convolutions of individual terms in the reggeizing parts of the amplitudes with the integral kernels.

Let us now consider one specific term in the reggeizing part $D_{l}^{R}$ of the $l$-gluon amplitude. It is given by a BFKL amplitude that has two momentum arguments. Each of them is the sum of a subset of the $l$ momenta $q_{j}$. Let us call these two subsets $\mathcal{A}$ and $\mathcal{B}$, respectively. Their union exhausts the $l$ momenta,

$$
\mathcal{A} \cup B=\left\{\mathrm{q}_{1}, \ldots, \mathrm{q}_{l}\right\},
$$

and each of them contains at least one element,

$$
1 \leq \# \mathcal{A}, \# B \leq l-1 \text {. }
$$

Now we want to convolute this term with an integral kernel. Only two of the $l$ momenta $\mathrm{q}_{j}$ will actually be affected by the convolution. Let us call these two momenta $\mathrm{v}$ and $\mathbf{w}$,

$$
\mathbf{v}, \mathbf{w} \in\left\{\mathrm{q}_{1}, \ldots, \mathrm{q}_{l}\right\} ; \mathbf{v} \neq \mathbf{w} .
$$

The kernel $K_{2 \rightarrow m}^{\{b\} \rightarrow\{a\}}$ was given explicitly in section 2.6. We neglect the coupling constant $g$ and the color tensor for the purpose of this appendix. The momentum part of the kernel is according to (2.70)

$$
\begin{aligned}
& K_{2 \rightarrow m}\left(\mathbf{v}, \mathrm{w} ; \mathrm{k}_{i_{1}}, \ldots, \mathrm{k}_{i_{m}}\right)=\left(\mathrm{k}_{i_{1}}+\ldots+\mathrm{k}_{i_{m}}\right)^{2}-\frac{\mathrm{w}^{2}\left(\mathrm{k}_{i_{1}}+\ldots+\mathrm{k}_{i_{m-1}}\right)^{2}}{\left(\mathrm{k}_{i_{m}}-\mathrm{w}\right)^{2}} \\
& -\frac{\mathrm{v}^{2}\left(\mathrm{k}_{i_{2}}+\ldots+\mathrm{k}_{i_{m}}\right)^{2}}{\left(\mathrm{k}_{i_{1}}-\mathrm{v}\right)^{2}}+\frac{\mathrm{v}^{2} \mathrm{w}^{2}\left(\mathrm{k}_{i_{2}}+\ldots+\mathrm{k}_{i_{m-1}}\right)^{2}}{\left(\mathrm{k}_{i_{1}}-\mathrm{v}\right)^{2}\left(\mathrm{k}_{i_{m}}-\mathrm{w}\right)^{2}}
\end{aligned}
$$

The last term is not present if $m=2$. The momenta $\mathbf{k}_{i_{i}}$ with $(t \in\{1, \ldots, m\})$ are $m$ of the $n$ momenta that occur in the integral equation for $D_{n}$. Due to the condition that $t$-channel gluons do not cross in the integral equations (see section 2.2) they are ordered:

$$
1 \leq i_{1}<\ldots<i_{m} \leq n .
$$

Which $m$ of the $n$ gluons in the integral equation enter the kernel from below depends of course on the term we have chosen in the sums on the right hand side of the integral equations (2.11)-(2.15). The quantity we want to calculate here is the convolution

$$
K_{2 \rightarrow m}\left(\mathbf{v}, \mathbf{w} ; \mathrm{k}_{i_{1}}, \ldots, \mathbf{k}_{i_{m}}\right) \otimes D_{2}(\mathcal{A}, \mathcal{B}),
$$

where the symbol $\otimes$ again includes an integral over the loop momentum and the two propagators $\frac{1}{v^{2}} \frac{1}{w^{2}}$. The kernel acts trivially on the other $l-2$ momenta in the term $D_{2}(A, B)$. Our algorithm will leave them unchanged, that is after its application we are still left with some $\mathrm{q}_{j}$ 's in the standard integral. They have to be replaced in the end by the respective $\mathbf{k}_{i}$ 's. Mathematically speaking this is done by the one-to-one map

$$
\mathcal{A} \cup \mathcal{B} \backslash\{\mathrm{v}, \mathrm{w}\} \longrightarrow\left\{\mathrm{k}_{1}, \ldots, \mathrm{k}_{n}\right\} \backslash\left\{\mathrm{k}_{i_{1}}, \ldots, \mathrm{k}_{i_{m}}\right\}
$$

which has to be applied in ascending order according to the occurrence of the moment on both sides. On the left had side we have the $l-2$ momenta in $D_{2}(\mathcal{A}, \mathcal{B})$ not affected by the kernel, on the right hand side we find the $n-m=l-2$ momenta that are not attached to the kernel from below.

In addition, one more step has to be performed to finish the result after the rules below have been applied. This is connected with the definition of the second argument of the function a which was one of our standard integrals. As described in section 27 the

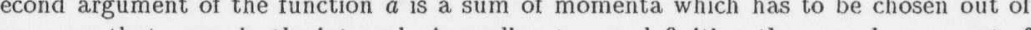


$a$ is the group of momenta that contains the momentum $\mathrm{k}$ with the lowest index. In our general treatment in this appendix it is not convenient to implement this condition from the beginning. Instead we adjust the resulting standard integrals in the end. This is done very easily. For example, if we have $n=4$ and the algorithm below leads to the result $a(2,34)$ then this should be replaced by $a(2,1)$.

We will treat the four parts of the kernel in (B.5) separately now. The resulting standard integrals have to be added in the end.

\section{First part of the kernel}

The first term in the kernel (B.5) is

$$
P\left(\mathbf{v}, \mathbf{w} ; \mathrm{k}_{i_{1}}, \ldots, \mathbf{k}_{i_{m}}\right)=\left(\mathrm{k}_{i_{1}}+\ldots+\mathrm{k}_{i_{m}}\right)^{2},
$$

and we want to bring the convolution

$$
P\left(\mathrm{v}, \mathbf{w} ; \mathbf{k}_{i_{1}}, \ldots, \mathbf{k}_{i_{m}}\right) \otimes D_{2}(\mathcal{A}, \mathcal{B})
$$

to its standard form. Let the set $\mathcal{X}$ be

$$
\mathcal{X}=\left\{\mathrm{k}_{i_{1}}, \ldots, \mathrm{k}_{i_{m}}\right\} .
$$

Then the different possible cases are

1. $\mathrm{v}$ and $\mathrm{w}$ are elements of the same set $\mathcal{A}$ or $\mathcal{B}$.

We then denote this set $(\mathcal{A}$ or $\mathcal{B})$ by $\mathcal{C}$.

(a) $\# \mathcal{C}=2$

The integral is $t(\mathcal{X})$.

(b) $\# \mathcal{C}>2$

The integral is $s(\mathcal{X}, \mathcal{C} \backslash\{\mathrm{v}, \mathrm{w}\})$

2. $\mathrm{v}$ and $\mathrm{w}$ are not elements of the same set $\mathcal{A}$ or $B$.

(a) $(\# \mathcal{A}=1) \wedge(\# \mathcal{B}=1)$

The integral is $c(\mathcal{X})$.

(b) $(\# \mathcal{A}=1) \wedge(\# \mathcal{B}>1)$

The integral is $b(\mathcal{X})$.

(c) $(\# \mathcal{A}>1) \wedge(\# \mathcal{B}=1)$

The integral is $b(\mathcal{X})$.

(d) $(\# \mathcal{A}>1) \wedge(\# \mathcal{B}>1)$

The integral is $a(\mathcal{X}, \mathcal{A} \backslash\{\mathrm{v}, \mathrm{w}\})$

Second part of the kernel

The second term in the kernel (B.5) is

$$
Q\left(\mathrm{v}, \mathrm{w} ; \mathrm{k}_{i_{1}}, \ldots, \mathrm{k}_{i_{m}}\right)=\frac{\mathrm{w}^{2}\left(\mathrm{k}_{i_{1}}+\ldots+\mathrm{k}_{i_{m-1}}\right)^{2}}{\left(\mathrm{k}_{i_{m}}-\mathrm{w}\right)^{2}},
$$

and we want to bring the convolution

$$
Q\left(\mathrm{v}, \mathbf{w} ; \mathbf{k}_{i_{1}}, \ldots, \mathbf{k}_{i_{m}}\right) \otimes D_{2}(\mathcal{A}, B)
$$

to its standard form. Let now the set $\mathcal{X}$ denote

$$
\mathcal{X}=\left\{\mathrm{k}_{i_{1}}, \ldots, \mathrm{k}_{i_{m-1}}\right\},
$$

and let the set $\mathcal{Y}$ be

$$
\mathcal{y}=\left\{\mathbf{k}_{i_{m}}\right\} .
$$

Then the different possible cases are

1. $\mathrm{v}$ and $\mathrm{w}$ are elements of the same set $\mathcal{A}$ or $\mathcal{B}$.

We then denote this set $(A$ or $B)$ by $C$.

The integral is $s(\mathcal{X},(\mathcal{C} \backslash\{\mathrm{v}, \mathrm{w}\}) \cup \mathcal{Y})$.

2. $v$ and $w$ are not elements of the same set $\mathcal{A}$ or $B$.

Let the set $(\mathcal{A}$ or $\mathcal{B})$ containing $\mathrm{v}$ be $\mathcal{C}$.

$$
\begin{aligned}
& \text { (a) } \# C=1 \\
& \text { The integral is } b(\mathcal{X}) . \\
& \text { (b) } \# \mathcal{C}>1 \\
& \text { The integral is } a(\mathcal{X}, \mathcal{C} \backslash\{\mathbf{v}\}) .
\end{aligned}
$$

\section{Third part of the kernel}

The third term in the kernel (B.5) is

$$
R\left(\mathrm{v}, \mathrm{w} ; \mathrm{k}_{i_{1}}, \ldots, \mathrm{k}_{i_{m}}\right)=\frac{\mathbf{v}^{2}\left(\mathrm{k}_{i_{2}}+\ldots+\mathrm{k}_{i_{m}}\right)^{2}}{\left(\mathrm{k}_{i_{1}}-\mathrm{v}\right)^{2}},
$$

and we want to bring the convolution

$$
R\left(\mathbf{v}, \mathbf{w} ; \mathbf{k}_{i_{1}}, \ldots, \mathbf{k}_{i_{m}}\right) \otimes D_{2}(\mathcal{A}, \mathcal{B})
$$

to its standard form. Let now the set $\mathcal{X}$ denote

$$
\mathcal{X}=\left\{\mathbf{k}_{i_{2}}, \ldots, \mathbf{k}_{i_{m}}\right\},
$$

and let now the set $y$ be

$$
y=\left\{k_{i_{1}}\right\} .
$$

Then the different possible cases are

1. $\mathrm{v}$ and $\mathrm{w}$ are elements of the same set $\mathcal{A}$ or $\mathcal{B}$.

We then denote this set $(\mathcal{A}$ or $\mathcal{B})$ by $\mathcal{C}$.

The integral is $s(\mathcal{X},(\mathcal{C} \backslash\{\mathrm{v}, \mathrm{w}\}) \cup \mathcal{Y})$.

2. $\mathrm{v}$ and $\mathrm{w}$ are not elements of the same set $\mathcal{A}$ or $B$. Let the set $(\mathcal{A}$ or $\mathcal{B})$ containing w be $\mathcal{C}$.
(a) $\# \mathcal{C}=1$
The integral is $b(\mathcal{X})$
(b) $\# \mathcal{C}>1$
The integral is $a(\mathcal{X}, C \backslash\{\mathbf{w}\})$. 
Fourth part of the kernel

The fourth term in the kernel (B.5) is

$$
S\left(\mathrm{v}, \mathrm{w} ; \mathrm{k}_{i_{1}}, \ldots, \mathrm{k}_{i_{m}}\right)=\frac{\mathbf{v}^{2} \mathrm{w}^{2}\left(\mathbf{k}_{i_{2}}+\ldots+\mathrm{k}_{i_{m-1}}\right)^{2}}{\left(\mathrm{k}_{i_{1}}-\mathbf{v}\right)^{2}\left(\mathrm{k}_{i_{m}}-\mathrm{w}\right)^{2}},
$$

and we want to bring the convolution

$$
S\left(\mathrm{v}, \mathrm{w} ; \mathrm{k}_{i_{1}}, \ldots, \mathrm{k}_{i_{m}}\right) \otimes D_{2}(\mathcal{A}, \mathcal{B})
$$

to its standard form. Let now the set $\mathcal{X}$ denote

$$
\mathcal{X}=\left\{\mathrm{k}_{i_{2}}, \ldots, \mathrm{k}_{i_{m-1}}\right\},
$$

and let now the set $y$ be

$$
y=\left\{k_{i_{1}}\right\}
$$

Let in addition the set $\mathcal{Z}$ be

$$
\mathcal{Z}=\left\{\mathrm{k}_{i_{m}}\right\}
$$

Then the different possible cases are

1. $\mathrm{v}$ and $\mathrm{w}$ are elements of the same set $\mathcal{A}$ or $B$. We then denote this set $(\mathcal{A}$ or $\mathcal{B})$ by $\mathcal{C}$.

The integral is $s(\mathcal{X},(\mathcal{C} \backslash\{\mathrm{v}, \mathrm{w}\}) \cup \mathcal{Y} \cup \mathcal{Z})$

2. $\mathrm{v}$ and $\mathrm{w}$ are not elements of the same set $\mathcal{A}$ or $\mathcal{B}$.

Let the set $(\mathcal{A}$ or $\mathcal{B})$ containing $\mathrm{v}$ be $\mathcal{C}$.

The integral is $a(\mathcal{X},(\mathcal{C} \backslash\{\mathbf{v}\}) \cup \mathcal{Y})$

\section{Bibliography}

[1] P.D. B. Collins, An Introduction to Regge Theory and High Energy Physics, Cambridge University Press, Cambridge 1977

[2] V.N. Gribov, Sov. Phys. JETP 26(2) (1968), 414

[3] M. Baker, K. A. Ter-Martirosyan, Phys. Rept. 28 C (1976), 1

[4] A. Donnachie, P. V. Landshoff, Phys. Lett. B 296 (1992), 227

[5] S.J. Brodsky, F. Hautmann, D. E. Soper, Phys. Rev. Lett. 78 (1997) 803, erratum ibid. 79 (1997) 3522 ;

S.J. Brodsky, F. Hautmann, D.E. Soper, Phys. Rev. D 56 (1997), 6957

[6] J. Bartels, A. De Roeck, H. Lotter, Phys. Lett. B 389 (1996), 742

J. Bartels, A. De Roeck, C. Ewerz, H. Lotter, to appear in "ECFA/DESY Study on Physics and Detectors for a Linear Collider", ed. R. Settles, DESY 97-123E (to be published), hep-ph/9710500

[7] A.H. Mueller, Nucl. Phys. Proc. Suppl. 18 C (1991), 125

[8] J. Bartels, A. De Roeck, M. Loewe, Z. Phys. C 54 (1992), 635 J. Kwieciński, A.D. Martin, P.J. Sutton, Phys. Lett. B 287 (1992), 254; J. Kwieciński, A.D. Martin, P.J. Sutton, Phys. Rev. D 46 (1992), 921;

W.-K. Tang, Phys. Lett. B 278 (1992), 363;

J. Bartels, M. Besancon, A. De Roeck, J. Kurzhoefer, in Proceedings of the Workshop on Physics at HERA, Hamburg 1991, eds. W. Buchmüller and G. Ingelman, p. 203

[9] J. Bartels, V. Del Duca, A. De Reock, D. Graudenz, M. Wüsthoff, Phys. Lett. B 384 (1996), 300

[10] E. A. Kuraev, L. N. Lipatov, V.S. Fadin, Sov. Phys. JETP 45 (1977), 199

[11] Ya. Ya. Balitskii, L. N. Lipatov, Sov. J. Nucl. Phys. 28 (1978), 822

[12] L. N. Lipatov, Sov. J. Nucl. Phys. 23 (1976), 338

[13] L. N. Lipatov, Sov. Phys. JETP 63(5) (1986), 904

[14] M. Froissart, Phys. Rev. 123 (1961), 1053

[15] A. Martin, Phys. Rev. 129 (1963), 1432 
[16] L. V. Gribov, E.M. Levin, M. G. Ryskin, Phys. Rept. 100 (1983), 1

[17] J. Bartels, Nucl. Phys. B 151 (1979), 293

[18] J. Bartels, Nucl. Phys. B 175 (1980), 365

[19] J. Bartels, DESY 91-074 (unpublished)

[20] J. Kwieciński, M. Praszalowicz, Phys. Lett. B 94 (1980), 413

[21] J. Bartels, Phys. Lett. B 298 (1993), 204;

J. Bartels, Z. Phys. C 60 (1993), 471

[22] J. Bartels, M. Wüsthoff, Z. Phys. C 66 (1995), 157

[23] J. Bartels, L. N. Lipatov, M. Wüsthoff, Nucl. Phys. B 464 (1996), 298

[24] H. Lotter, PhD Thesis, Hamburg University 1996, DESY 96-262, hep-ph/9705288

[25] V. S. Fadin, L. N. Lipatov, DESY-98-033, hep-ph/9802290 and references therein

[26] M. Ciafaloni, G. Camici, DFF-302-03-98, hep-ph/9803389 and references therein

[27] D. A. Ross, SHEP-98-06, hep-ph/9804332

[28] Yu. V. Kovchegov, A. H. Mueller, CU-TP-889, hep-ph/9805208

[29] L. N. Lipatov, JETP Lett. 59 (1994), 596

[30] L. D. Faddeev, G. P. Korchemsky, Phys. Lett. B 342 (1995), 311

[31] G.P. Korchemsky, Nucl. Phys. B 443 (1995), 255; G.P. Korchemsky, Nucl. Phys. B 462 (1996), 333;

G.P. Korchemsky, I. M. Krichever, Nucl. Phys. B 505 (1997), 387

[32] L. N. Lipatov, Phys. Lett. B 251 (1990), 284

[33] R. A. Janik, J. Wosiek, TPJU-2-98, hep-th/9802100

[34] A.H. Mueller, Nucl. Phys. B 415 (1994), 373

[35] H. Navelet, S. Wallon, SACLAY-SPHT-97-023, hep-ph/9705296

[36] A. H. Mueller, B. Patel, Nucl. Phys. B 425 (1994), 47; A. H. Mueller, Nucl. Phys. B 437 (1995), 107;

Zhang Chen, A.H. Mueller, Nucl. Phys. B 451 (1995), 579;

Yu. V. Kovchegov, A. H. Mueller, S. Wallon, Nucl. Phys. B 507 (1997), 367

[37] G.P. Salam, Nucl. Phys. B 461 (1996), 512;

A. H. Mueller, G.P. Salam, Nucl. Phys. B 475 (1996), 293

G.P. Salam, Comput. Phys. Comm. 105 (1997), 62

[38] R. Peschanski, Phys. Lett. B 409 (1997), 491
[39] L. N. Lipatov, Nucl. Phys. B 365 (1991), 614;

R. Kirschner, L. N. Lipatov, L. Szymanowski, Nucl. Phys. B 425 (1994), 579; R. Kirschner, L. N. Lipatov, L. Szymanowski, Phys. Rev. D 51 (1995), 838;

L. N. Lipatov, Nucl. Phys. B 452 (1995), 369

[40] L.N. Lipatov, Phys. Rept. 286 (1997), 131

[41] H. Verlinde, E. Verlinde, PUPT-1319, hep-th/9302104

[42] I. Balitsky, Nucl. Phys. B 463 (1996), 99

[43] O. Nachtmann, Ann. Phys. 209 (1991), 436

[44] H. G. Dosch, Phys. Lett. B 190 (1987), 177;

H. G. Dosch, Y. A. Simonov, Phys. Lett. B 205 (1988), 339;

Y. A. Simonov, Nucl. Phys. B 307 (1988), 512

[45] W. Buchmüller, A. Hebecker, Nucl. Phys. B 476 (1996), 203;

W. Buchmüller, M.F. McDermott, A. Hebecker, Nucl. Phys. B 487 (1997), 283, erratum ibid. B $\mathbf{5 0 0}$ (1997), 621

[46] J. R. Forshaw, D. A. Ross, Quantum Chromodynamics and the Pomeron, Cambridge University Press, Cambridge 1997

[47] L. N. Lipatov, in Perturbative Quantum Chromodynamics, ed. A. H. Mueller, World Scientific, Singapore, 1989

[48] J. Bartels, H. Lotter, Phys. Lett. B 309 (1993), 400;

J. Bartels, H. Lotter, M. Vogt, Phys. Lett. B 373 (1996), 215

[49] A. H. Mueller, Eur. Phys. J. A 1 (1998), 19

[50] V.N. Gribov, L. N. Lipatov, Sov. J. Nucl. Phys. 15 (1972), 438; Yu. L. Dokshitzer, Sov. Phys. JETP 46 (1977), 641;

G. Altarelli, G. Parisi, Nucl. Phys. B 126 (1977), 298

[51] H.D.I. Abarbanel, J.D. Bronzan, R.L. Sugar, A.R. White, Phys. Rept. 21 C (1975), 119

[52] A. R. White, ANL-HEP-PR-97-95, hep-ph/9712466

[53] R. C. Brower, C.E. DeTar, J. H. Weis, Phys. Rept. 14 C (1974), 259

[54] A. R. White, Int. J. Mod. Phys. A 8 (1993), 4755

[55] P. Cvitanovic, Group Theory, part I, Classics lllustrated, Nordita notes, Jan. 1984

[56] E. M. Levin, M. G. Ryskin, Sov. J. Nucl. Phys. 53 (1991), 653

[57] M. Braun, G.P. Vacca, hep-ph/9711486

[58] G.P. Vacca, PhD Thesis, University of Bologna, hep-ph/9803283

[59] J. Bartels, L. N. Lipatov, H. Lotter, in preparation 
[60] A. A. Belavin, A. M. Polyakov, A. B. Zamolodchikov, Nucl. Phys. B 241 (1984), 333

[61] P. Ginsparg in Les Houches, Session XLIX, 1988, eds. E. Brézin and J. Zinn-Justin (Elsevier Science Publishers B.V., 1989)

[62] G.P. Korchemsky, LPTHE-ORSAY-97-62, hep-ph/9711277

[63] A. Bialas, H. Navelet, R. Peschanski, SACLAY-T97-131, hep-ph/9711236

[64] M. Braun, SPBU-97-9, hep-ph/9706373

[65] J. Bartels, private communication

\section{Acknowledgements}

First of all I am very grateful to my supervisor Prof. J. Bartels for many interesting discussions, for support and for his constant interest in my work.

I consider myself very lucky to have known the late Prof. V.N. Gribov. In many discussions with him I benefitted from his deep understanding of physics. The conversations with him were always very pleasant and motivating, and his interest in my work was very encouraging for me. It is very sad that he passed away much too early.

I would like to express my gratitude to Hans Lotter. I enjoyed countless discussions with him on physics and non-physics issues. He never failed to willingly answer all my questions about QCD which I very often asked in late-night phone calls.

Many thanks go to Mark Wüsthoff for interesting conversations which were very helpful for my understanding of $\mathrm{QCD}$ in the Regge limit.

I am grateful to Gregory Korchemsky and Prof. L.N. Lipatov for very instructive and enjoyable discussions on high energy QCD. I am also grateful to Prof. H. Joos for many discussions which helped me to look at my work in a wider context.

For a careful reading of large parts of the manuscript I am grateful to Hans Lotter, Ulrike Wollmer, and Mark Wüsthoff. Their comments helped to improve this thesis substantially. They are in no way responsible for the errors that have escaped their critical eyes.

This work was in part supported by the Deutsche Forschungsgemeinschaft and by the Bundesministerium für Bildung, Wissenschaft, Forschung und Technologie.

I would like to thank all my friends and colleagues at the II. Institut für Theoretische Physik and DESY for creating a very pleasant working atmosphere. Special thanks go to my friend Oskar for nice distraction.

Finally, I would like to express my gratitude to my parents and Zotty for constant support and encouragement during all the years. 
